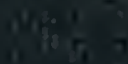

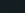





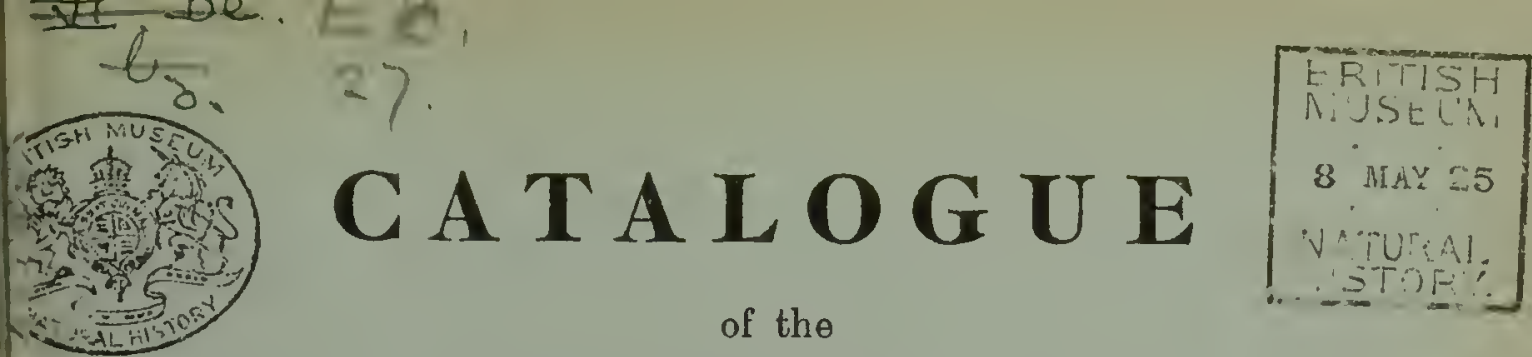

\title{
Type Specimens
}

of

\section{Lepidoptera Rhopalocera}

in the

\section{BRITISH MUSEUM}

\author{
Part II. DANAIDAE
}

BY

N. D. RILEY

Assistant in the Department of Entomology

AND

A. G. GABRIEL

LONDON :

PRINTED BY ORDER OF THE TRUSTEES

OF THE BRITISH MUSEUM.

Sold at the British Museub (Natural History), Cromwell Road, S. W. 7 ;

AND BY

B. Quaritch, Ltd. ; Dulau \& Co., Ltd.;

The Oxford University Press; Wheldon \& Wesley, Ltd., London;

and OLIVEr and Boyd, EDinburah.

$$
1925 .
$$

Price Four Shillings and Sixpence 



\title{
CA T A L O G U E \\ of the
}

\section{Type Specimens}

of

\section{Lepidoptera Rhopalocera} in the

\section{BRITISH MUSEUM}

\author{
PART II. DANAIDAE
}

BY

\author{
N. D. RILEY
}

Assistant in the Department of Entomology

AND

A. G. GABRIEL

\section{LONDON : \\ PRINTED BY ORDER OF THE TRUSTEES \\ OF THE BRITISH MUSEUM.}

Sold at the Brisish Museem (Natural History), Cromwell Road, S.W. 7 ; AND BY

B. Quaritch, Ltd.; Dulau \& Co., Ltd.;

The Oxford University Press; Wheldon \& Weslex, Ltd., London;

and Oliver and Boyd, Edinburgi.

1925.

Ali rights reserved 


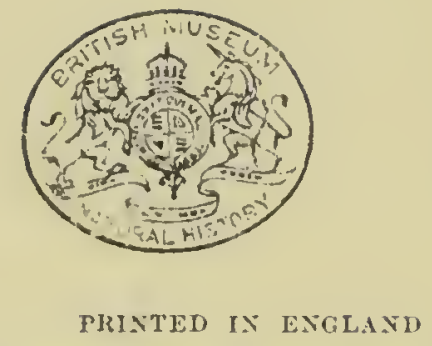

A'T THE OXFORD UNIVERSTTY PRESS

BL FREDERICK HATL 


\section{PREFACE TO PART II}

Since Part I of this Catalogue was issued, some 1,200 Type specimens belonging to the family Danaidae have been listed, and the results are embodied in this Part.

The only departurc from the practice adopted in Part I has been the inclusion of Type localities. These, it will be found, do not always agree with the published localities. In cases of disagrcement the localities now given are the more accurate, and sliould be accepted.

British Museum

C. J. Gahan, (Natural History), Keeper of Entomology. 16th March, 19:5. 



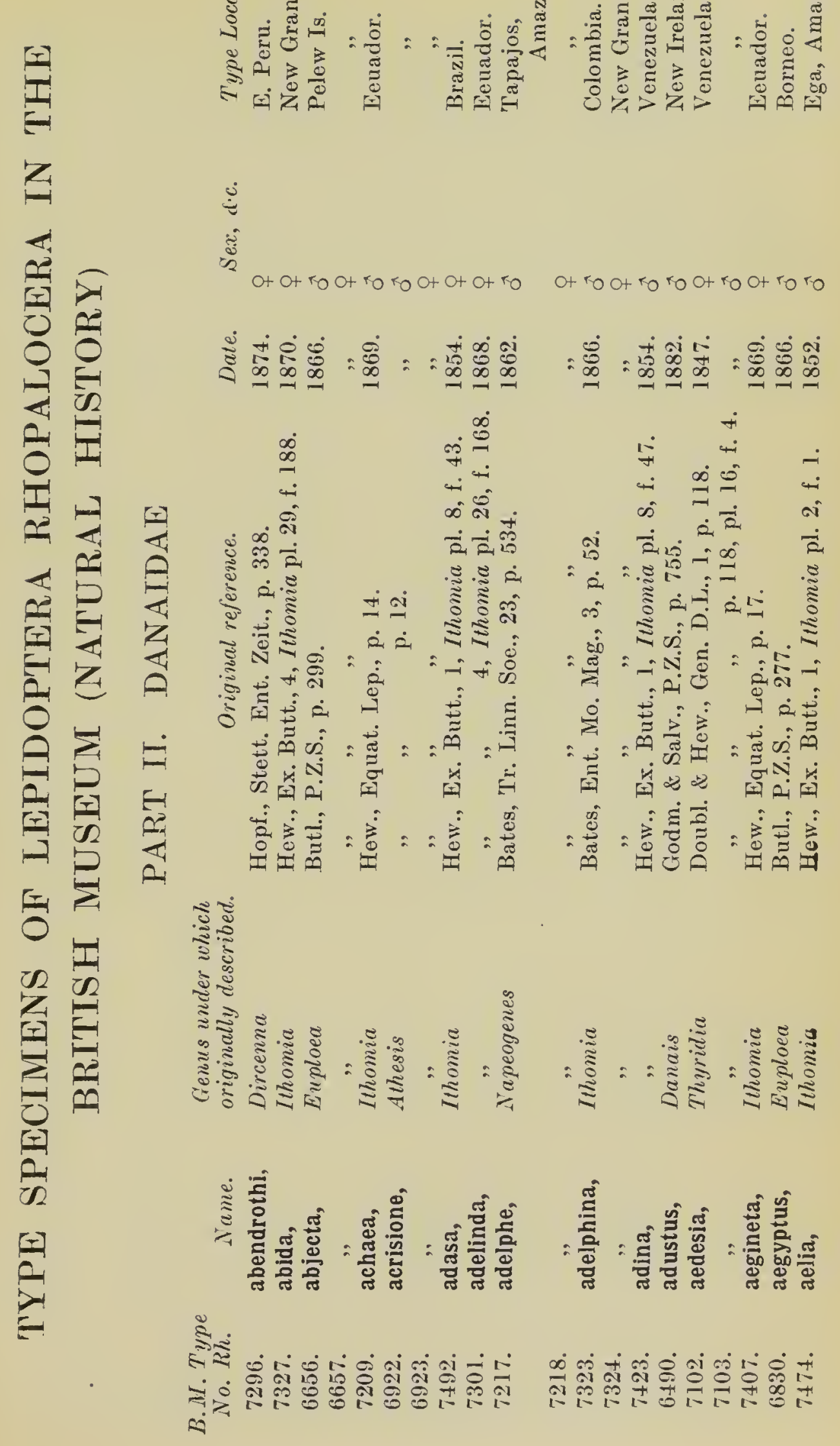




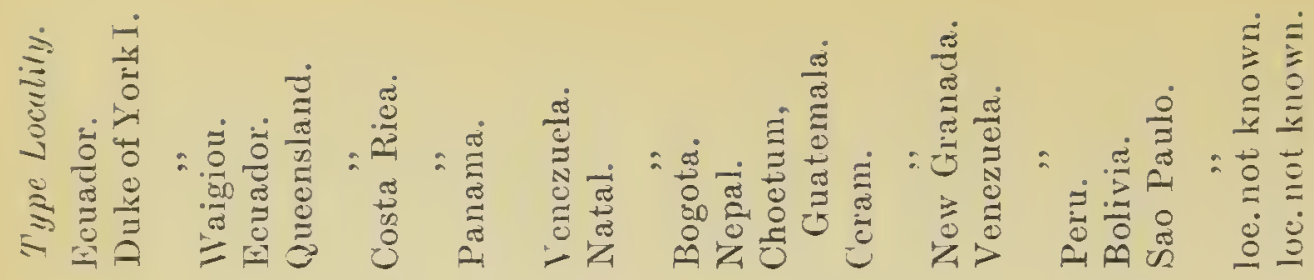

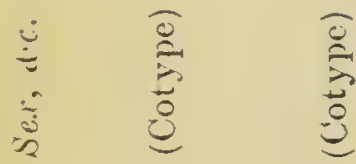

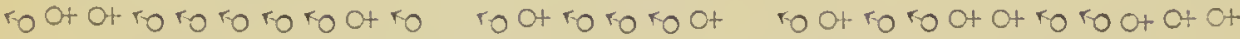

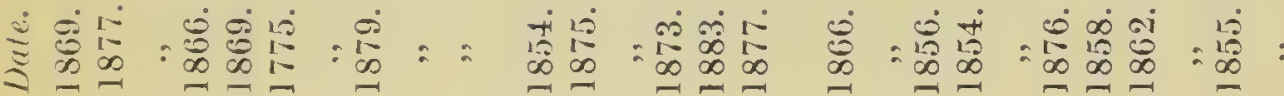

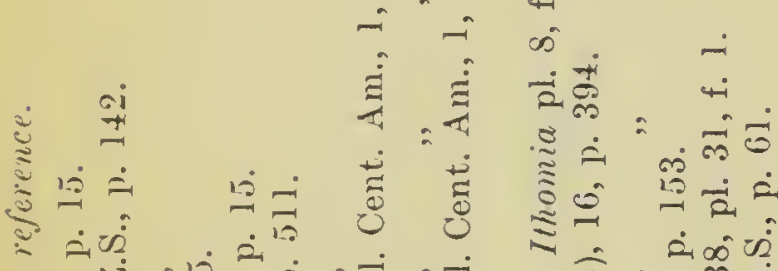

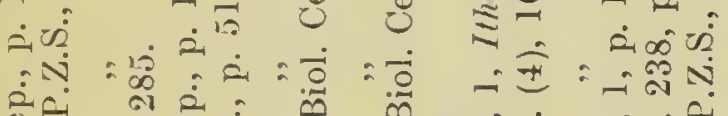

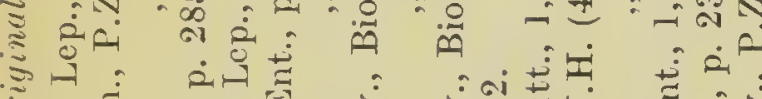

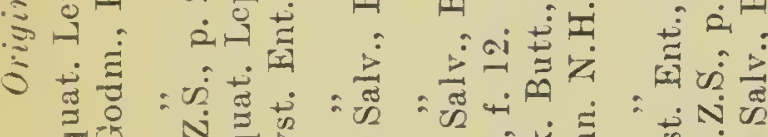

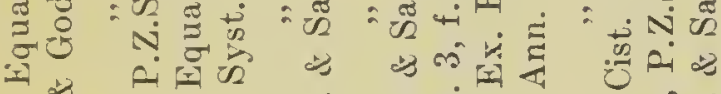

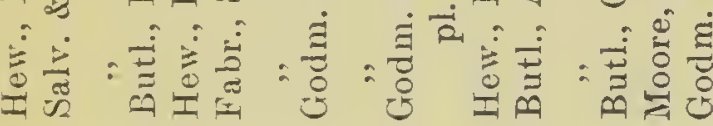

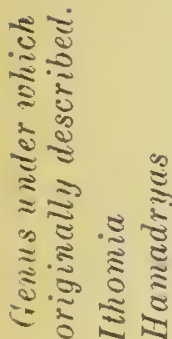

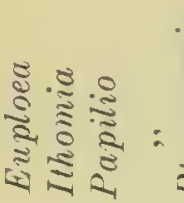

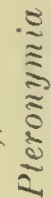

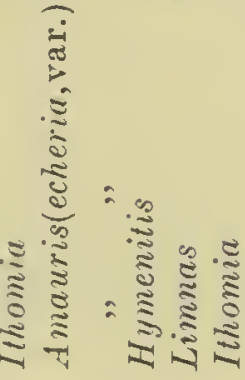

में

तीं +ं की

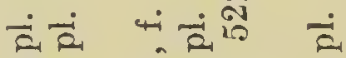
.

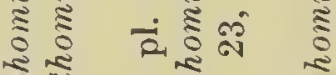
震 i⿱⺈⿵⺆⿻二丨冂口,

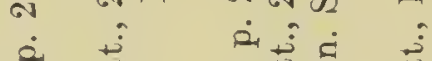

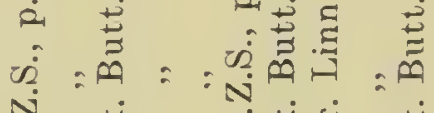

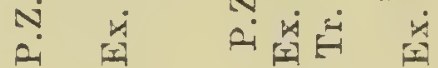
产:

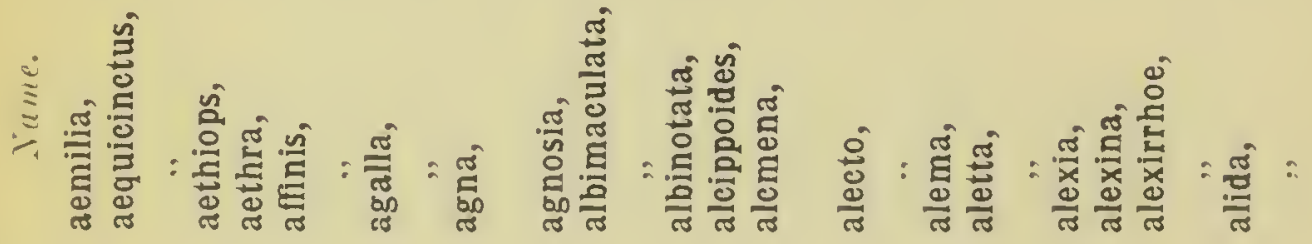
2

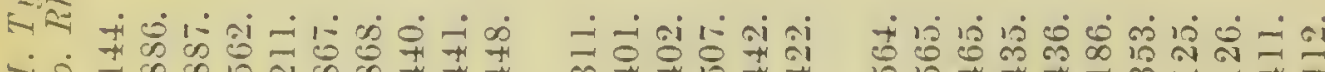

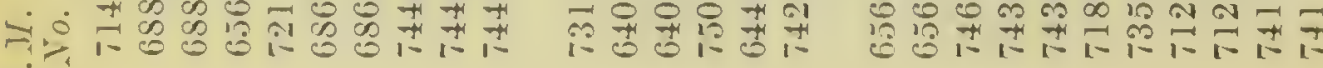
बi 


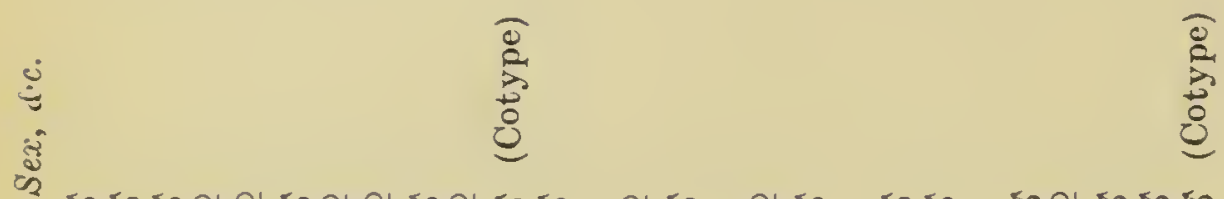

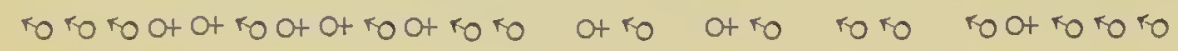

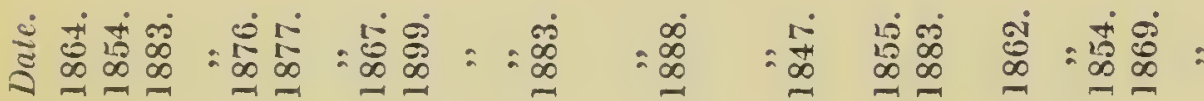

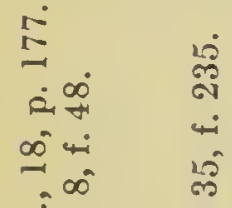

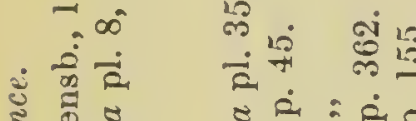

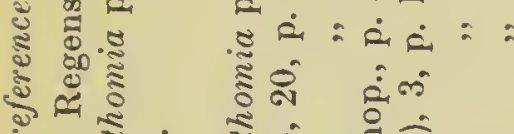

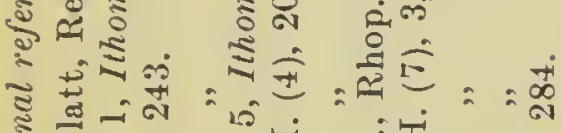

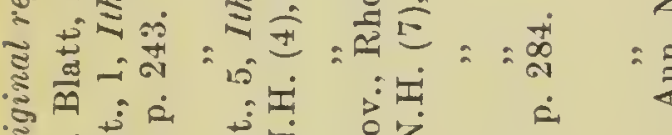

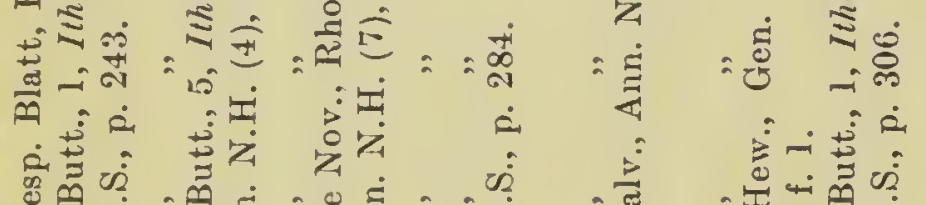

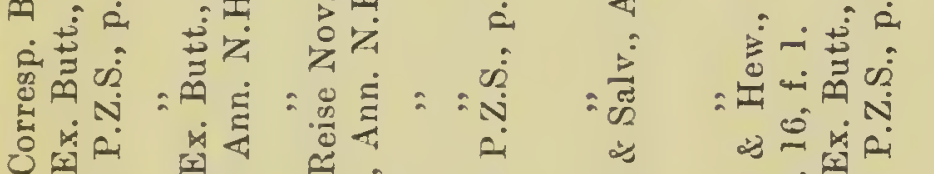

$$
\text { จ }
$$

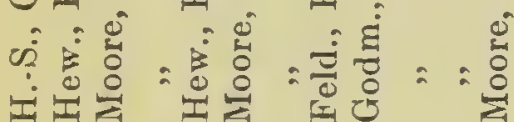

๘

$\approx \stackrel{0}{\circ}$

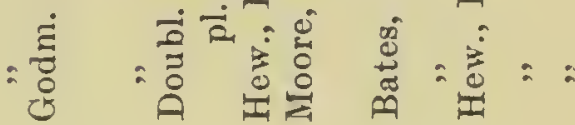

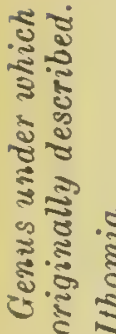

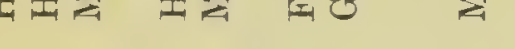

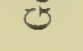

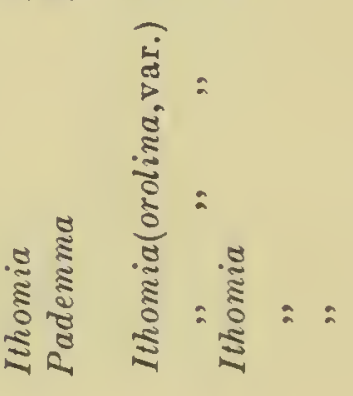

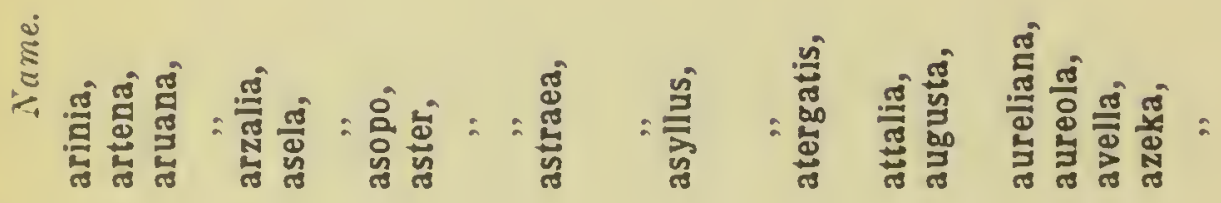

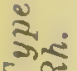

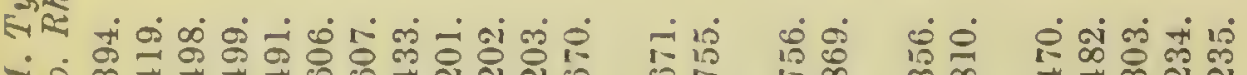

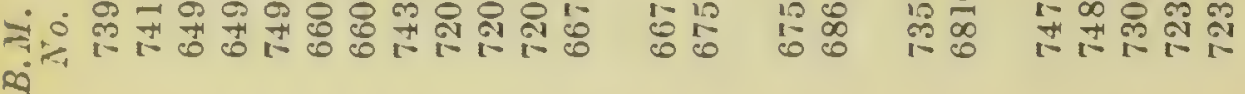




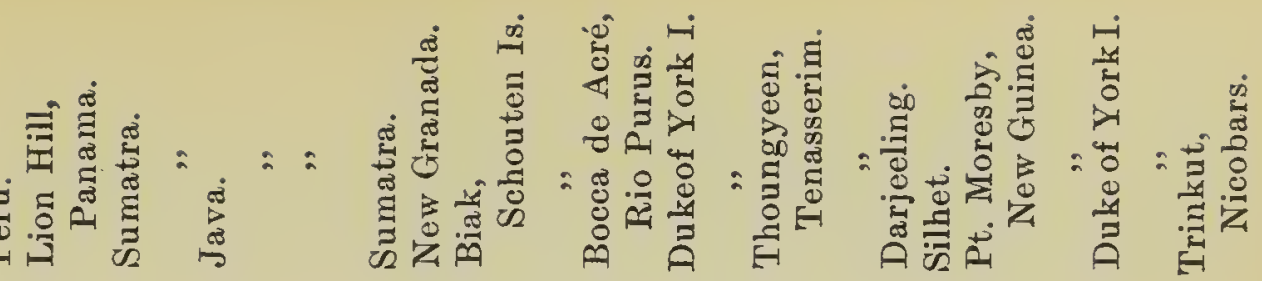

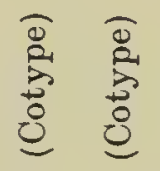

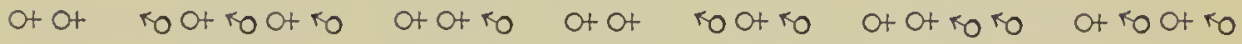

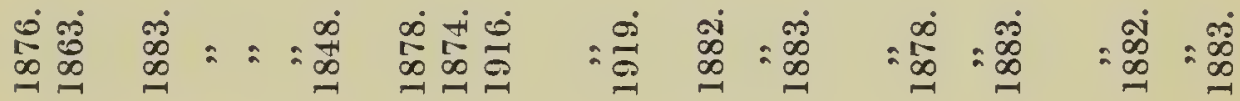

๓

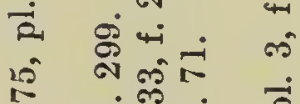

-

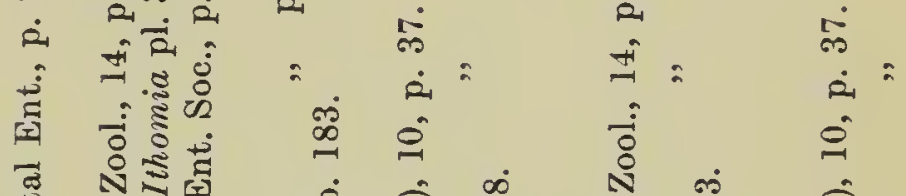

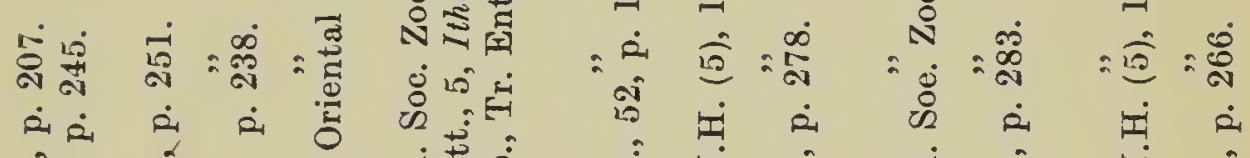

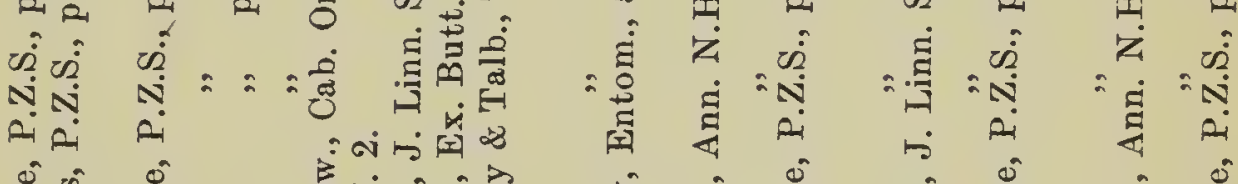

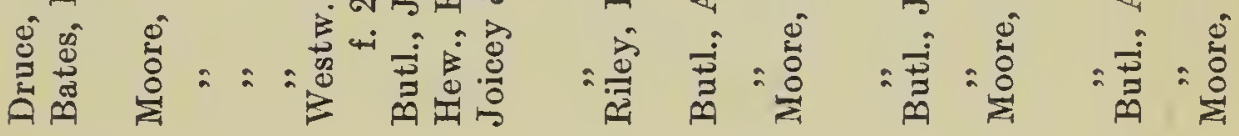

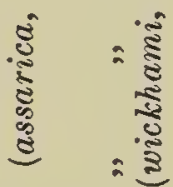

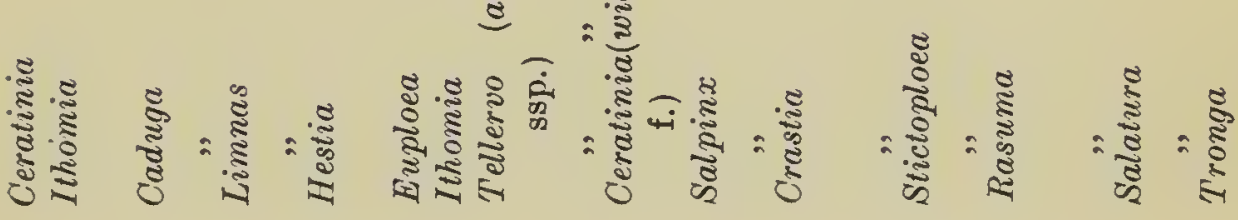

突望

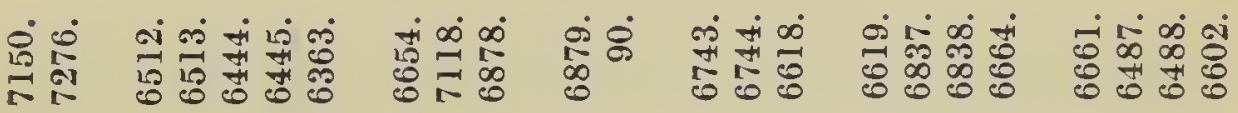


at ro of ro ot ot otro rootrootro ro ot of ro ot ro

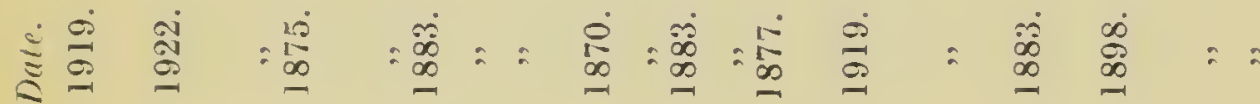

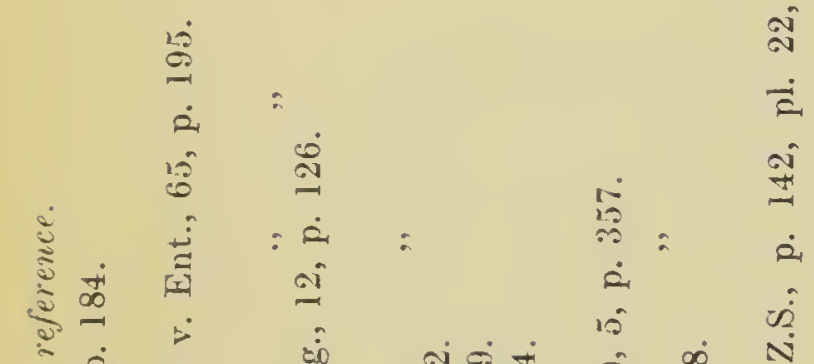

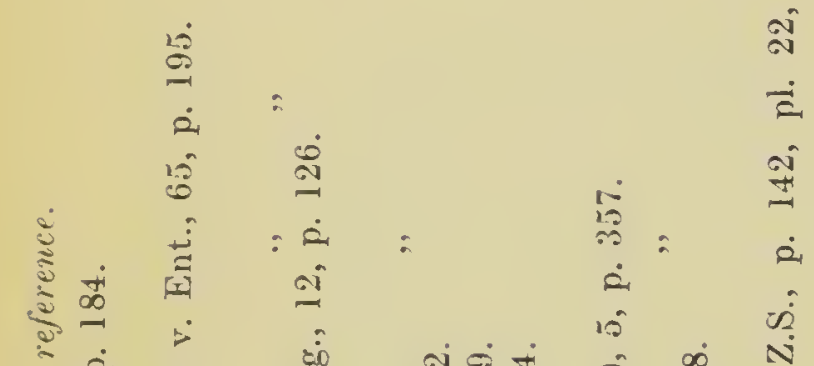

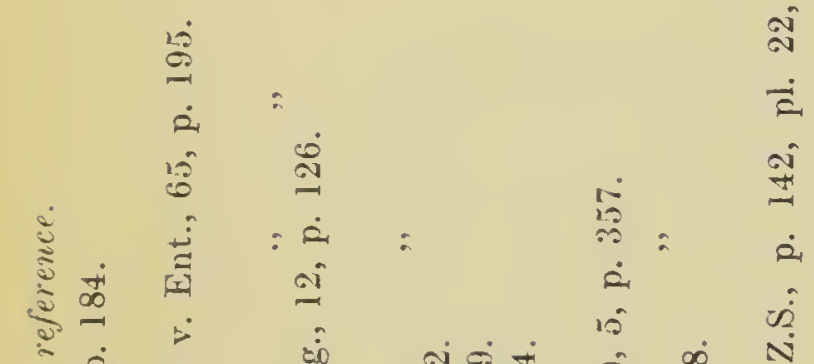

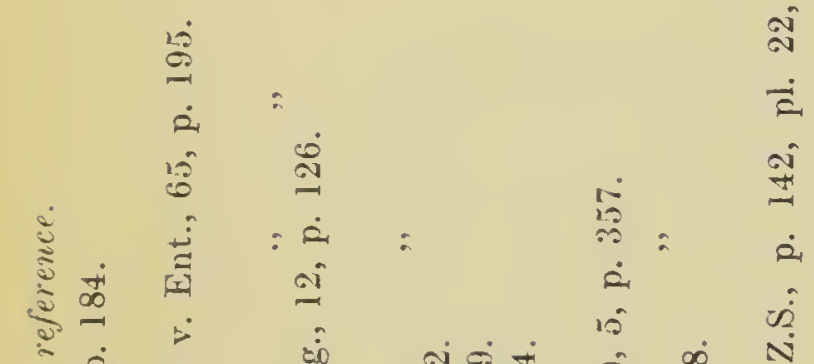

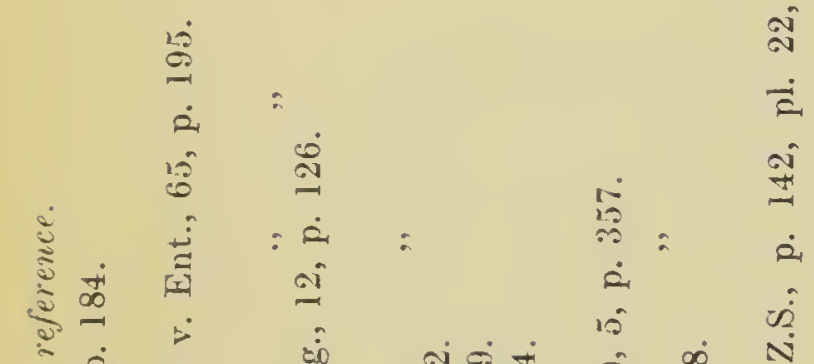

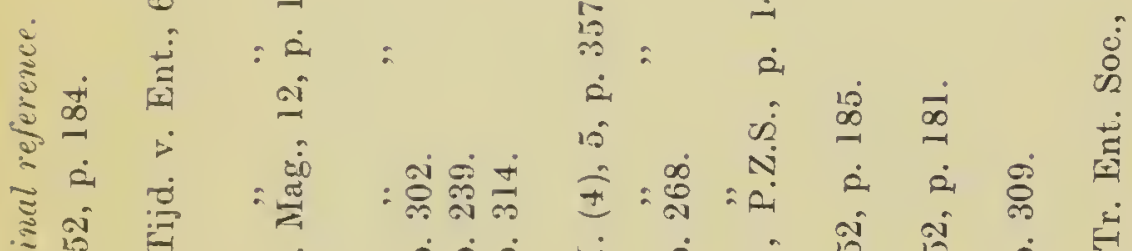

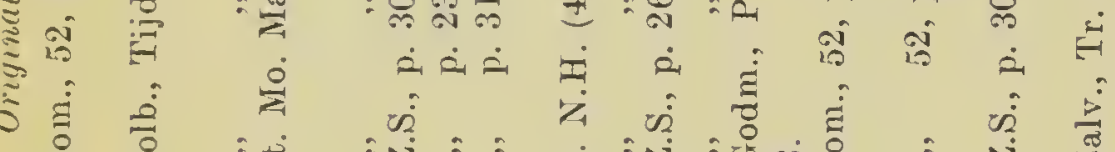

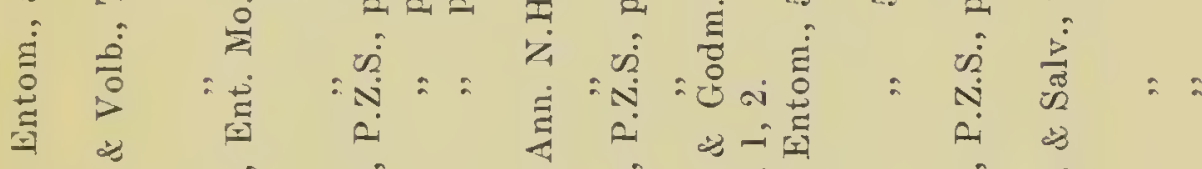

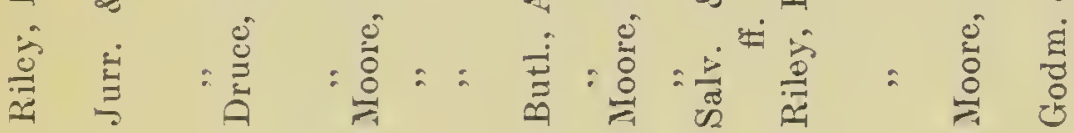
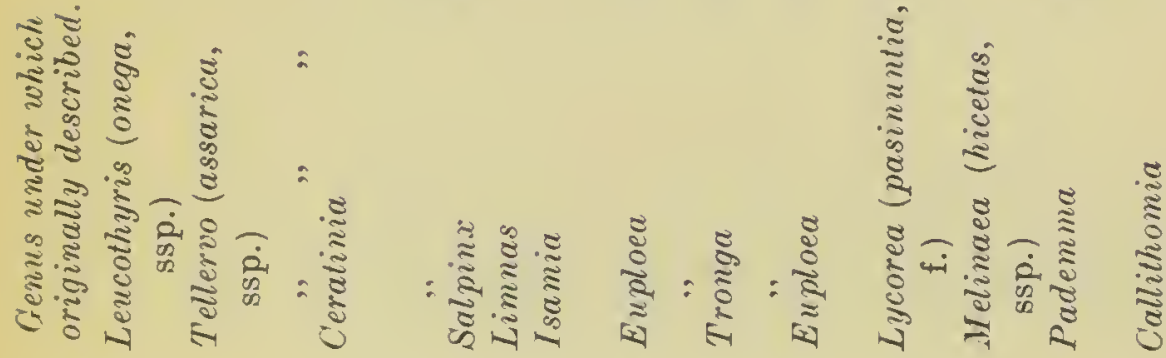

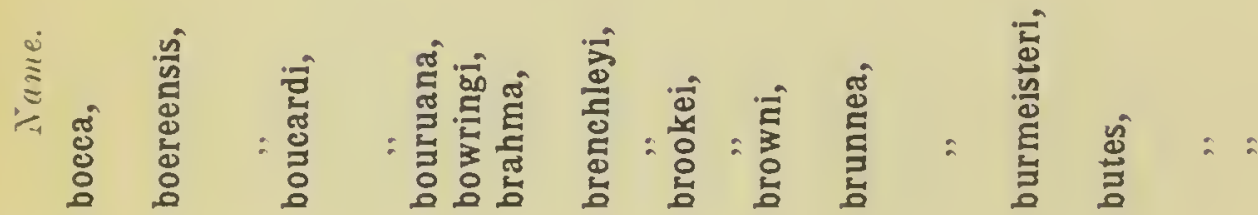

$\stackrel{2}{\sharp}$

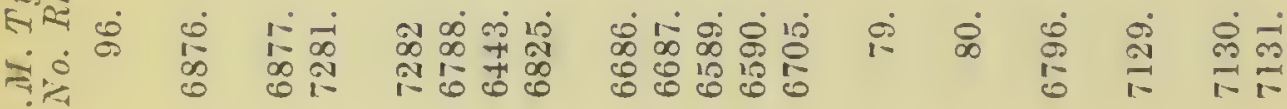
RQ 
商

ot rootro roro rororo ototototro

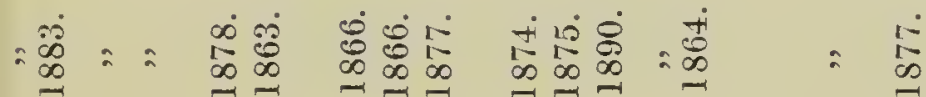

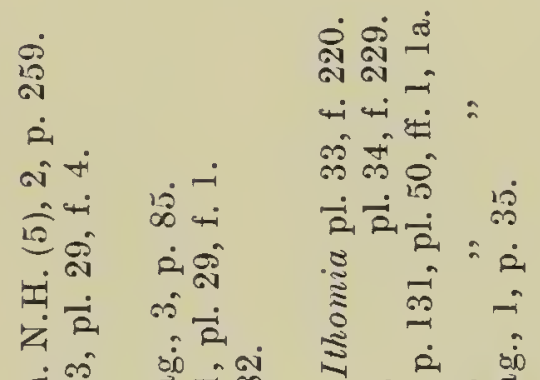

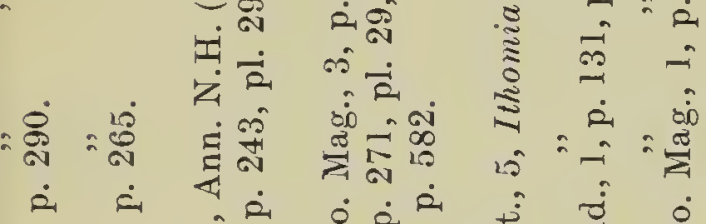

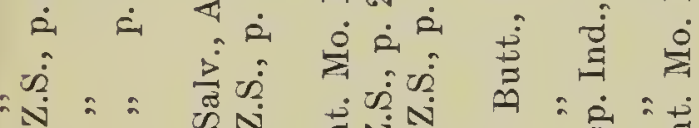

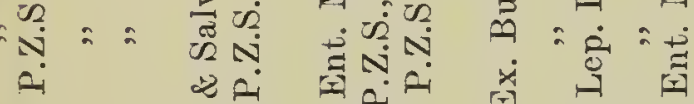

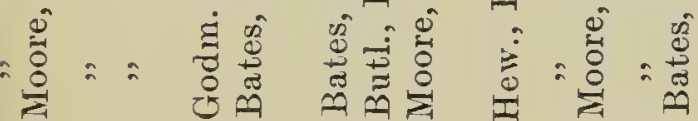

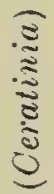

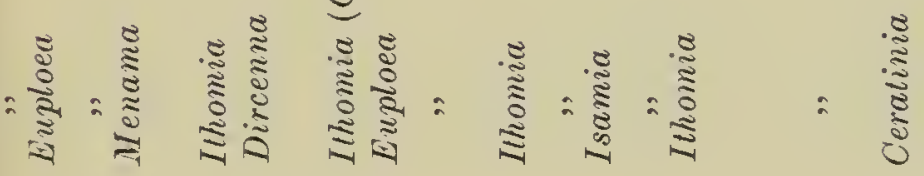

密

.

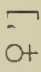

ro ro ro ot ko

$\therefore \quad \begin{aligned} & 10 \\ & 1\end{aligned}$

$1 \quad$ ผั

मा मा

की की

2. त्र

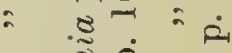

苛

$\dot{2}$

迥

की $=\hat{\text { से }}$

芒芒芒

我主

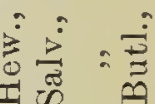

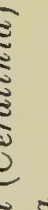

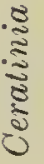

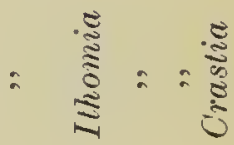

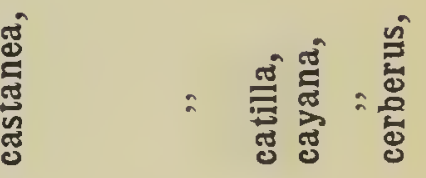

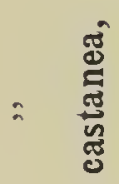

จุ

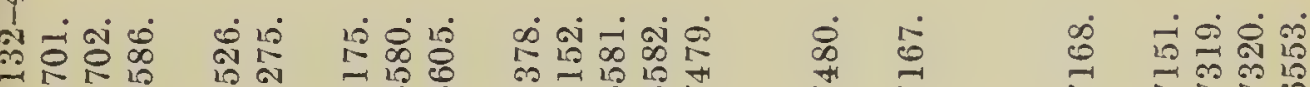

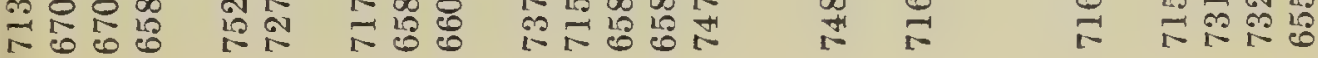




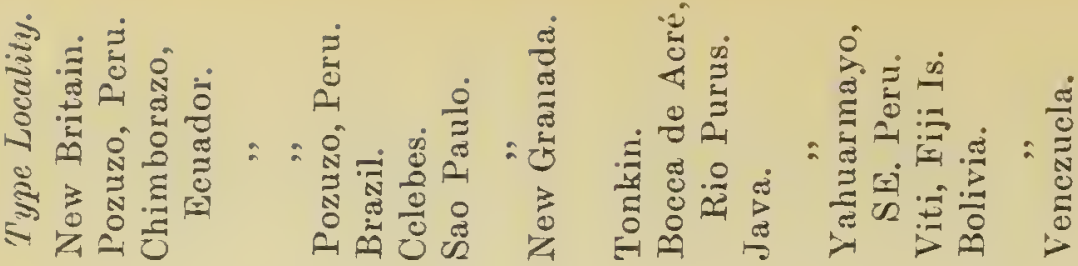

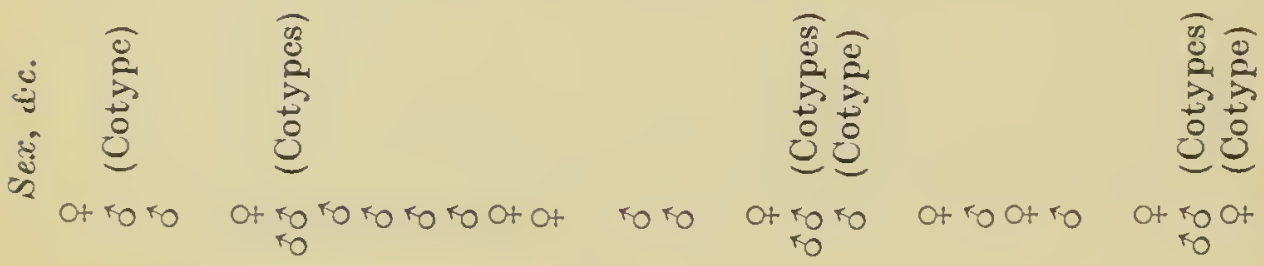

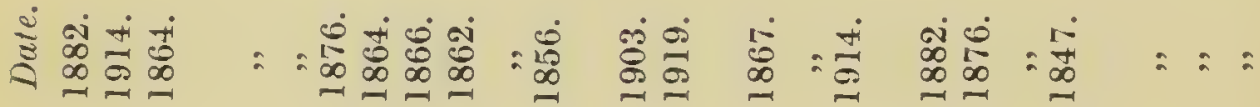

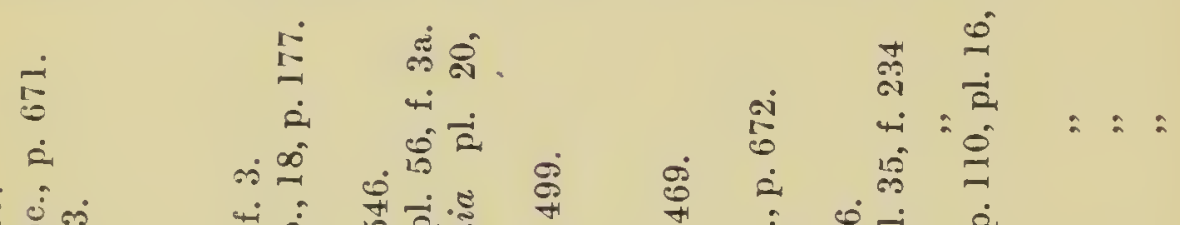

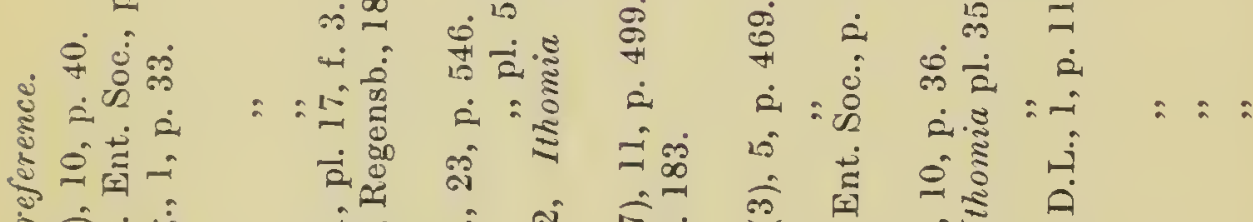

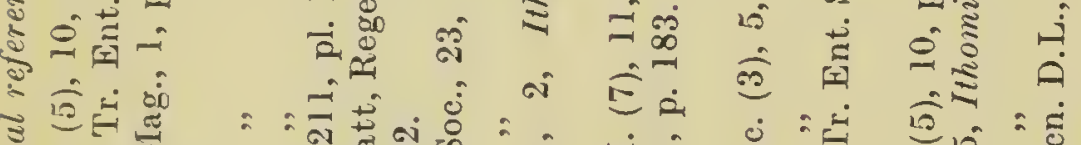

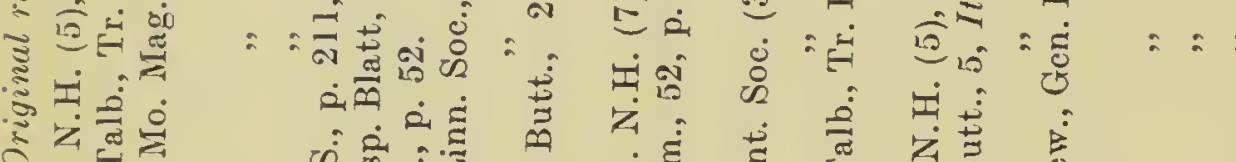

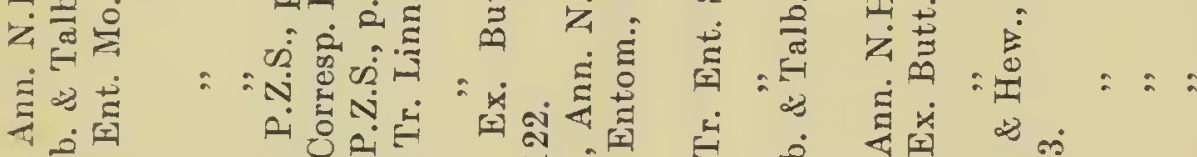

$\therefore$ एँ

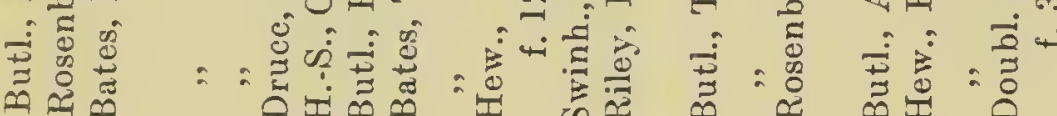

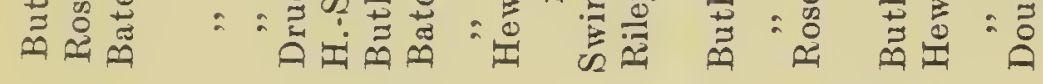
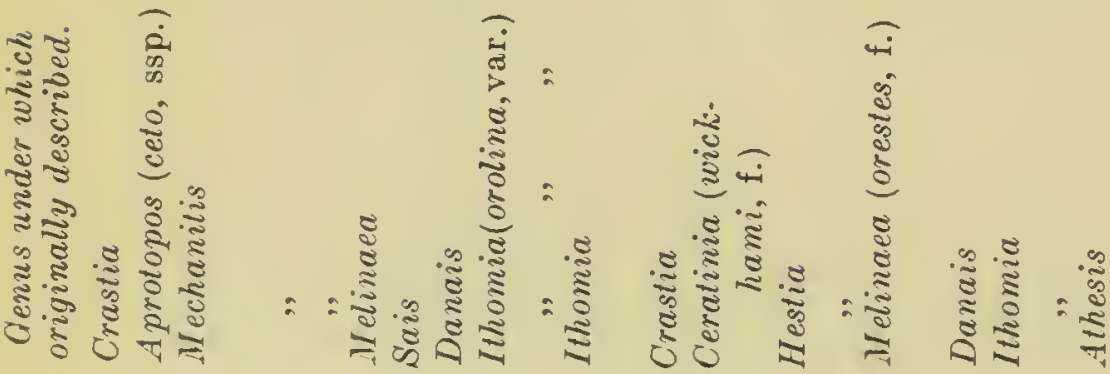

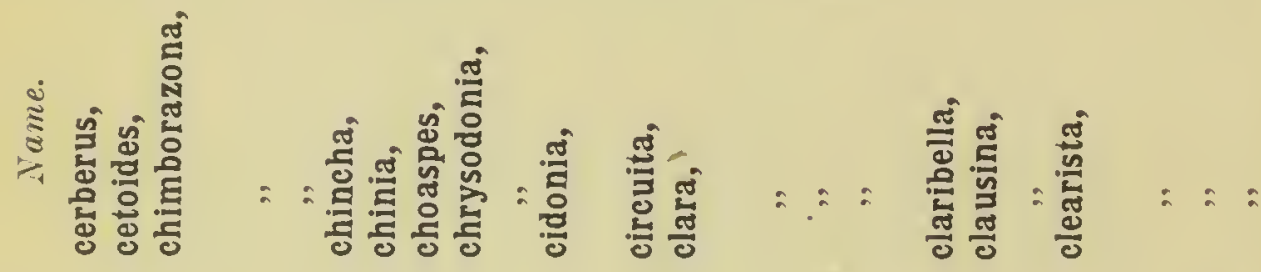

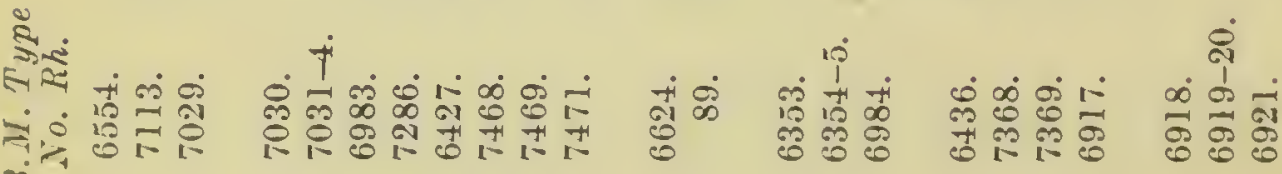
$\infty$ 
$=$ ह

I

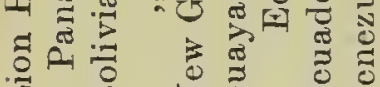

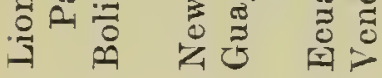

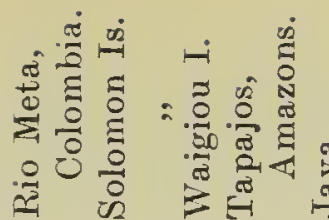

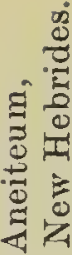

ot

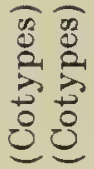

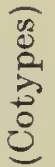

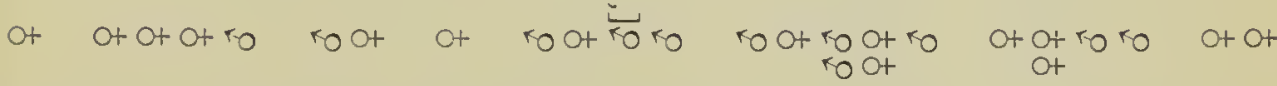

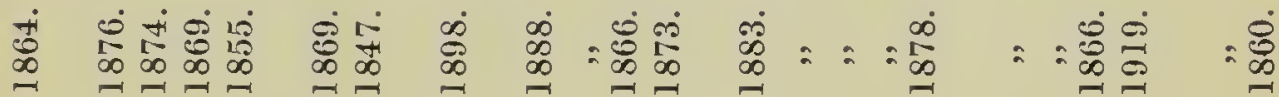

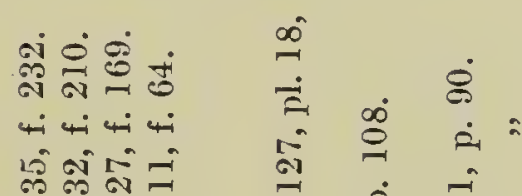

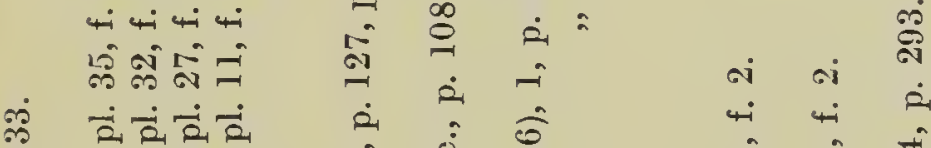

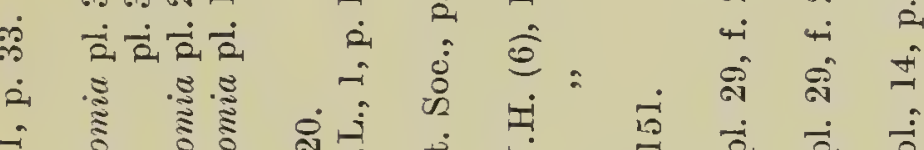

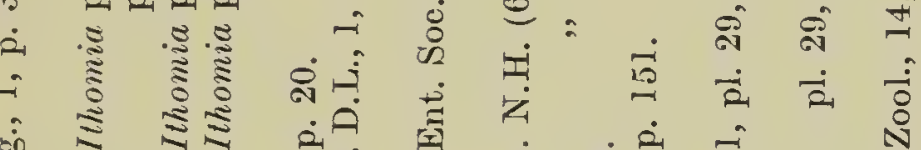

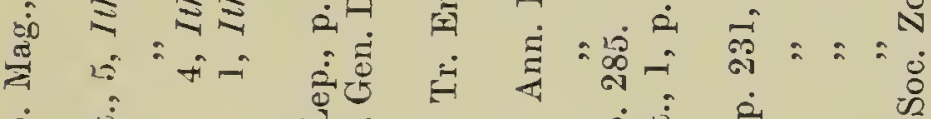

总

荥

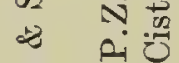

म.

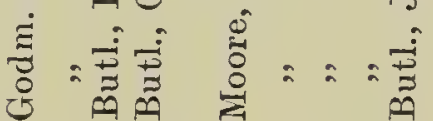

$\frac{\sqrt[3]{\frac{3}{3}}}{\sqrt[3]{3}}$

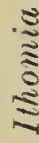

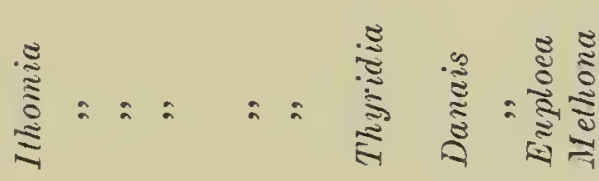

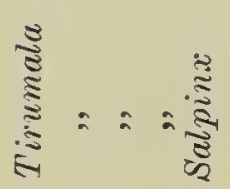

: 


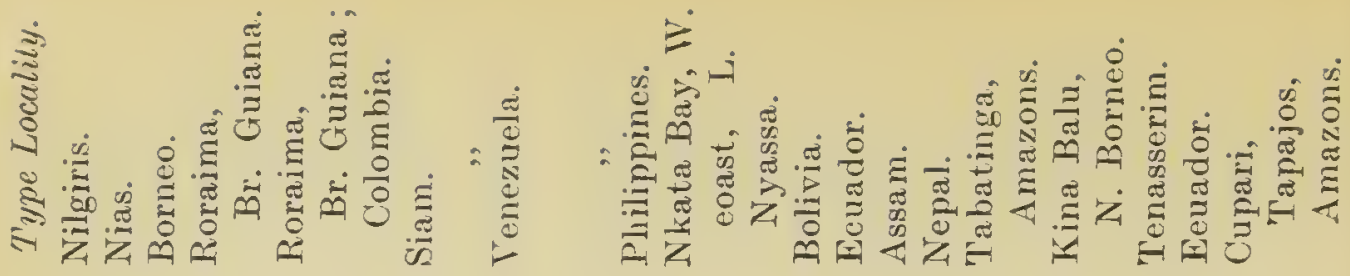

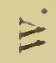

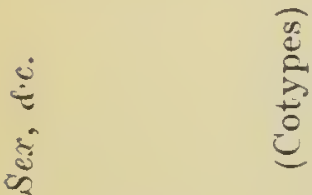

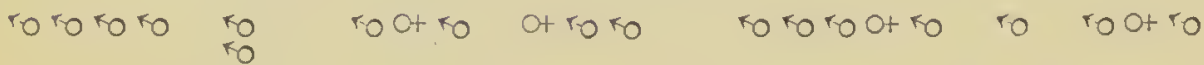

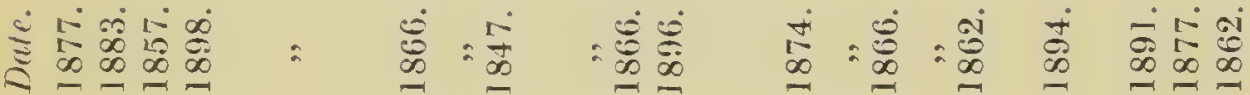

$\div \quad \stackrel{10}{\circ}$

$\stackrel{2}{\stackrel{2}{*} \dot{0}}$

(3)

¿

$\approx$

z $2: 0$

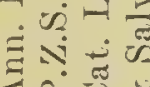

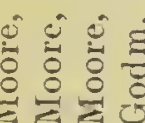

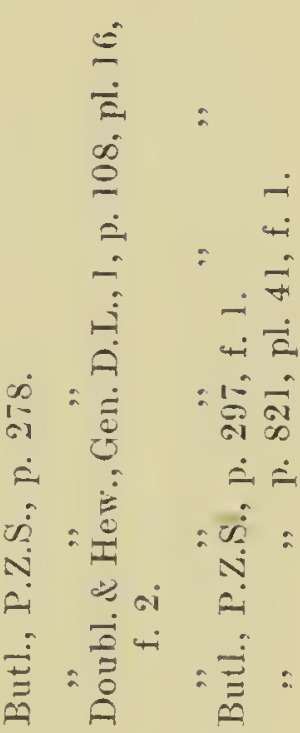

$\frac{2 \pi}{20}$

-

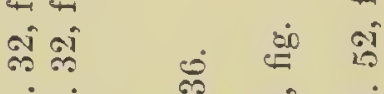

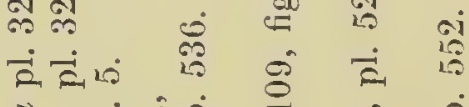

๘

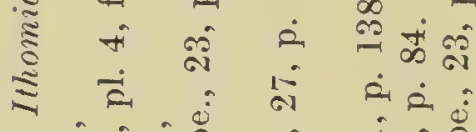

$10^{\circ}$ " $15=0 \begin{aligned} & 0 \\ & 20 \text { ह }\end{aligned}$

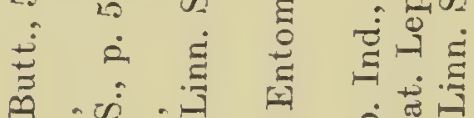

$\because$ ज

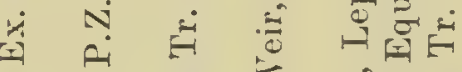

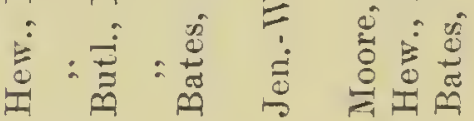

บั)

离

원

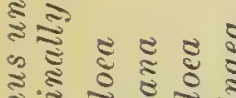

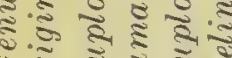

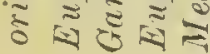

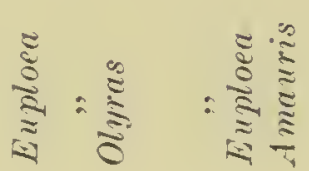

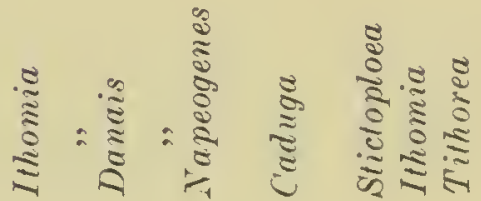

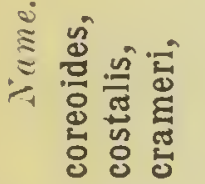

岁:

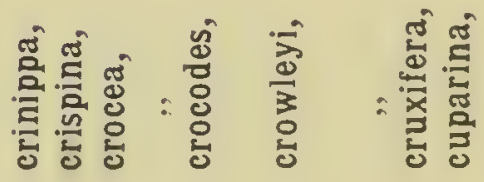

$\stackrel{2}{2}$

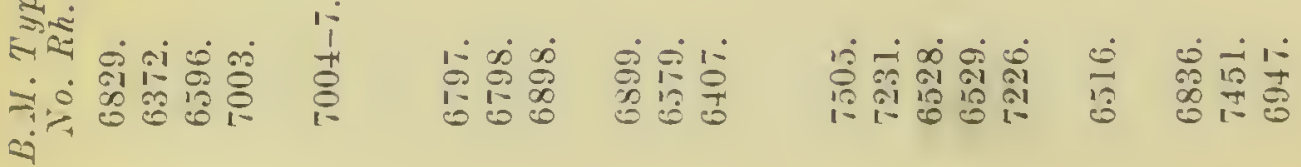




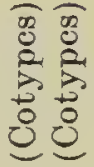

$0+50$

root ro ro

$\mathrm{roO} O \mathrm{OH}$
$\mathrm{roO}$

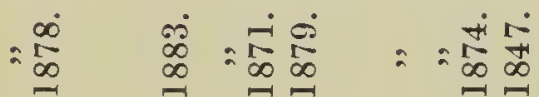

$\stackrel{\infty}{\stackrel{\infty}{\circ}} \stackrel{\infty}{2}$

$+i$

กิ

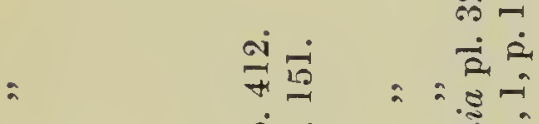

$\dot{\sim} \dot{2}$

.8

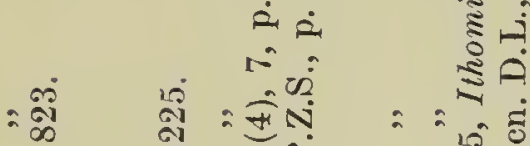

$\therefore$ व $\dot{x}^{2}$

$=\frac{\infty}{\infty} \quad \therefore=2 \frac{1}{2}$

\&

0
0
0
0
0 छ

$\therefore=\frac{2}{30}$

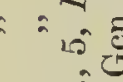

要

คิ 我出证

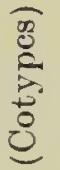

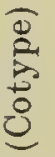

ro rorootromot roro atroto

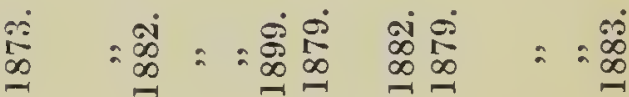

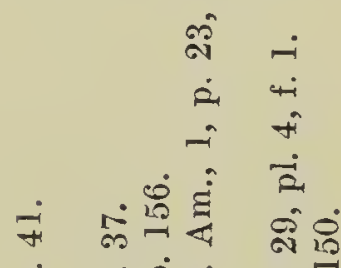

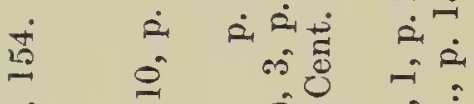

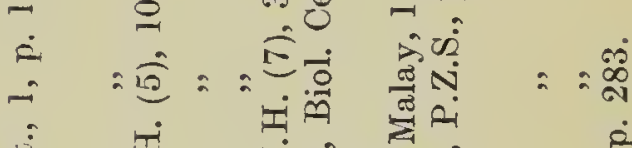

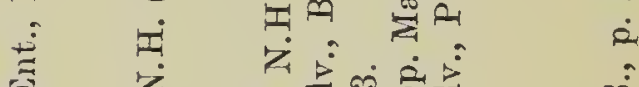

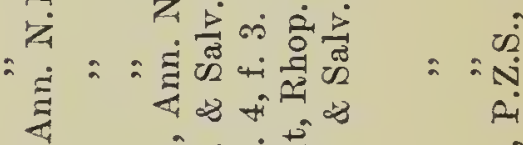

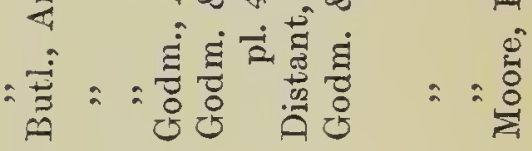

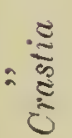<smiles></smiles>

$\stackrel{2}{\bar{*}}$

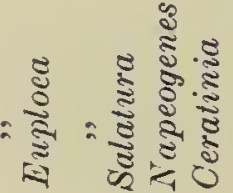

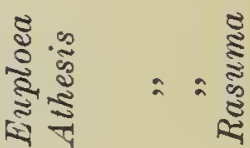

:

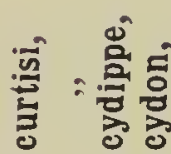

$=2$ 莺莺

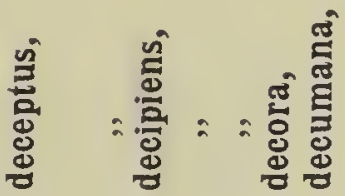

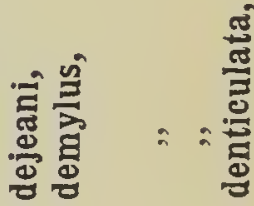

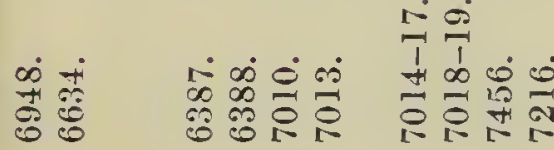

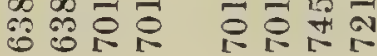

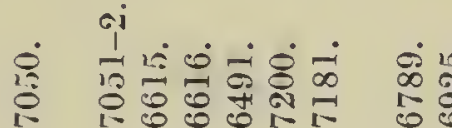

$\infty$ N $\infty$ i 
कूष

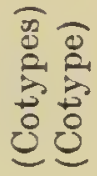

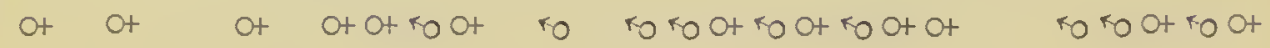

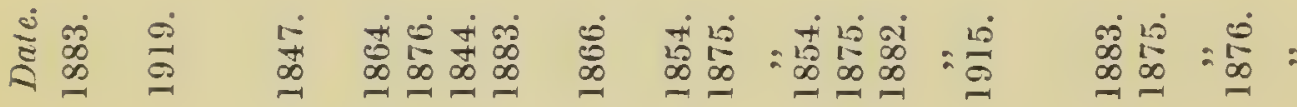

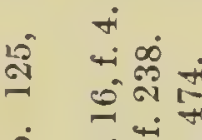

¿ँّ

के क्ष

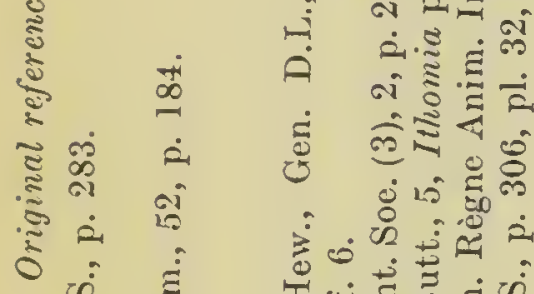

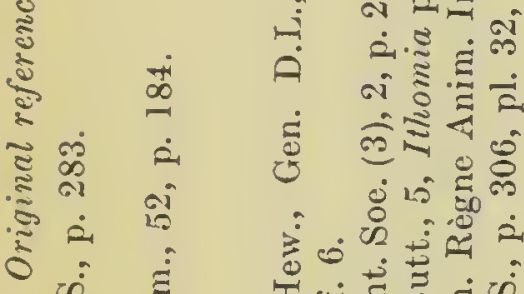

ìं

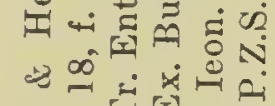

$\stackrel{0}{0} \stackrel{0}{0}$

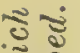

:

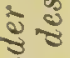

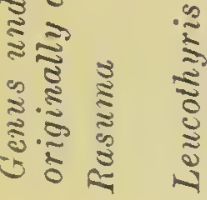

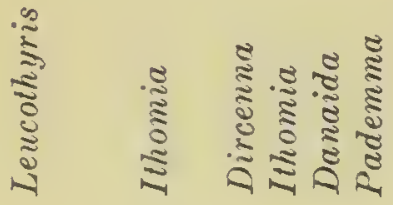

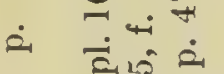

$=\quad \infty 150$ -

$\dot{0}$

๙

में 10 ले

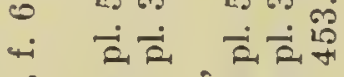

बิ

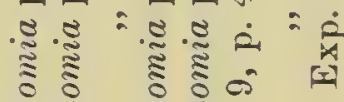

₹

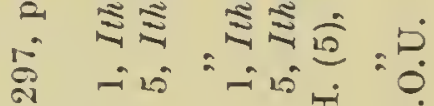

बे

मे

‥

ai

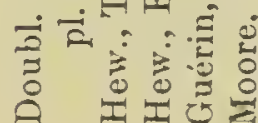

I

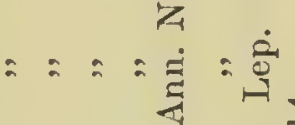

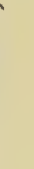

$\infty$
ลิ
ลิ

4 :

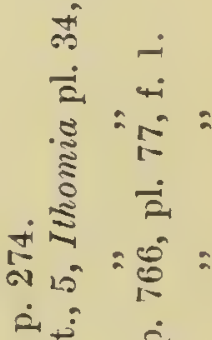

幽

की

计

芜:

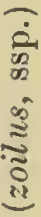

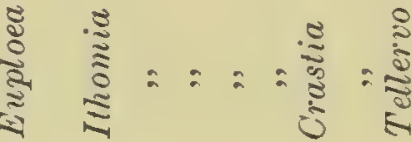

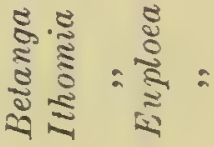

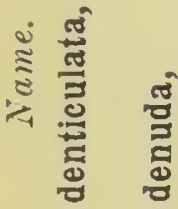

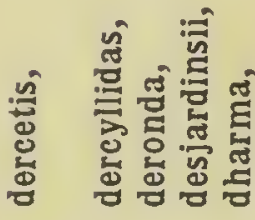

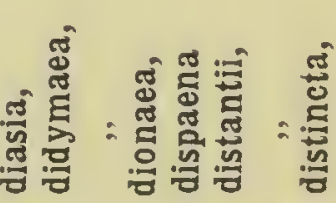

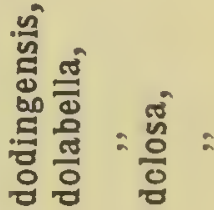

$\stackrel{1}{2}$

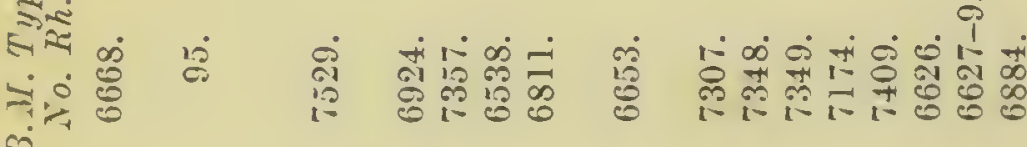




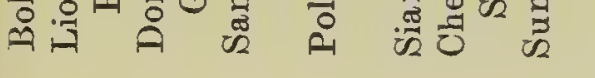

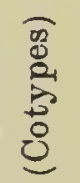

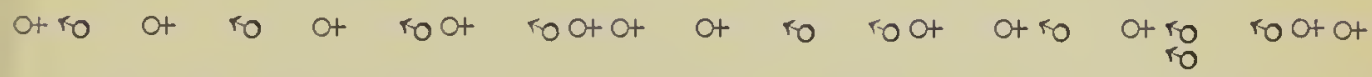

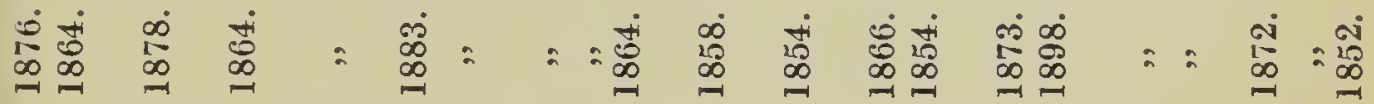

:

in

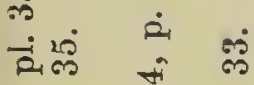

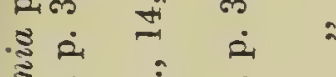

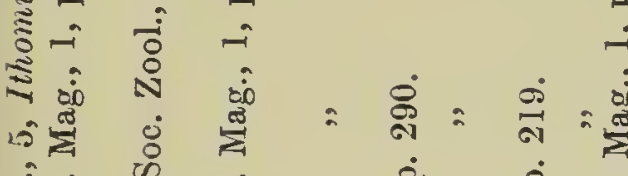

苛官 घ

我若

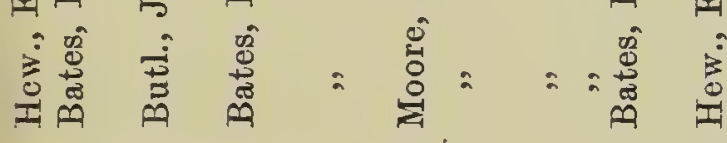

भin

में

งิ तू के

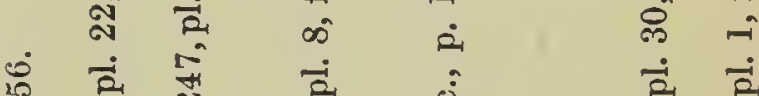

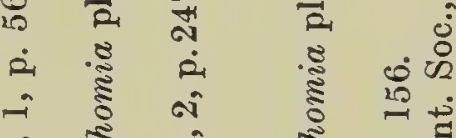

का ले बे

离 मा

ज एं के नि

टें क्षे

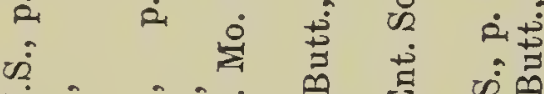

空

ن.

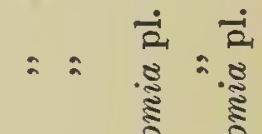

送

ت ค F

再

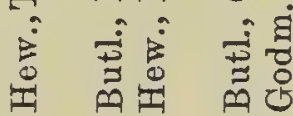

离

蓄

ค

i

$\therefore=\sum_{z=1}^{\infty} a$

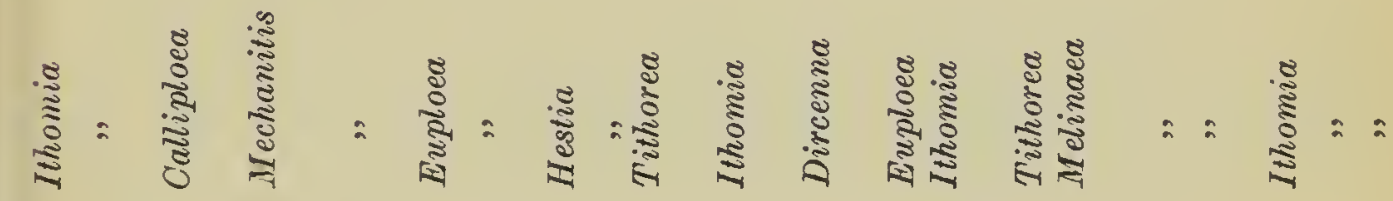

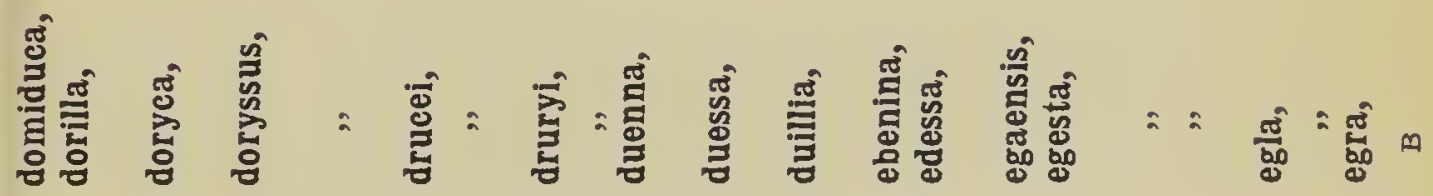

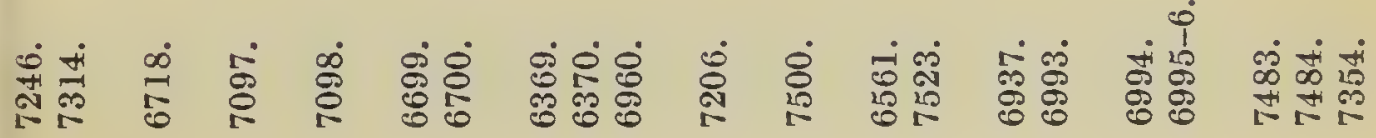




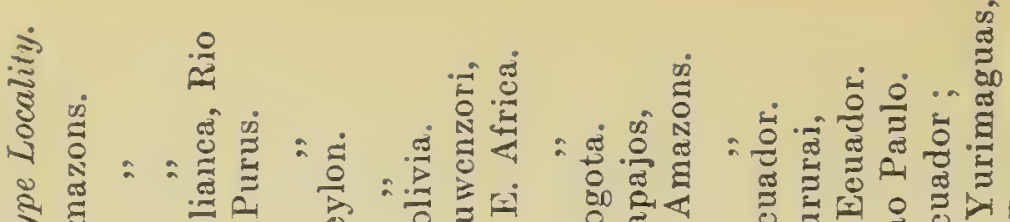

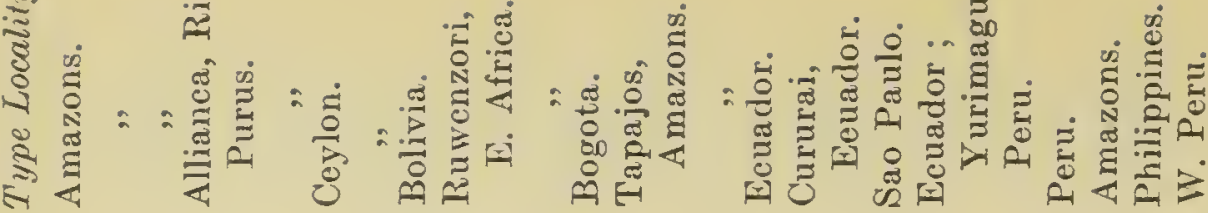

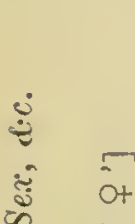

rotoro ro otrootroro
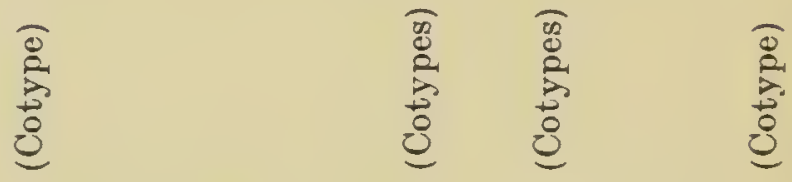

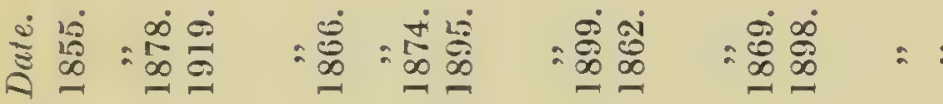

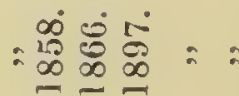

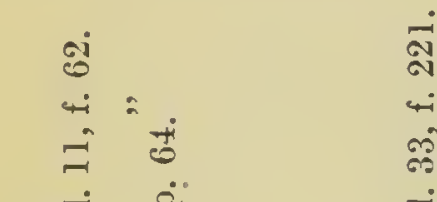

ลั่

4

i $\dot{\overrightarrow{0}} \dot{2}$

ध

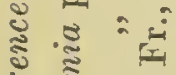

$\therefore \dot{a}: \dot{a}$

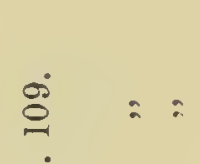

$=\stackrel{\infty}{\text { ज. }}$

तो के

¿

इ

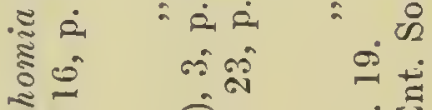

$\sim$ च

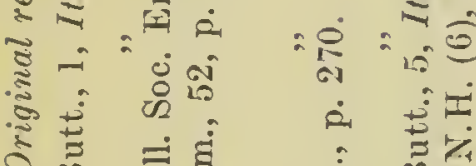

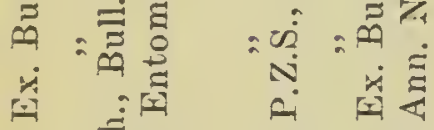

की

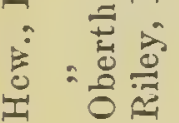

a $\Rightarrow$

E0ं

홀

क

芒

范

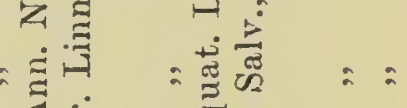

a

से

त्) कि

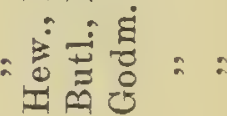

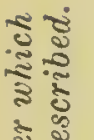

ఫे ญ్

离

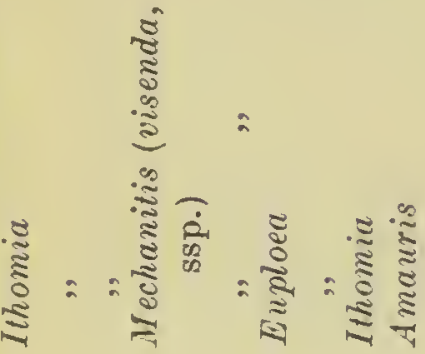

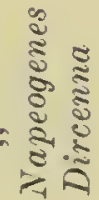

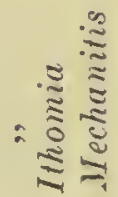

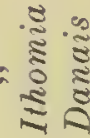




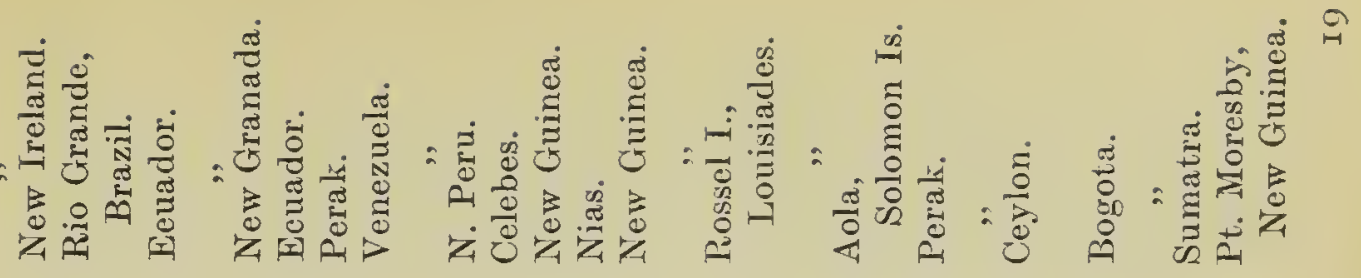

裹

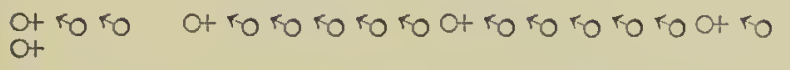

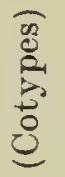

ro ro rootro ot ot of ro

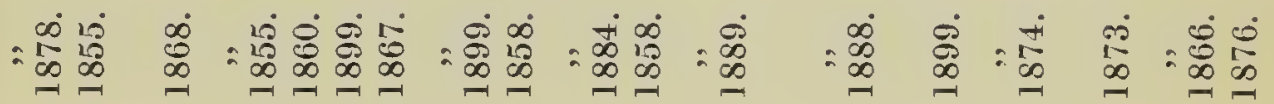

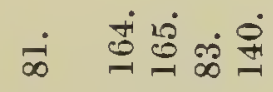

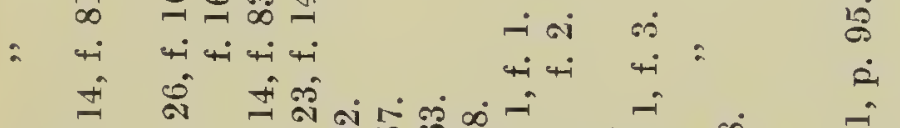

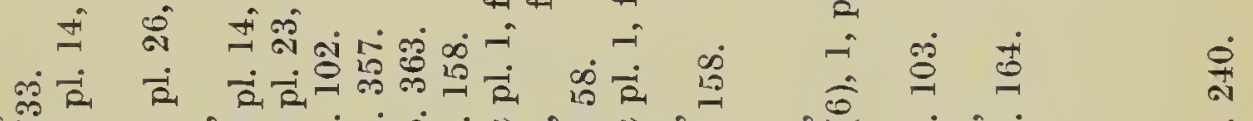

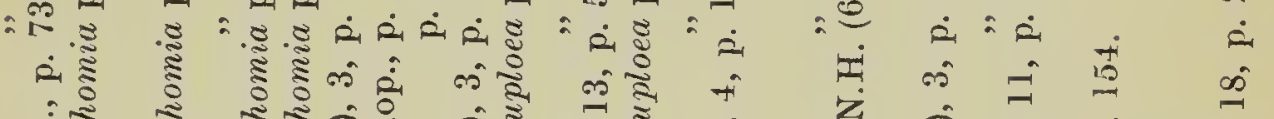

की

话 +

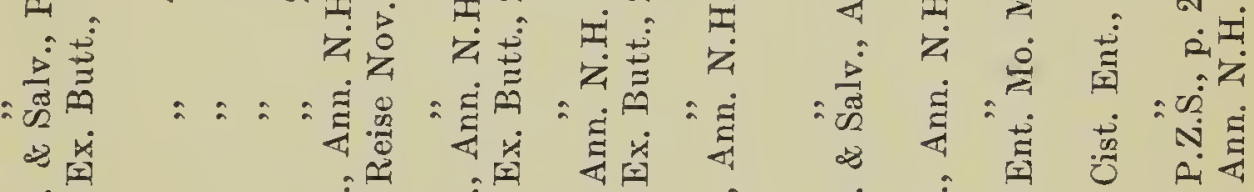

:

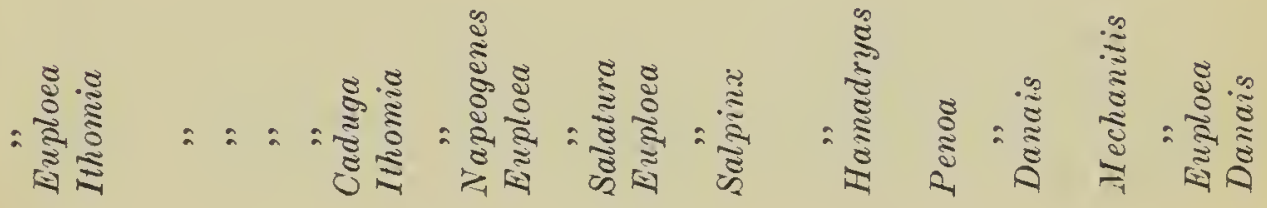

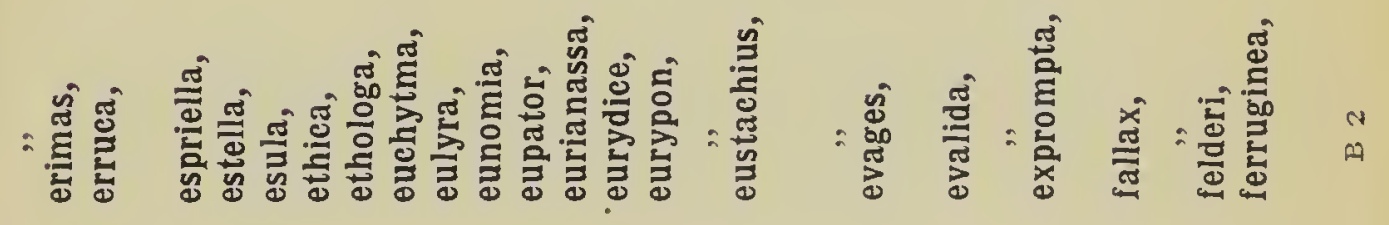

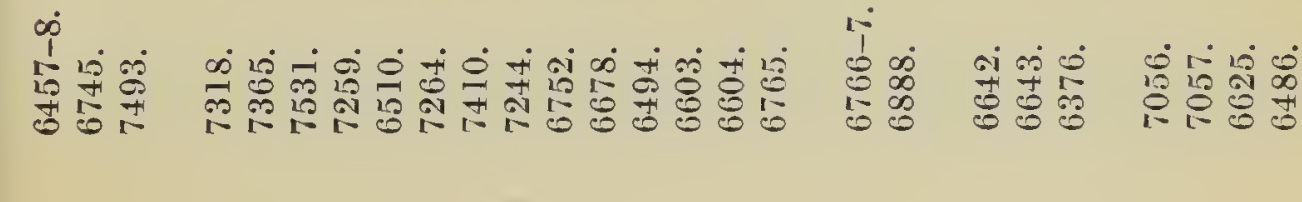

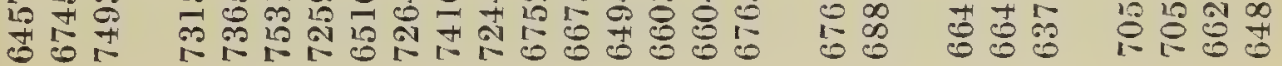




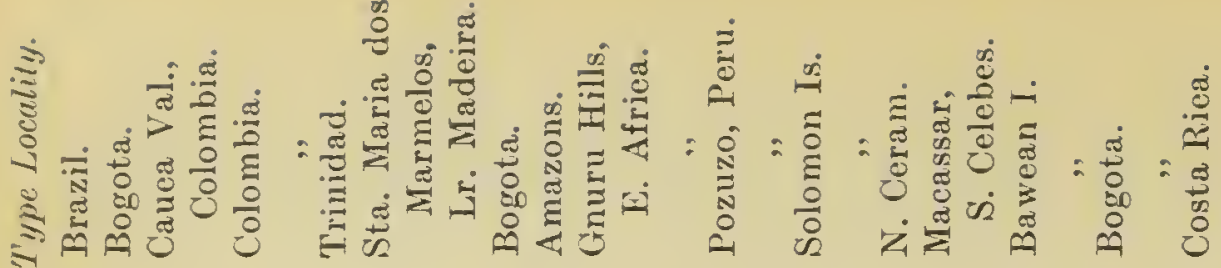

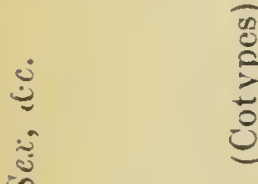

rootro root root

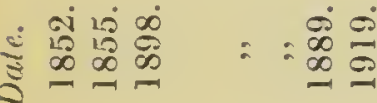

सं 40

คั ติ

료:

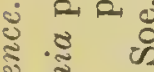

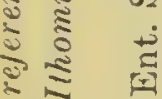

$\vec{s}-\dot{m}$

$\doteq \frac{\vdots}{2}=$

$\Rightarrow$ के

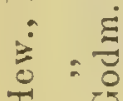

II 5

I क

$\dot{2} \dot{2}$

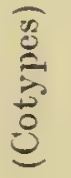

root ro

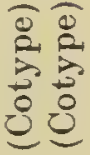

rorootrootroro rootrootro ot
(1.

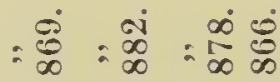

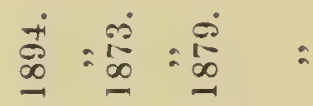

$\stackrel{4}{7}$

के

i.

$\therefore=0$

$\pm \equiv$

$\dot{0} \sim \dot{0}$

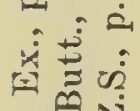

$0_{i}^{\infty}$

$\therefore \dot{\Xi}$

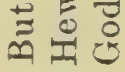

$\dot{2} \dot{2}$

$\therefore \quad-$

岁 घं

ค่

$\dot{a} \hat{0} \quad \dot{0}+\dot{0}$

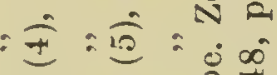

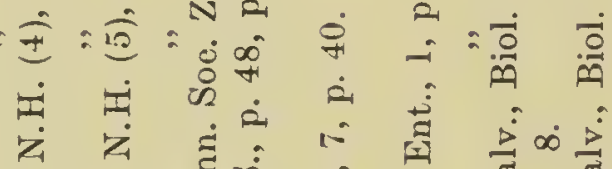

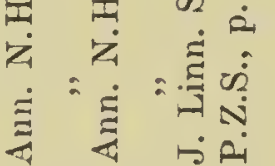

竞:

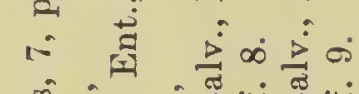

艄:

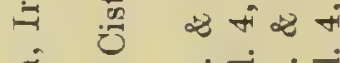

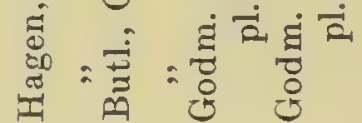

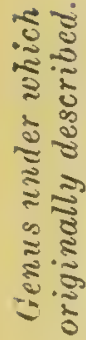
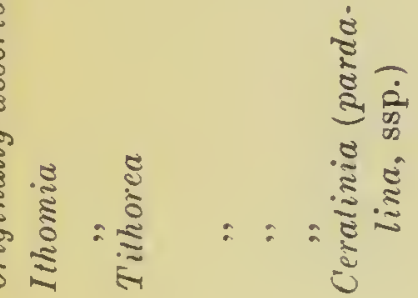

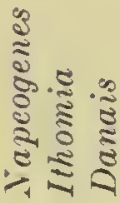

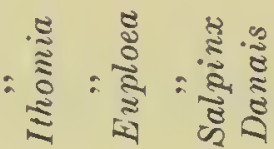

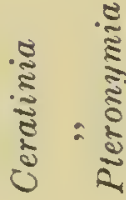

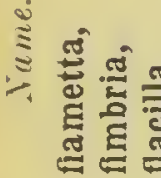

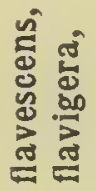

를 용

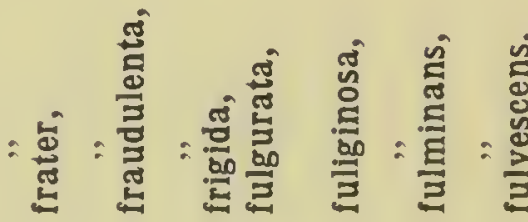

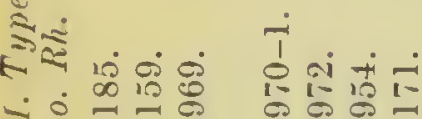

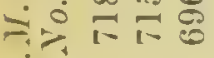

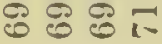

$\infty_{i=1}^{\infty} \dot{0}_{i=1}^{-1}$

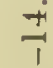

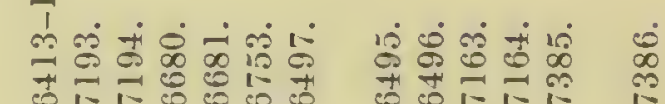




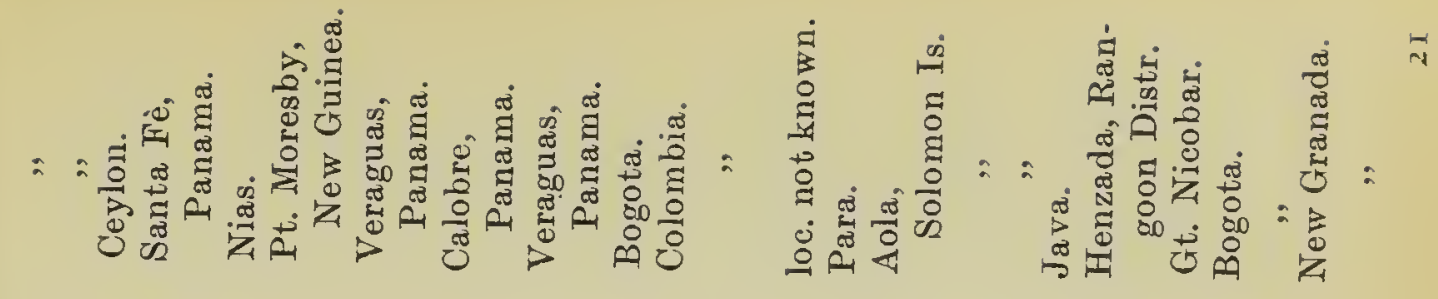

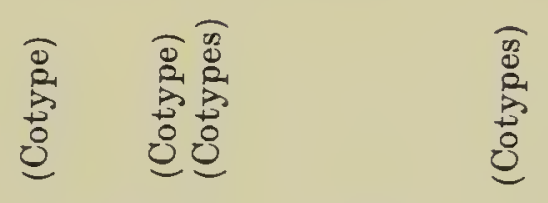

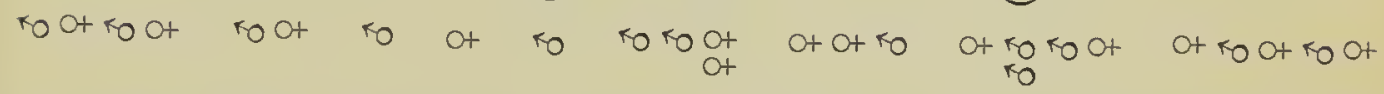

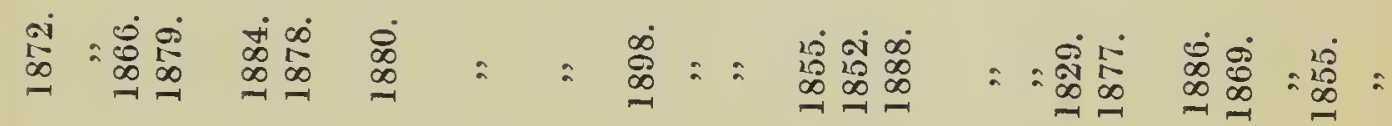

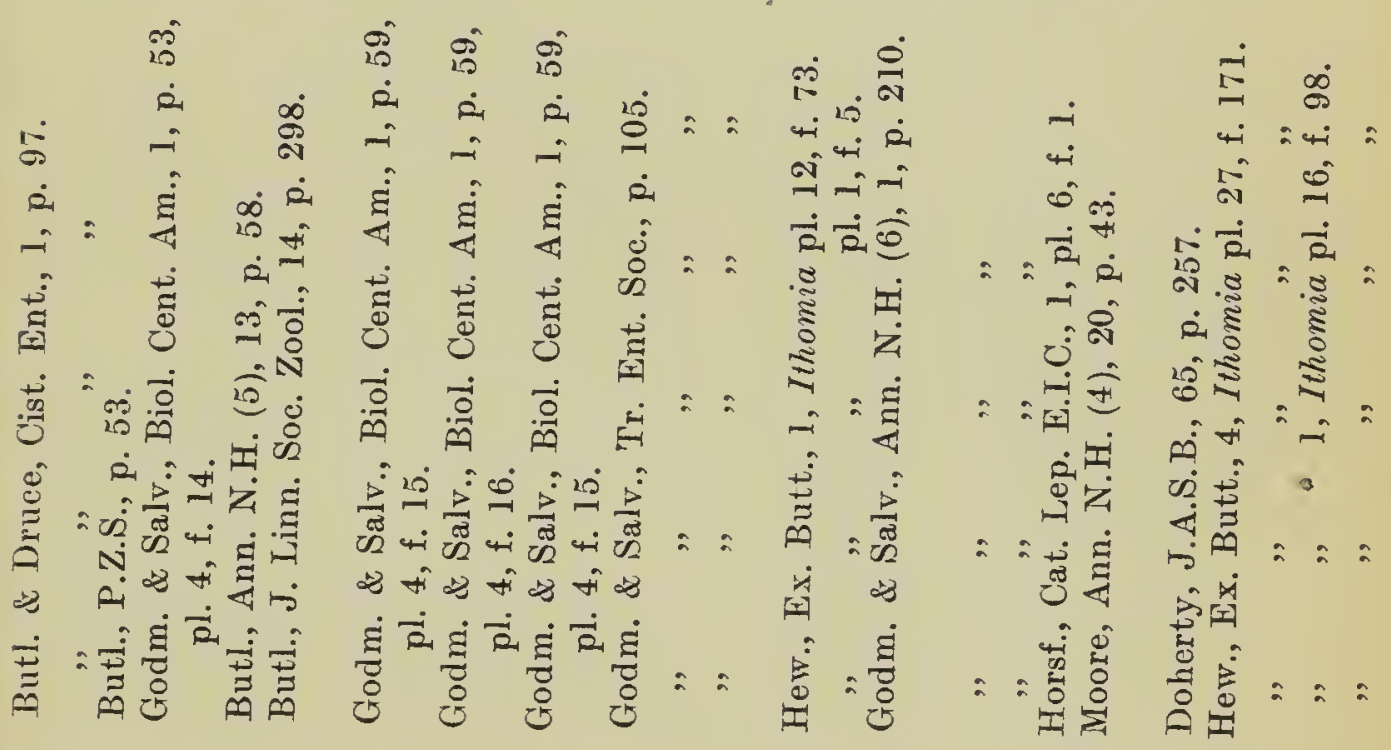

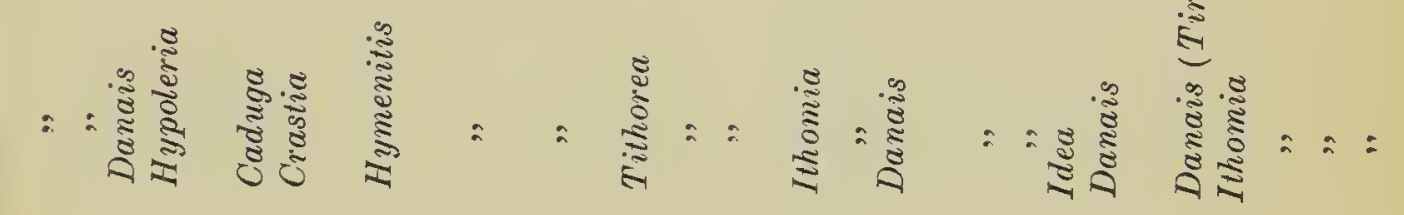

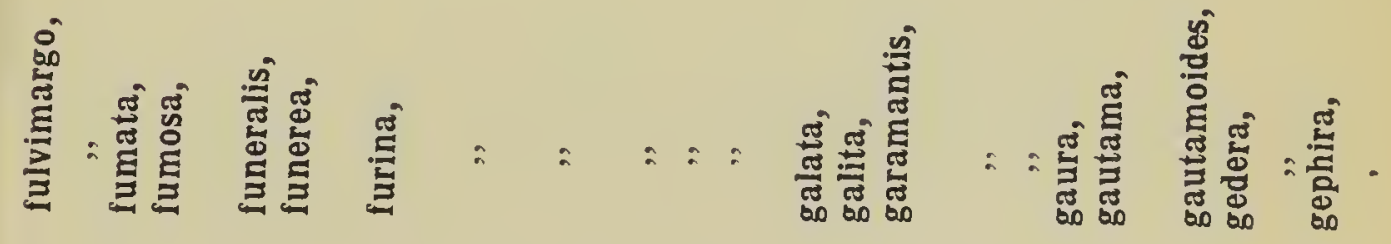

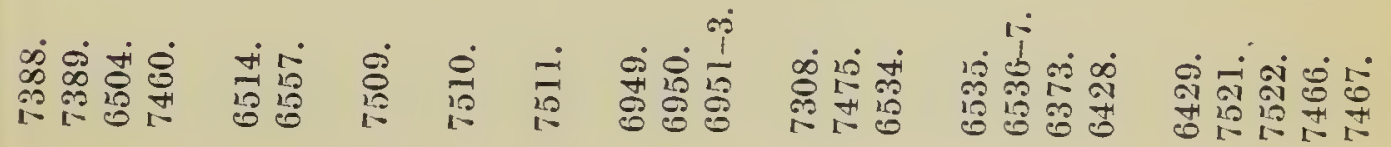




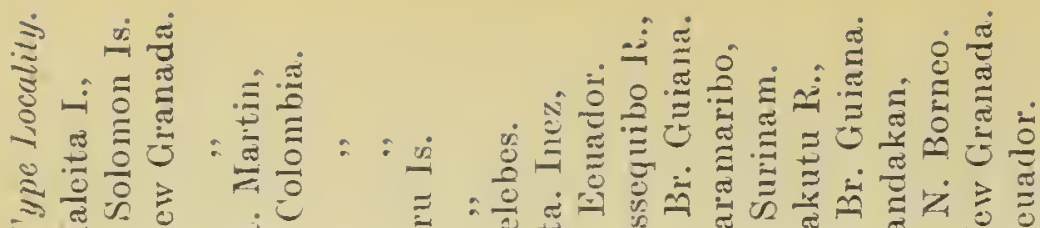

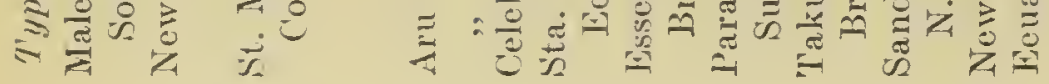
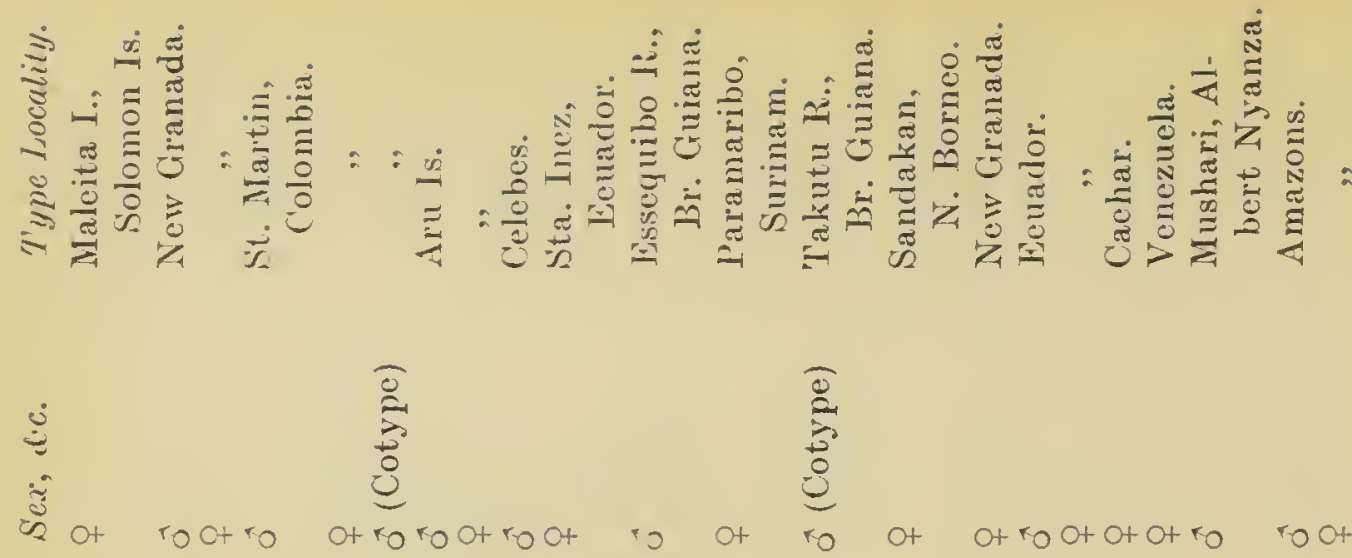

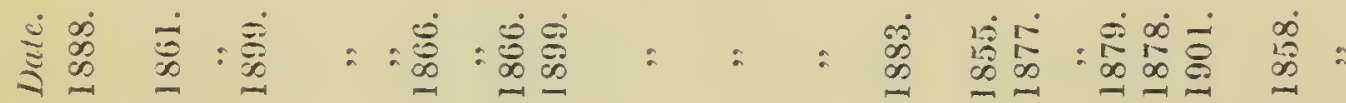

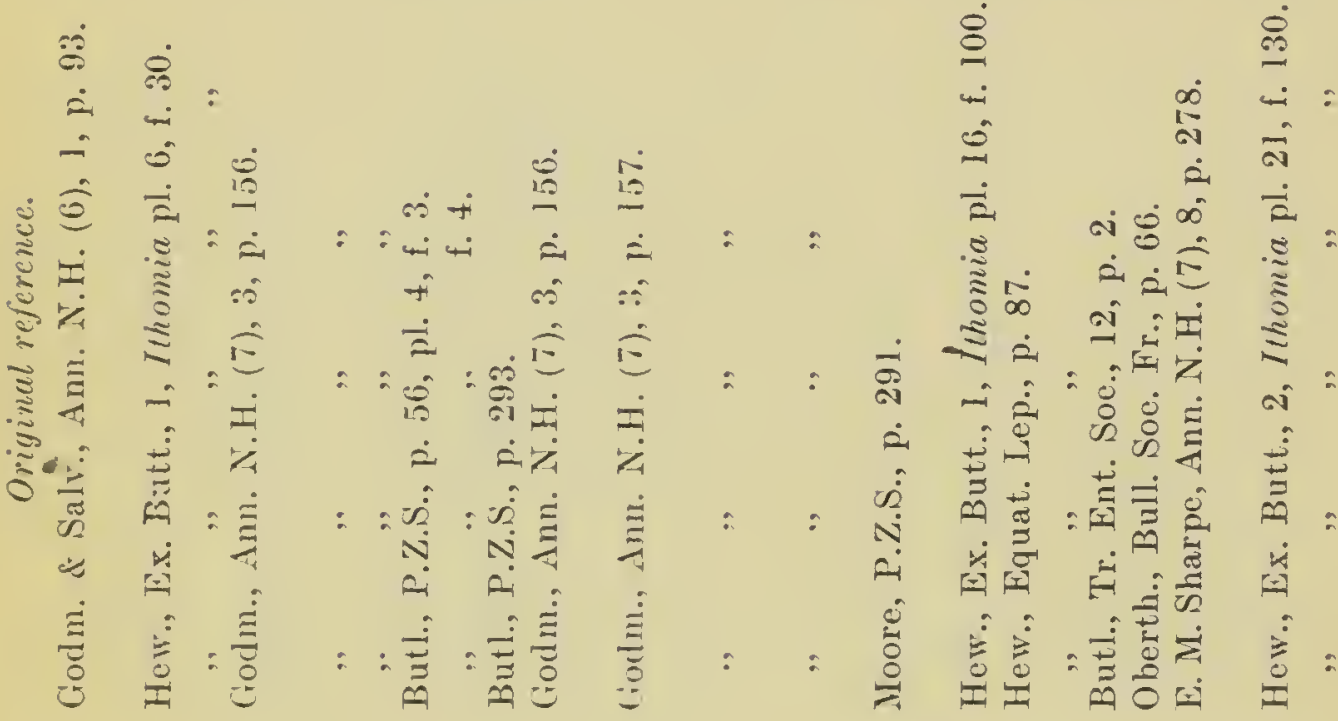

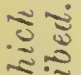

$\approx \frac{2}{3}$

$\stackrel{3}{2} \stackrel{3}{2}$

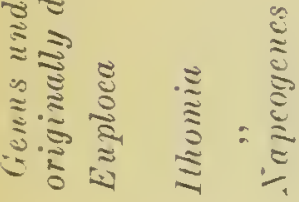

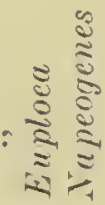

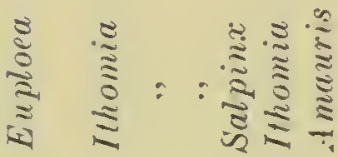

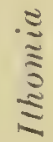

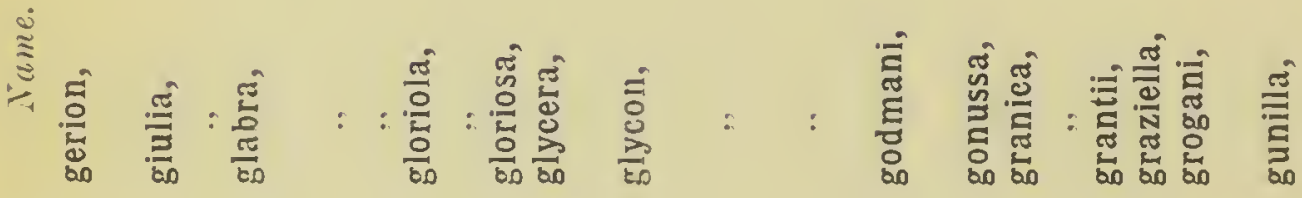

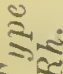

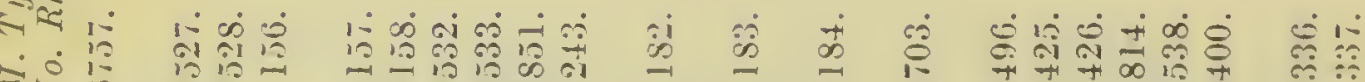

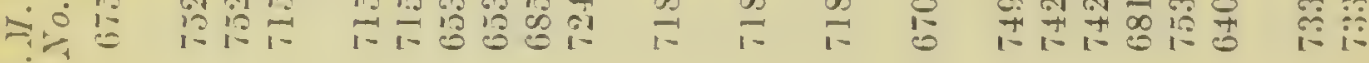




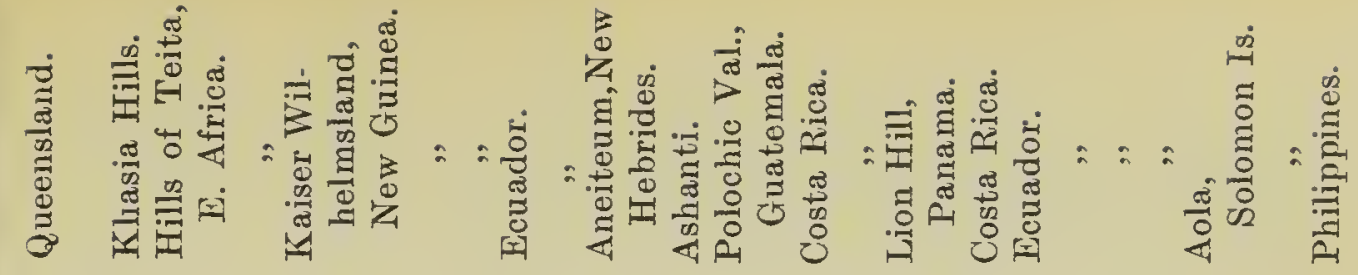

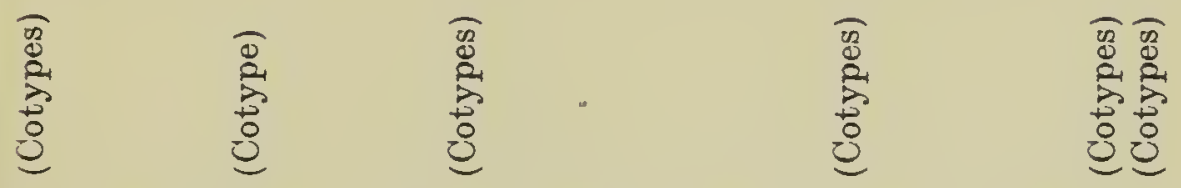

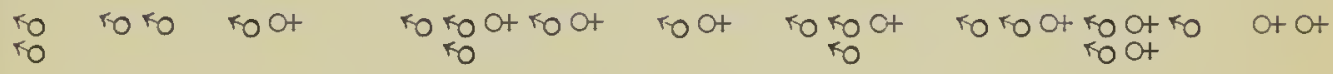

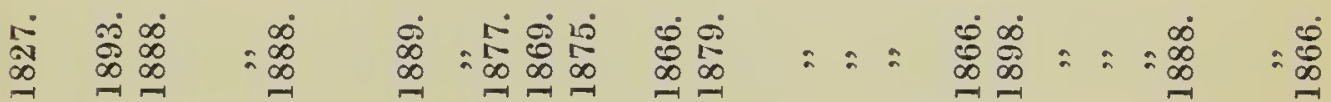

ลก 4.

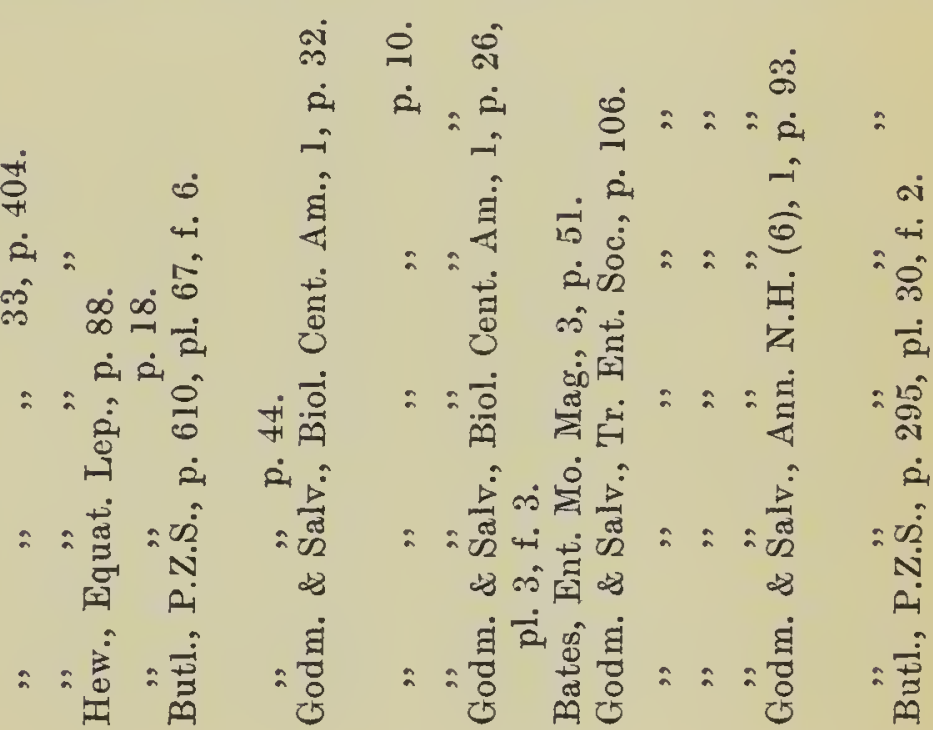

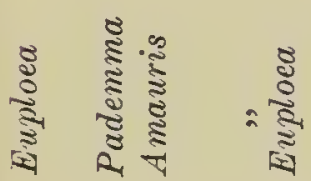

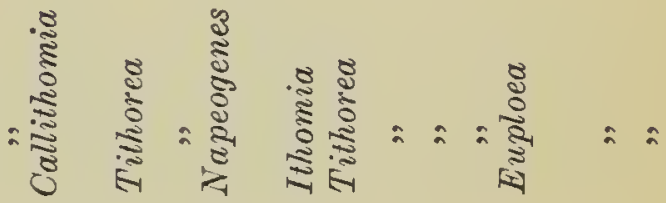

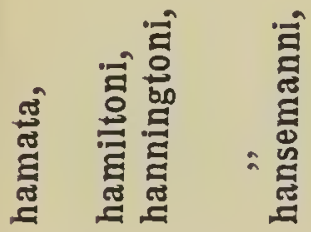

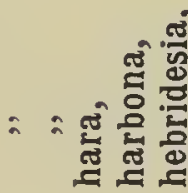

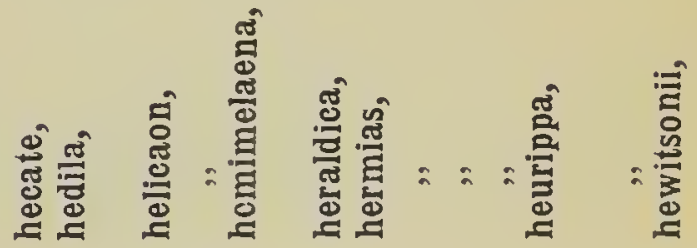

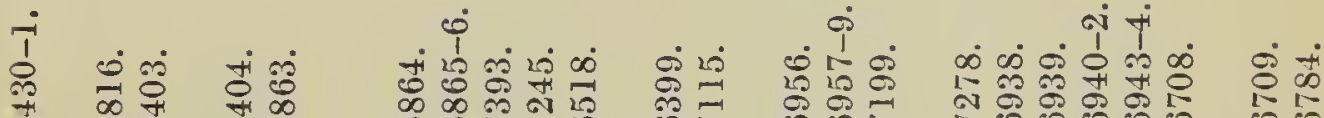

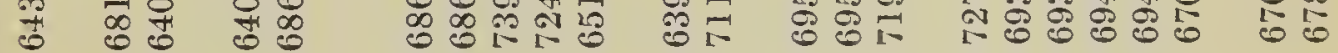




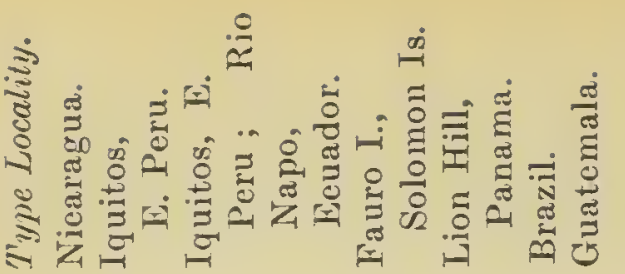

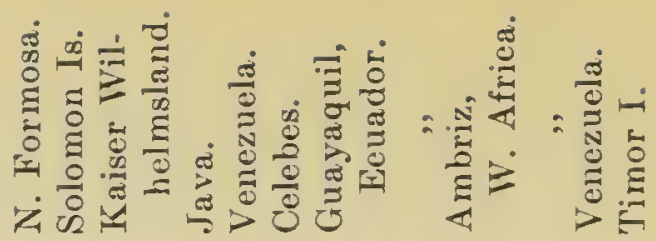

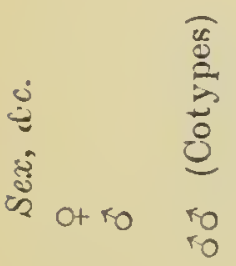

¿

ro ro otro

ro ot ot

roOt of ro

ot ro

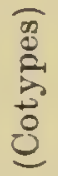

ఏ્ڤ

ro ro ro

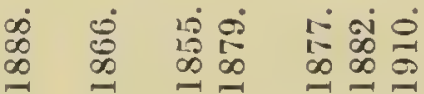

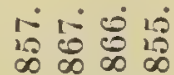

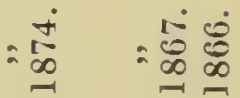

$\dot{\sim}$

is

अं क्षि

ปั

डิ

एँ

: $\approx$ i

舟

$\rightarrow$

- $\dot{0}$ वे

- $\dot{1} .8$

में के

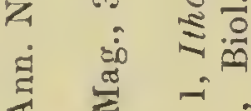

$-\infty$

$+\infty$

¿

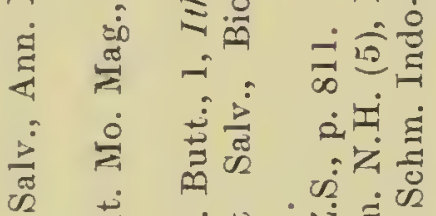

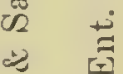

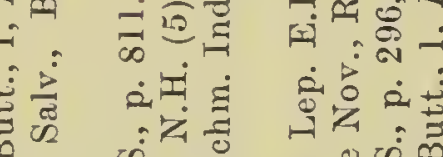

$\mapsto z$ 正

$\therefore \approx$ ¿

ठึ.

घु่ है

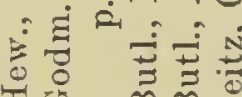

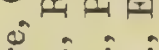

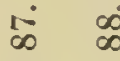

$4 \pi$ 


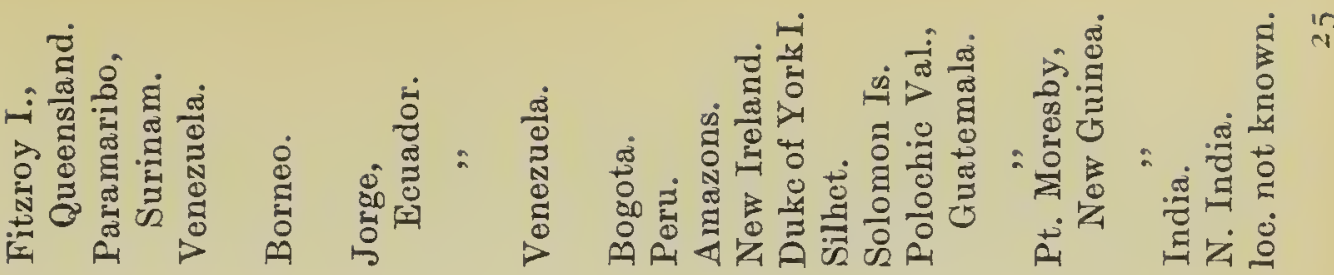

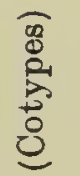

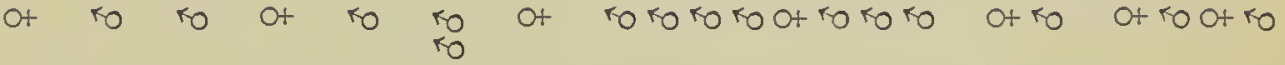

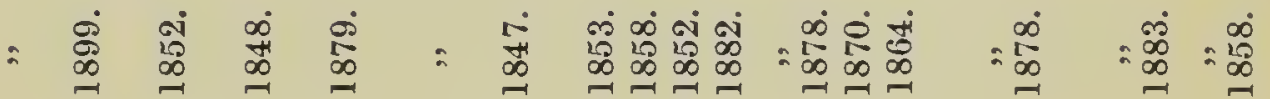

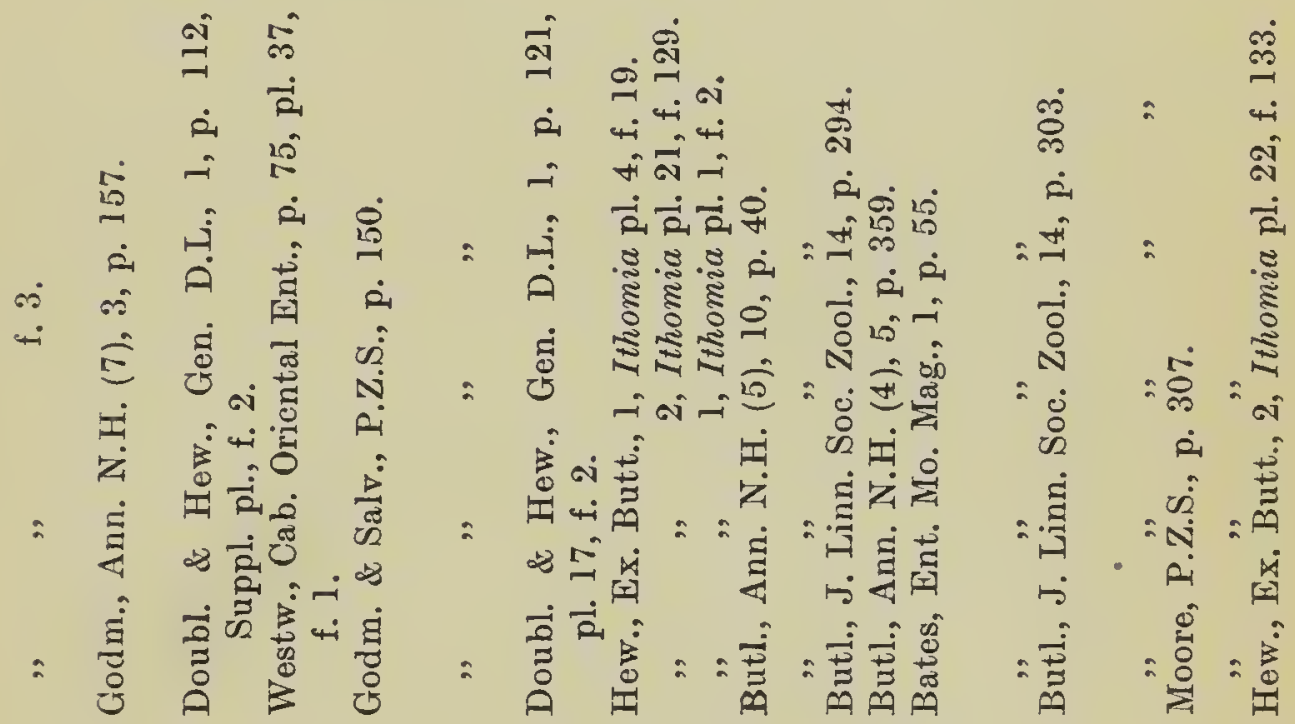

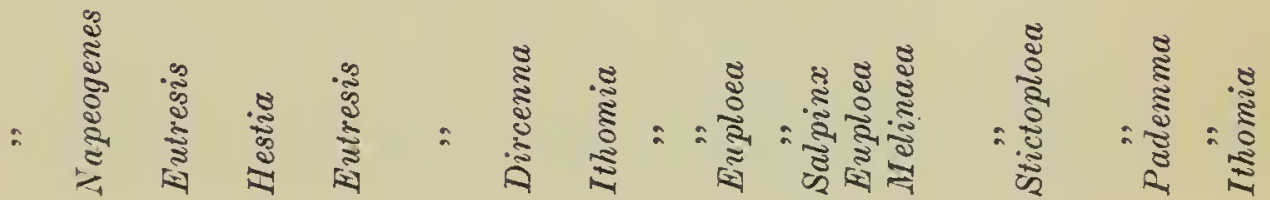

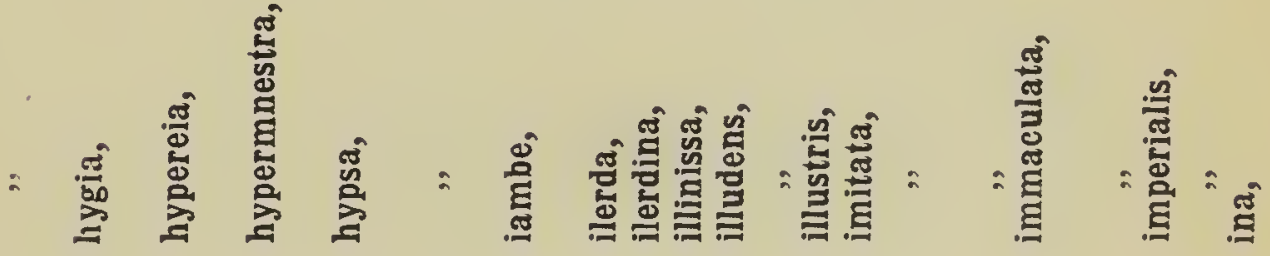

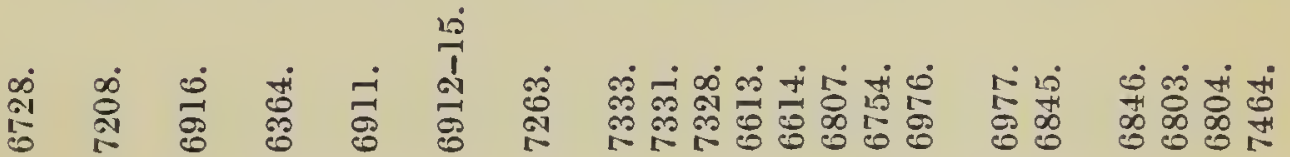




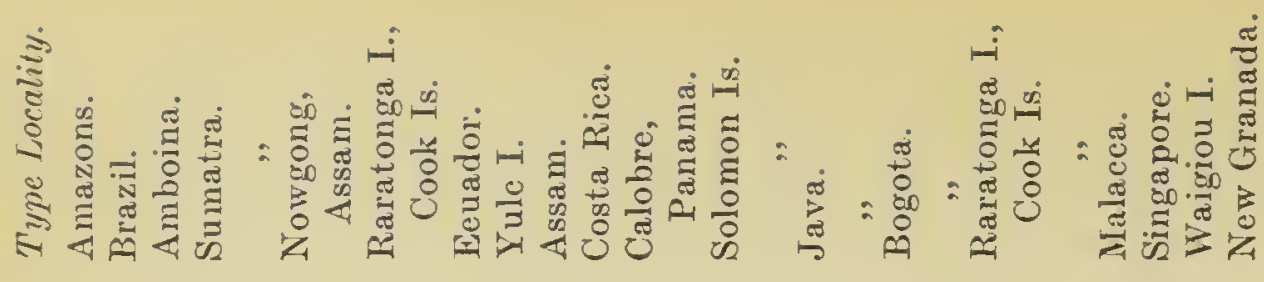

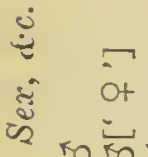

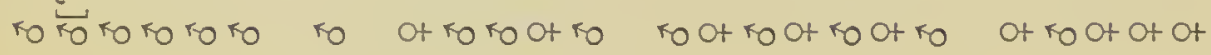

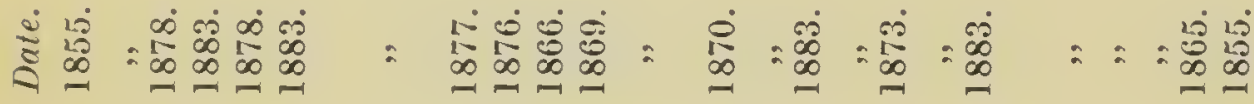

$\oplus \ddot{0}$

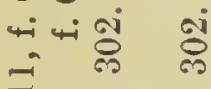

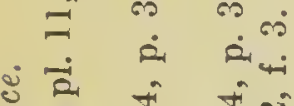

एँ

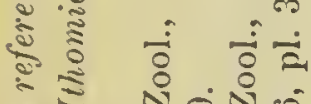

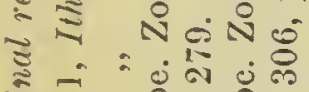

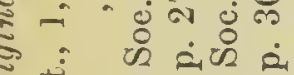

Q

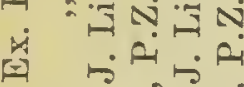

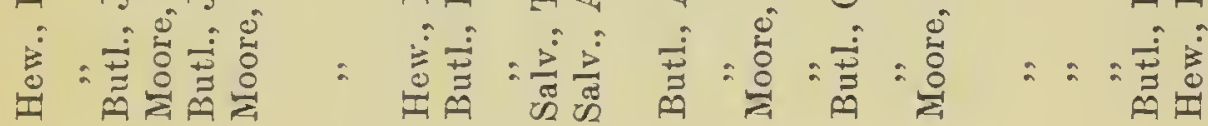

$\therefore$

4

$\stackrel{0}{0}$

की

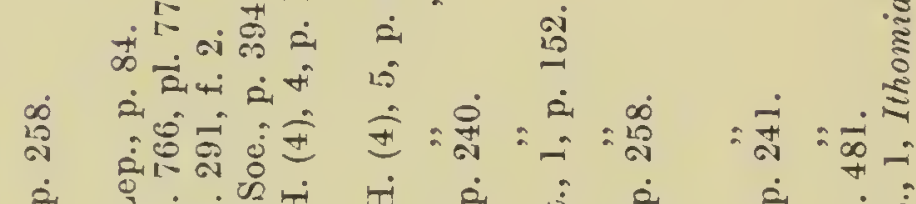

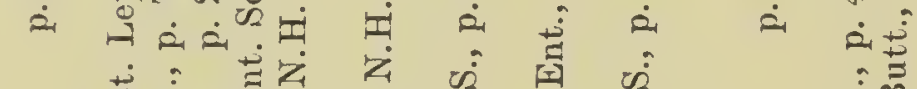

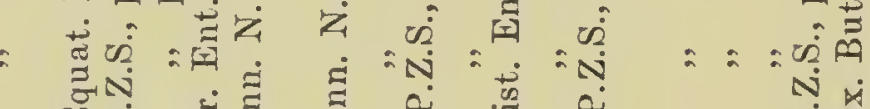

تُ

$\approx \ddot{8}$

$\frac{2}{2}: \frac{1}{2}$

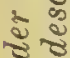

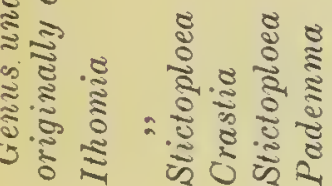

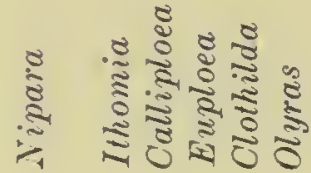

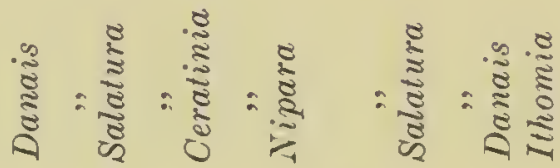

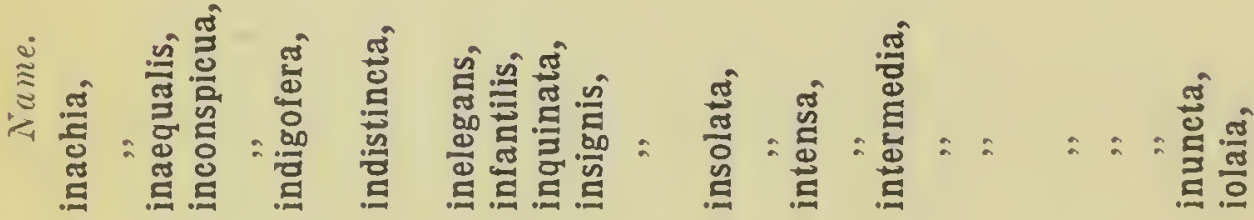

$\underset{3}{3}$

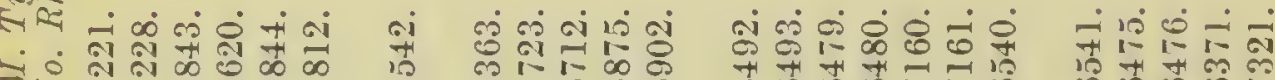
可热 


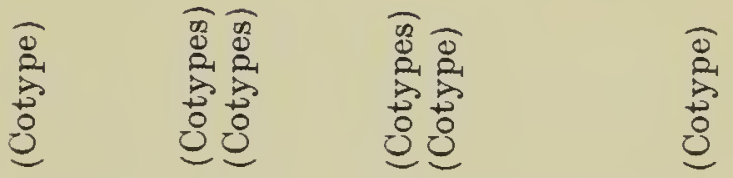

ro ot ot rootro ro root root ro root rootroot root

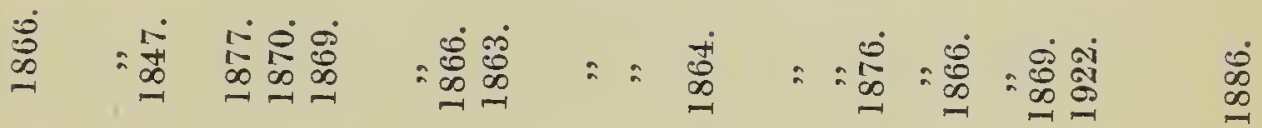

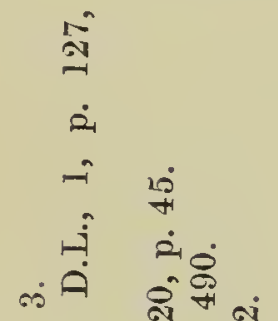

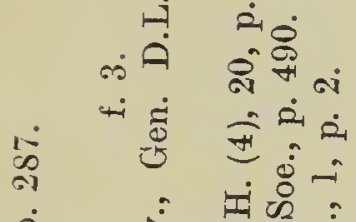

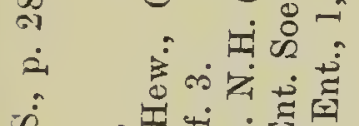

نे a

ن.

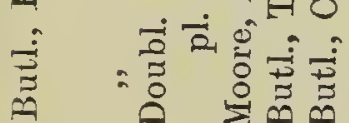

: $=$ :

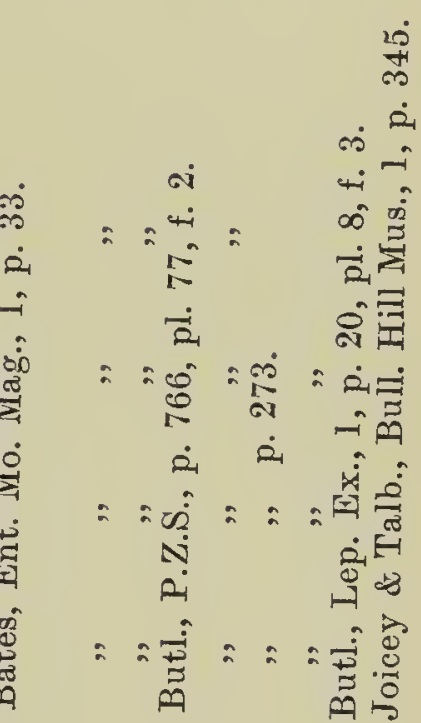

0
0
0

जi $\dot{0}$

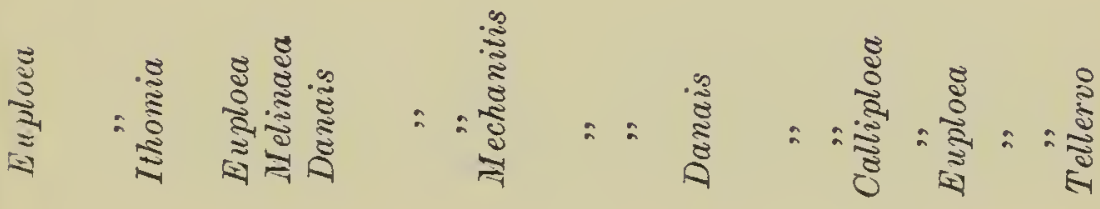

हैं

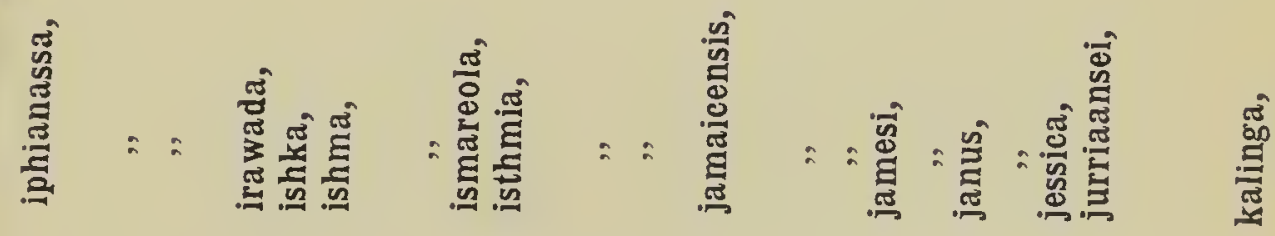

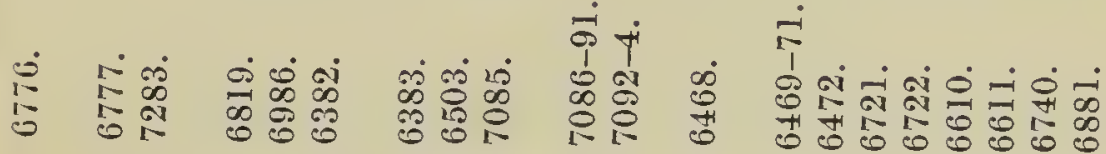




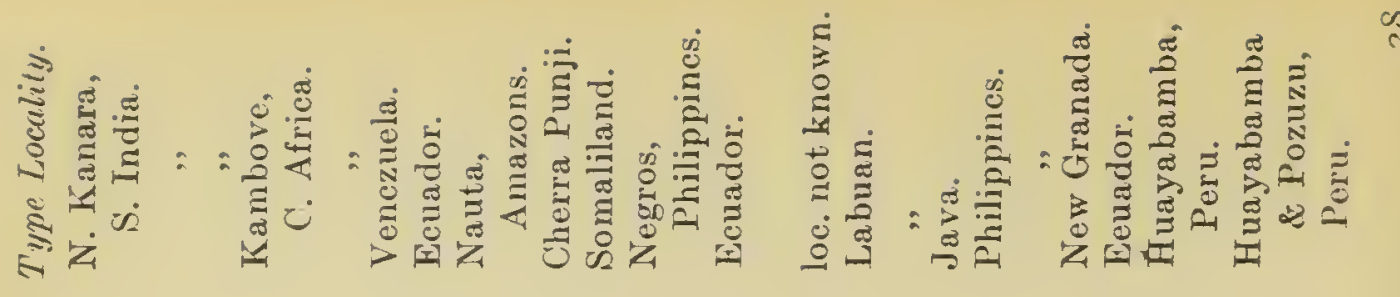

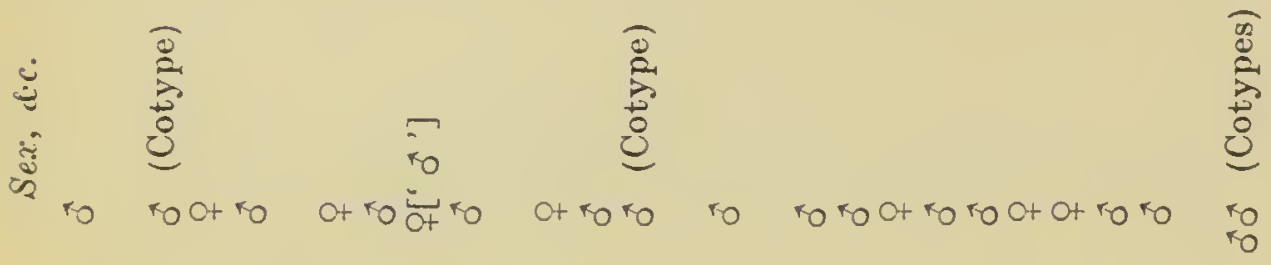

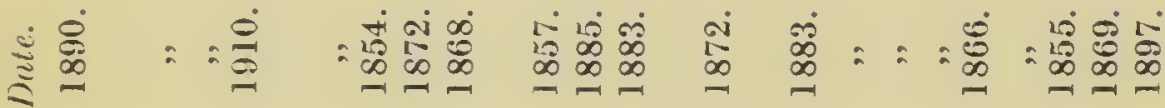

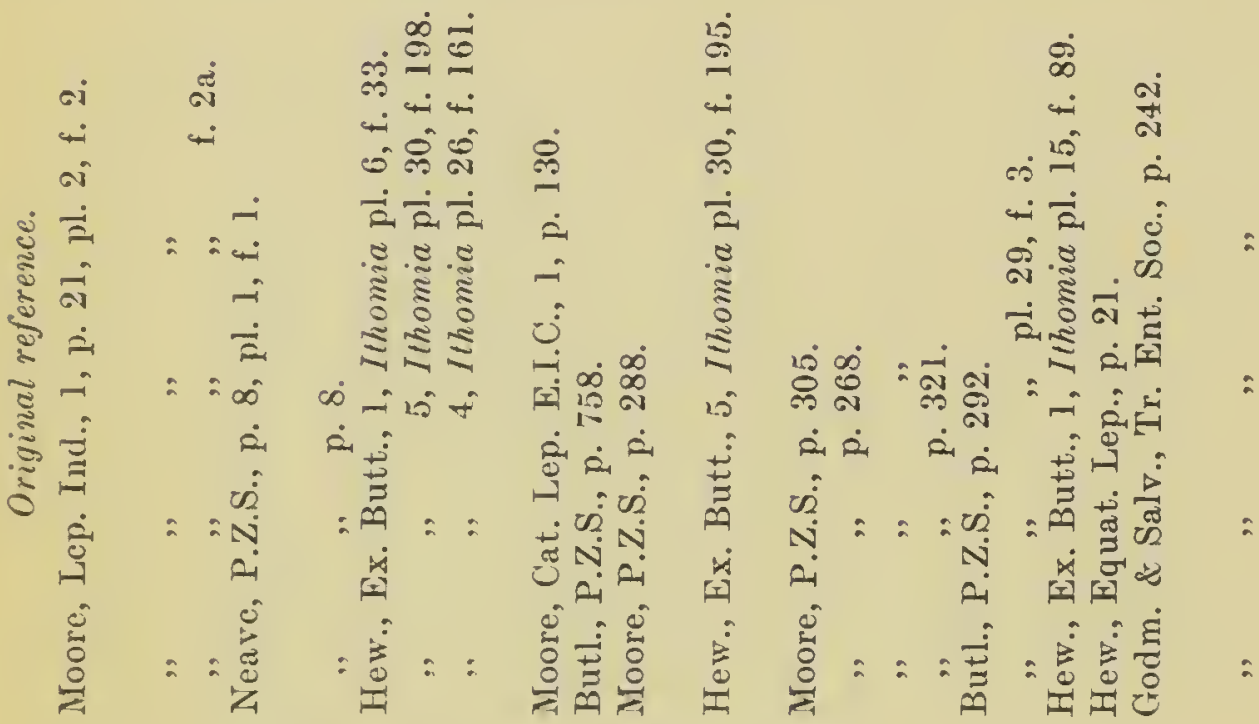

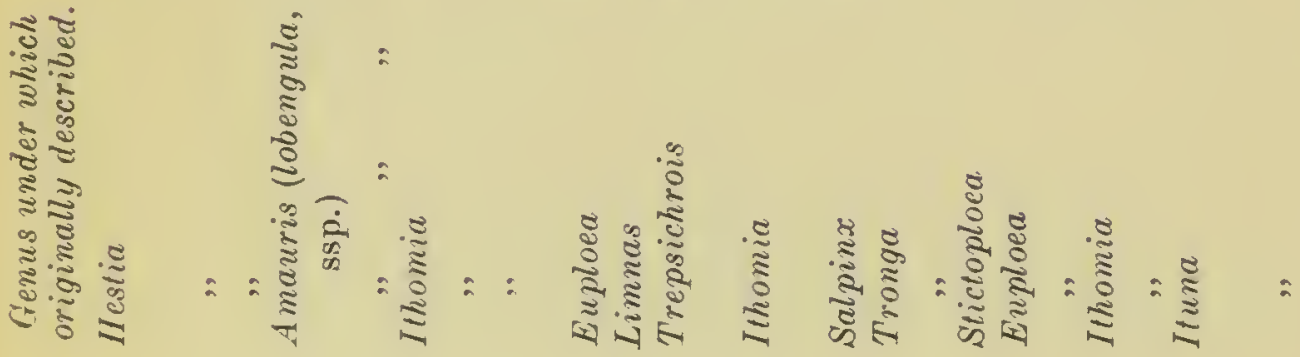

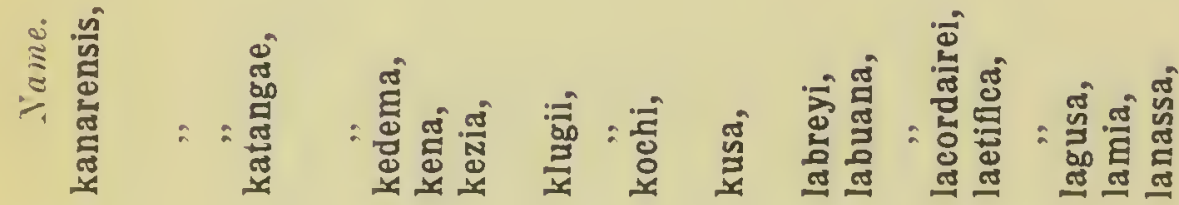

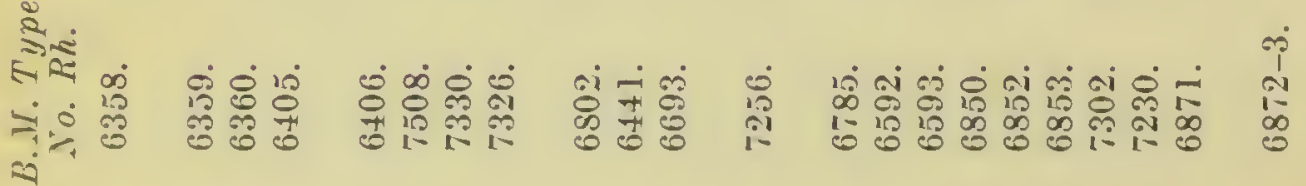




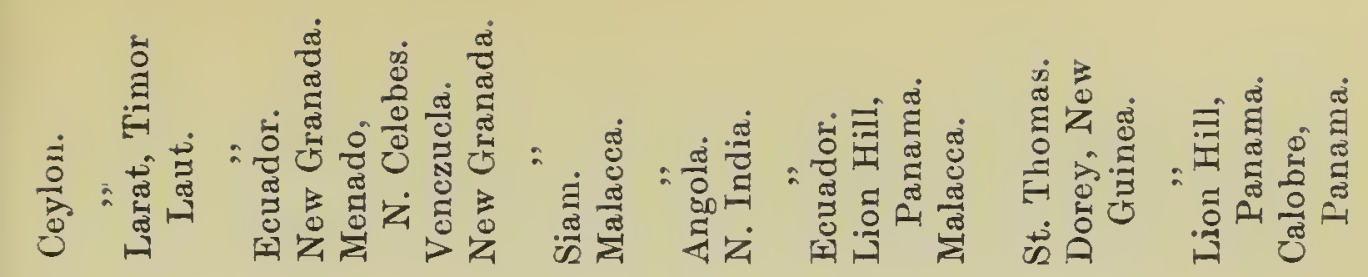

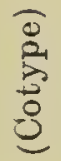

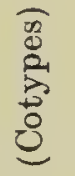

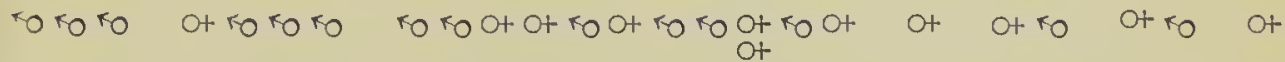

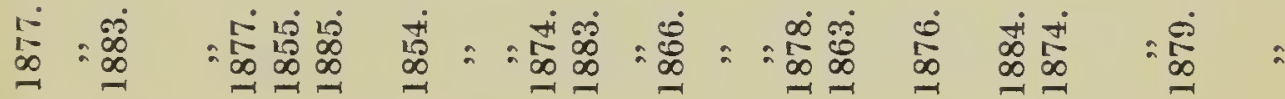

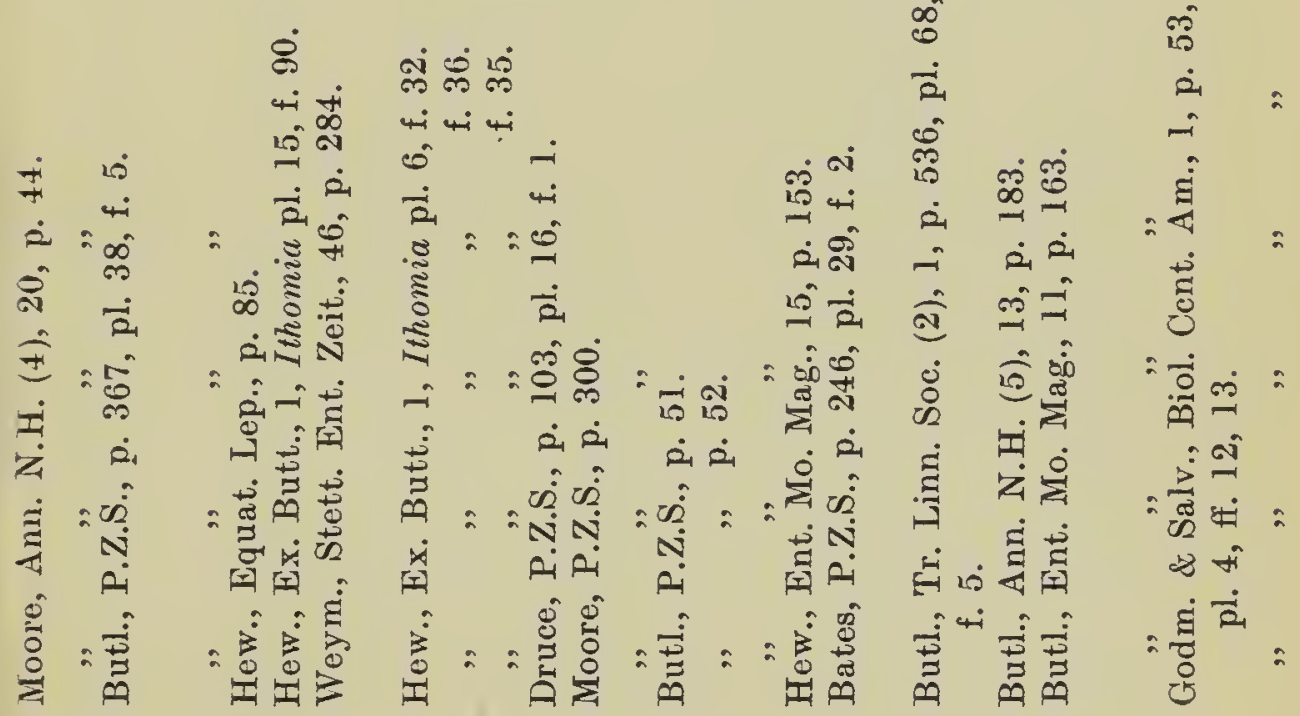

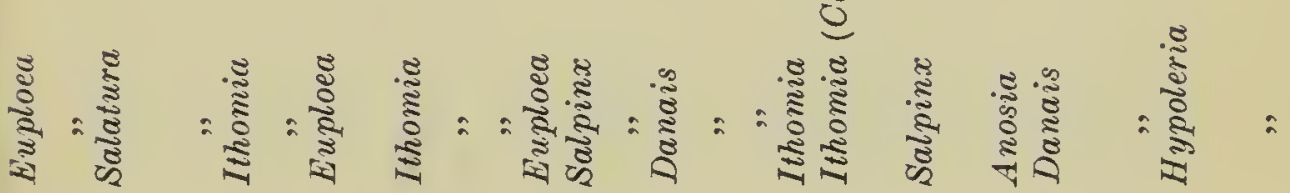

至

$\stackrel{0}{1}$

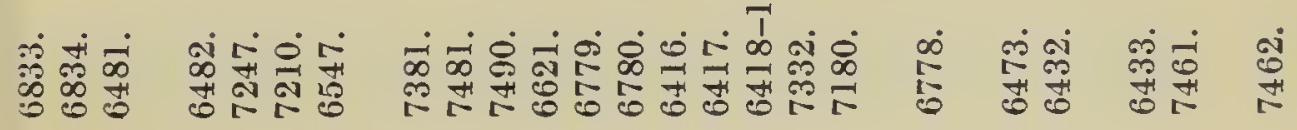




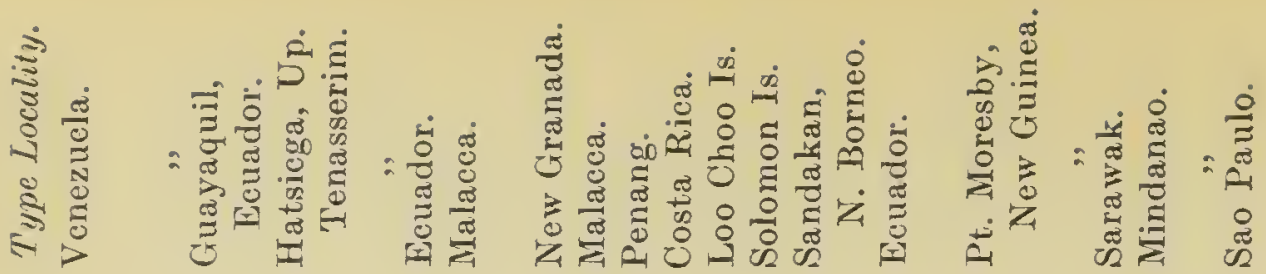

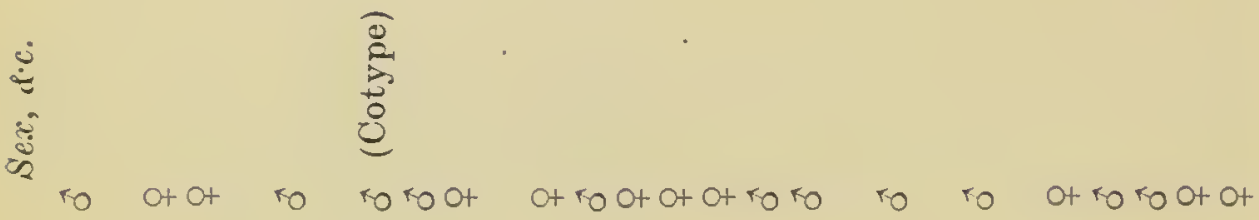

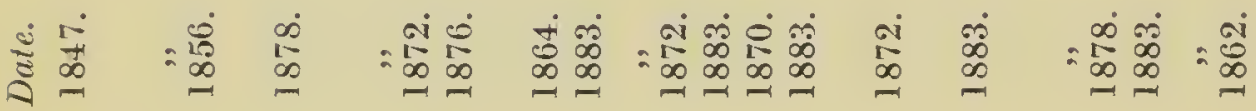

$\stackrel{\infty}{0}$

$\therefore \infty^{\infty}$

क्ष

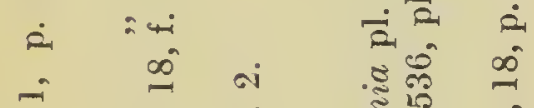

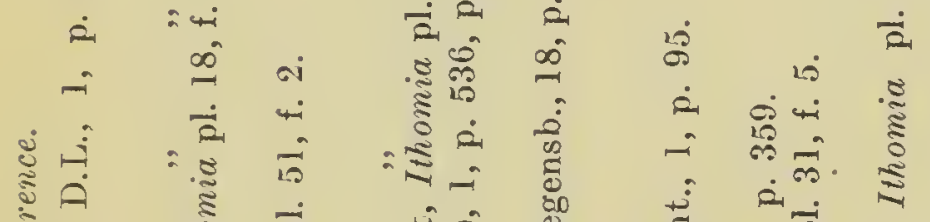

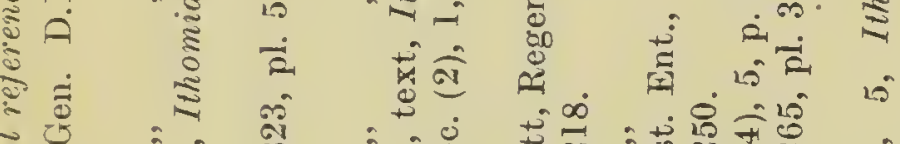

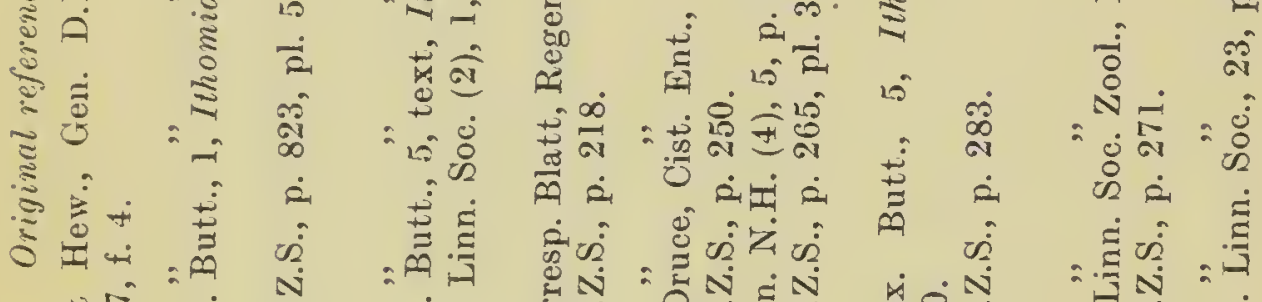

\#

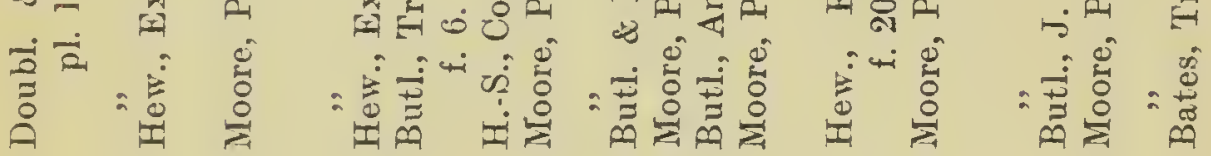

ก ับ

इ.)

ఫे

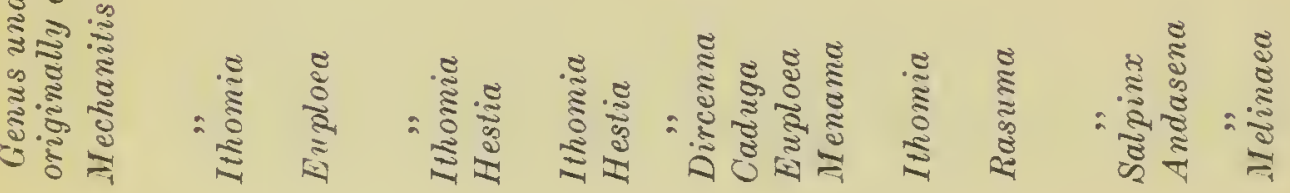

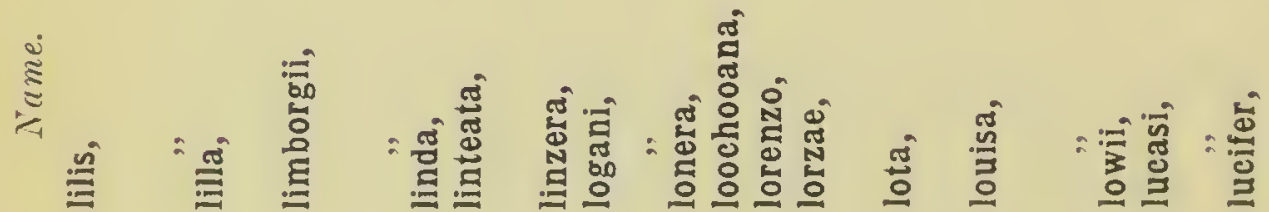

\&ิ

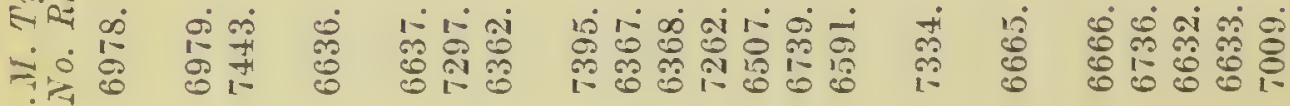




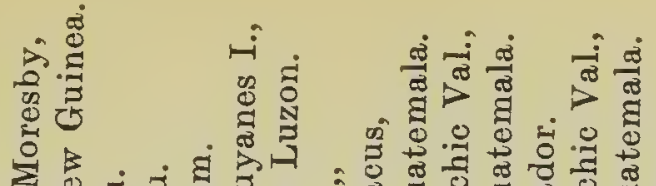

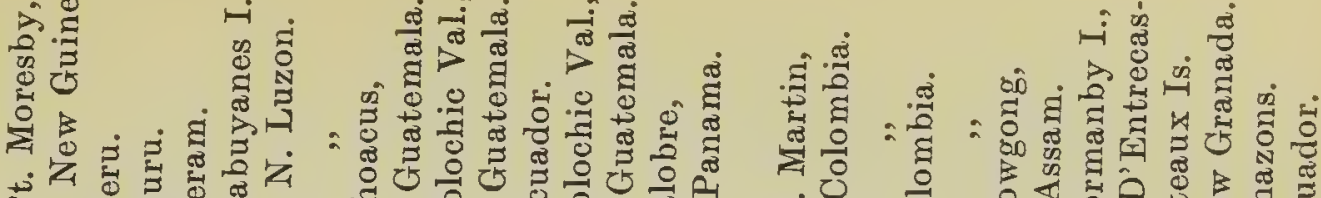

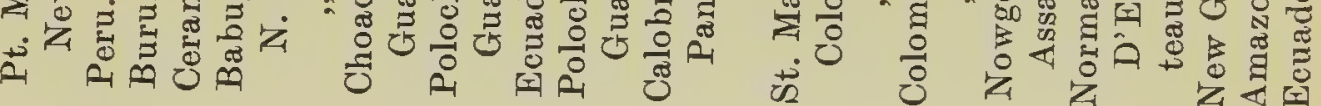

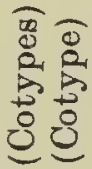

ro otrootro otro ot roro ot ro otroot ot rot roto

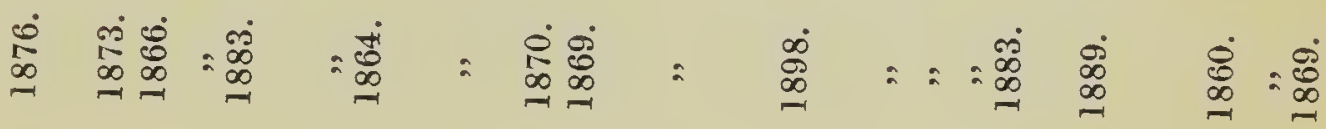

ลี่

i.

$\stackrel{\text { in }}{\rightarrow} \stackrel{\infty}{=}$

$\stackrel{i}{\rightarrow} \quad \stackrel{8}{0} \dot{0}$

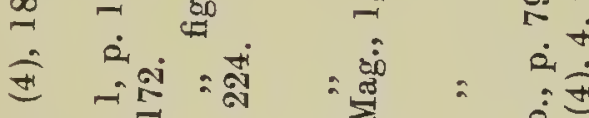

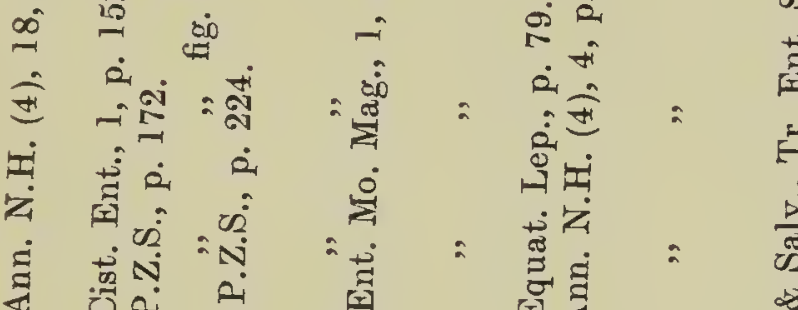

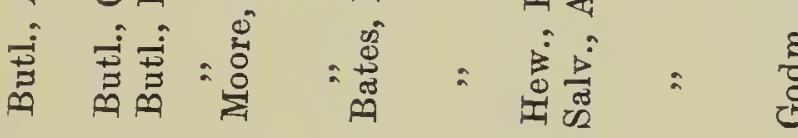

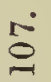

$\pm \therefore$

$+i$

$\therefore \quad+\quad \dot{0}$ के

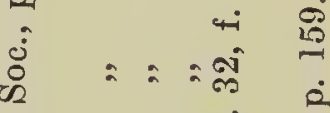

承

i. $\overrightarrow{2}$

$\stackrel{\approx}{\approx}:$

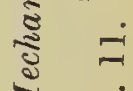

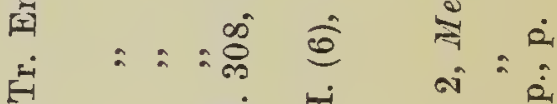

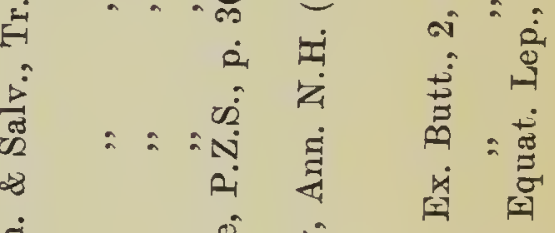

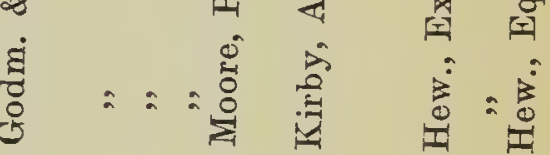

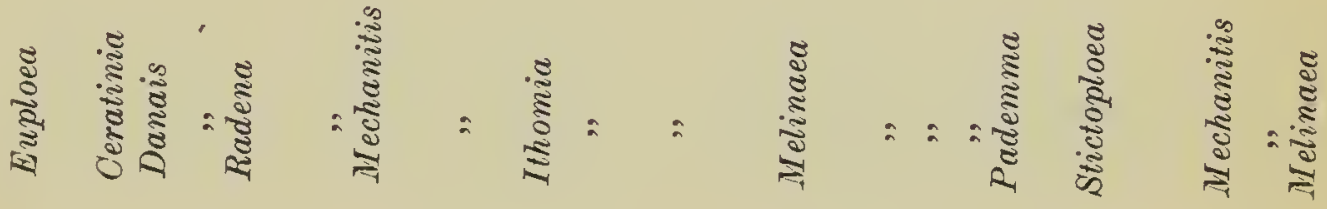

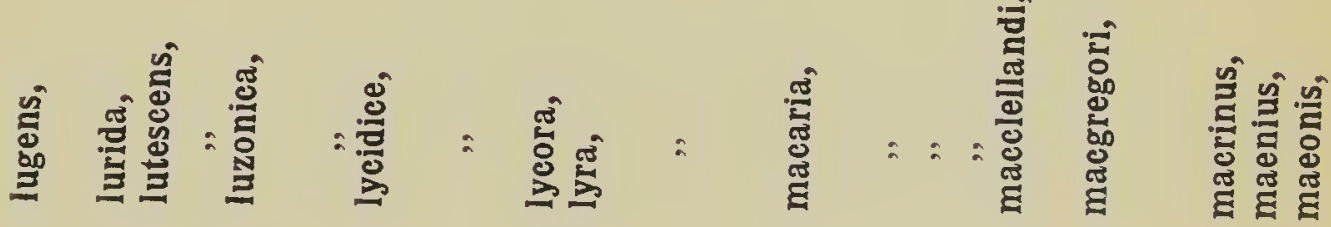

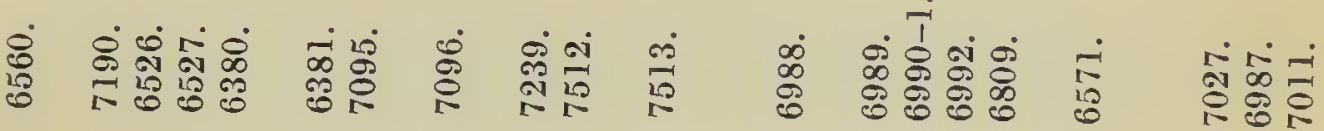




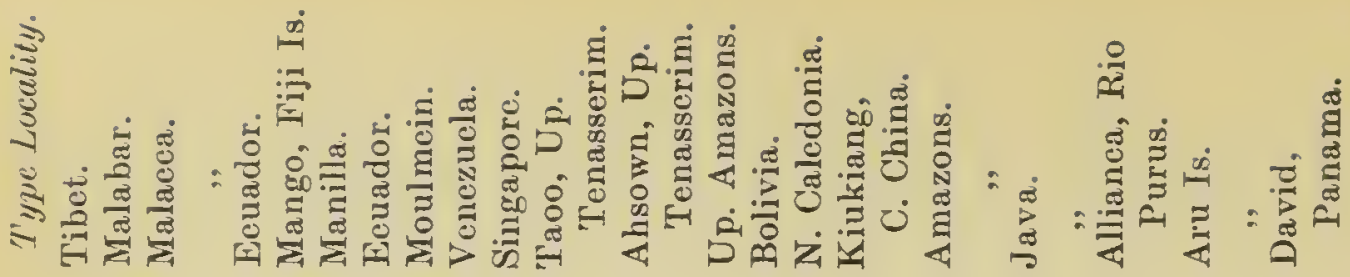

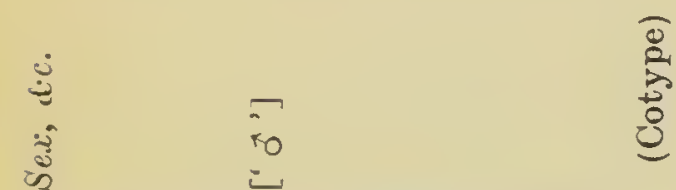

ot ot root of otroromotroro ro otototro rootrootro rootot

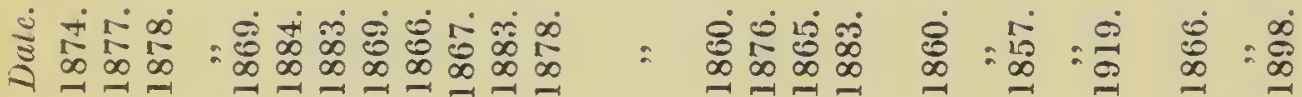

จำ $\quad \dot{0} \infty$

-

$\stackrel{5}{a+1}$

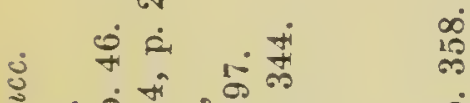

लिपं के

हंध

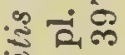

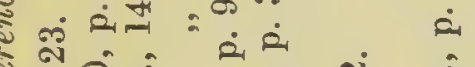

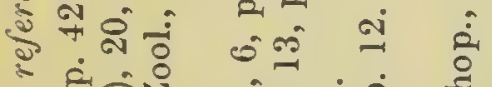

चั

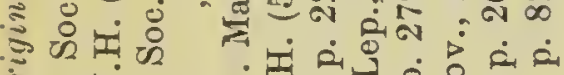

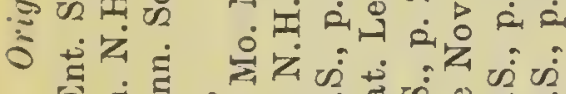

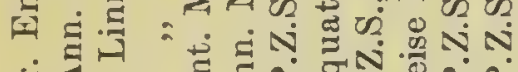

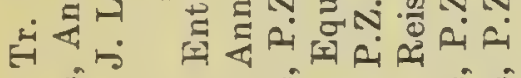

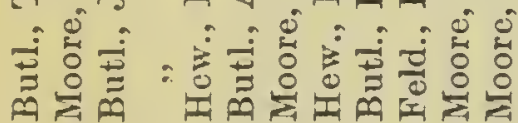

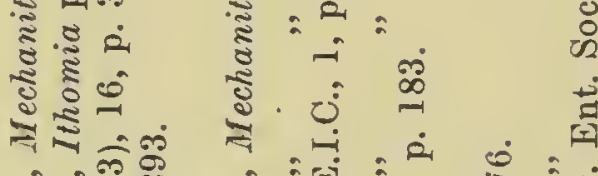

:

$\exists$

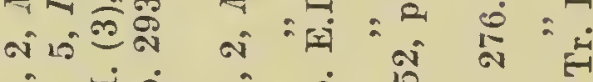

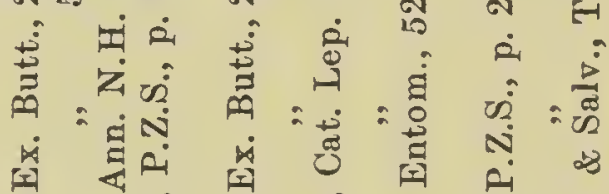

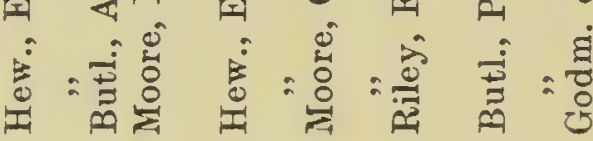

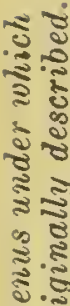

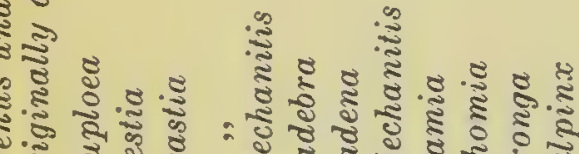

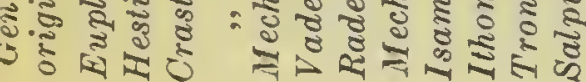

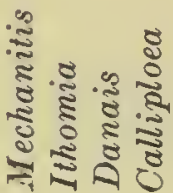
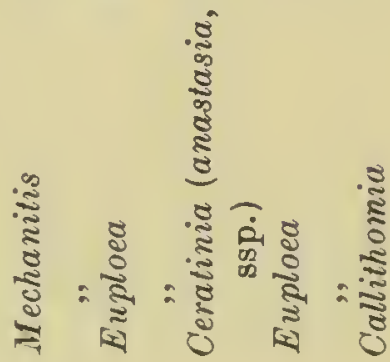

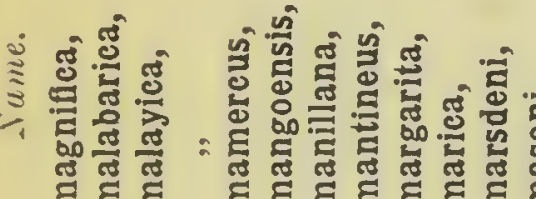

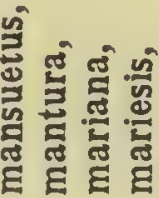

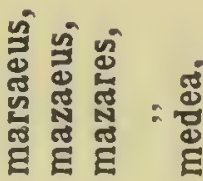

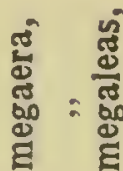

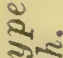

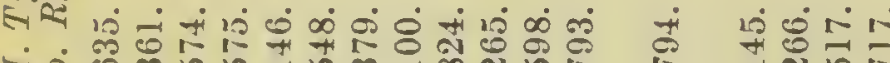
च。 
离

\&.

을

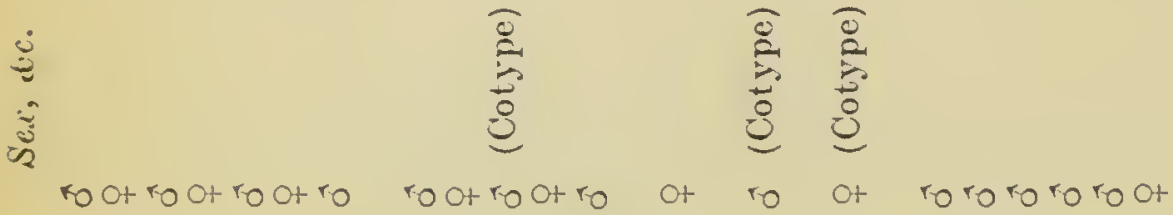

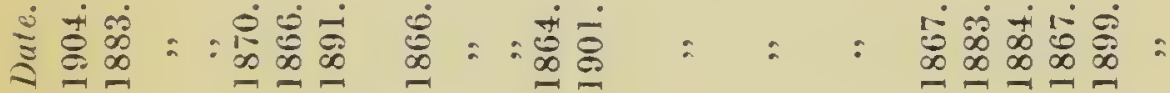

至

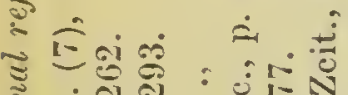

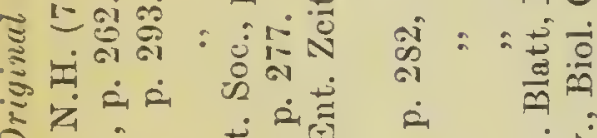

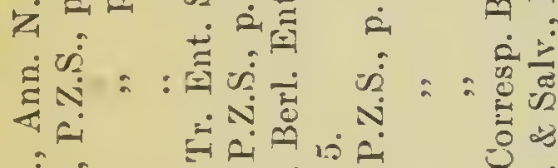

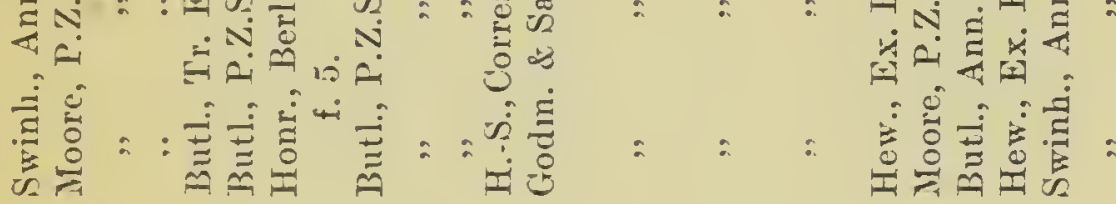

เே

- 4

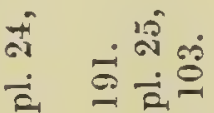

$\ddot{2} \dot{2} \ddot{2}:$

ङ

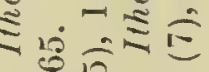

+

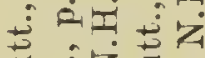

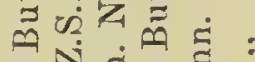

כ)

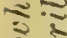

ปे

$\approx$

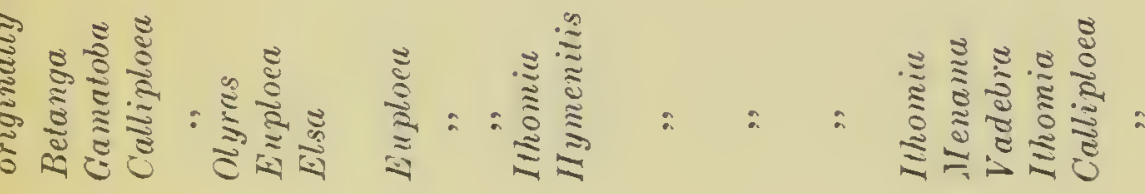

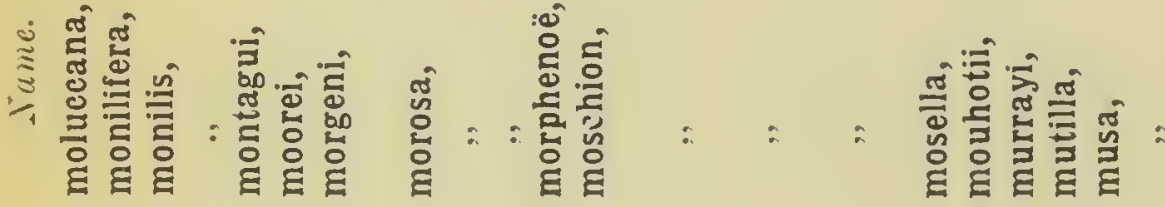

$\cong$

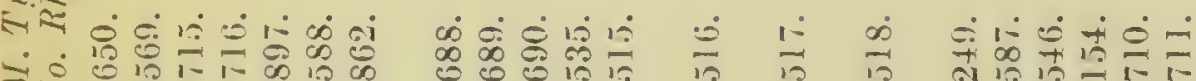

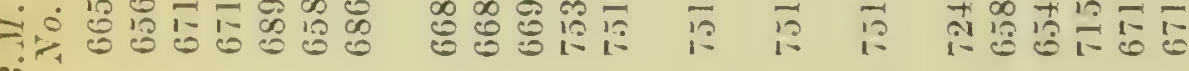
$\infty$ 


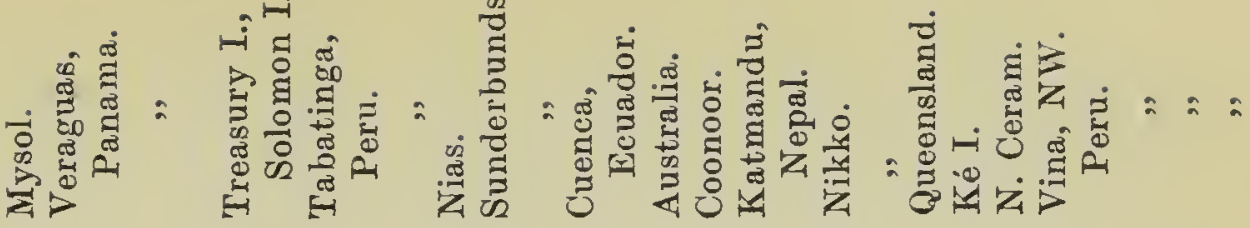

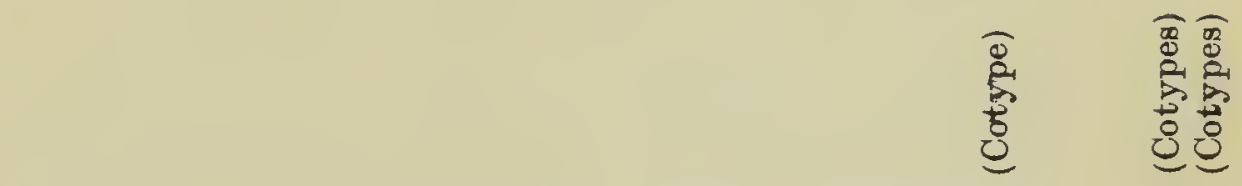

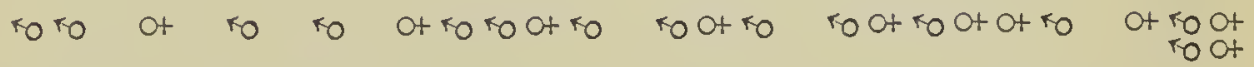

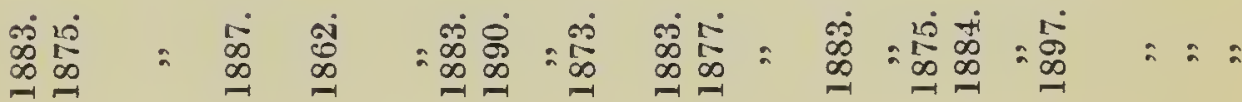

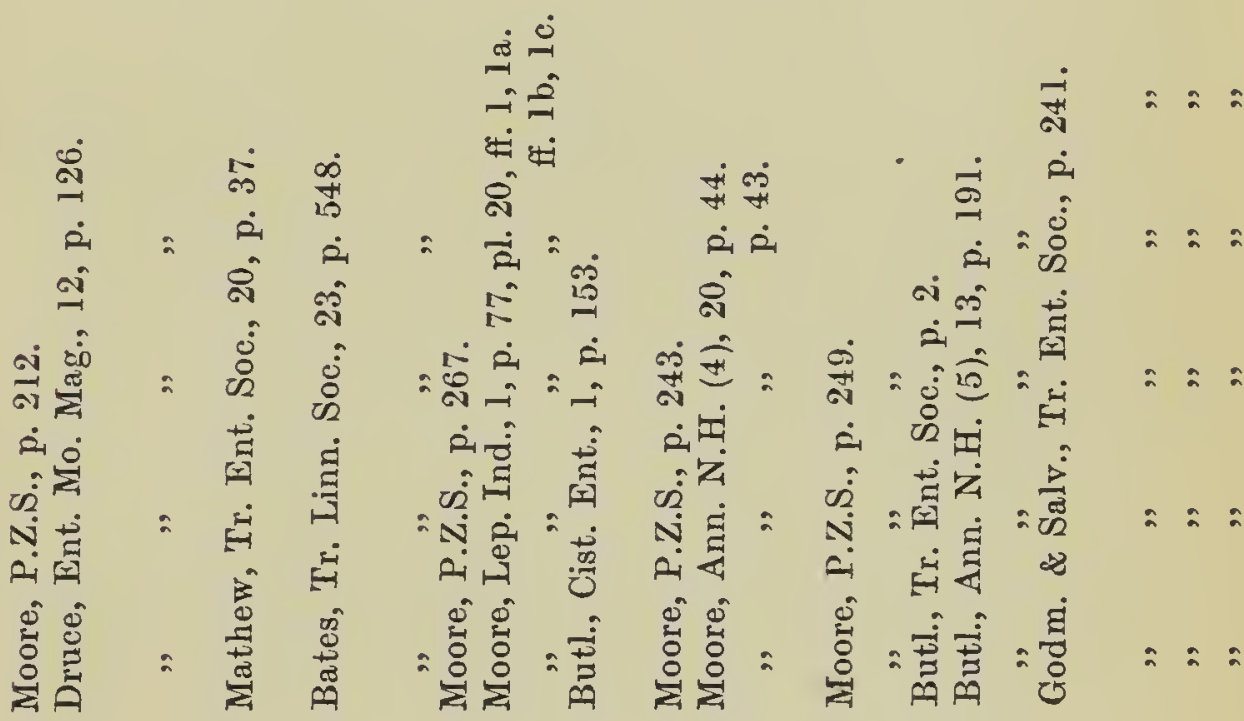

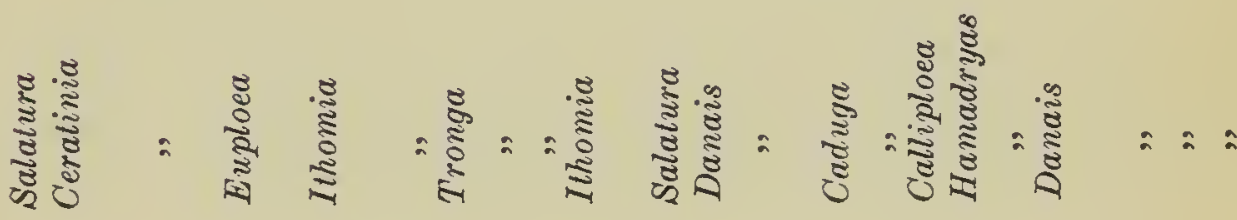

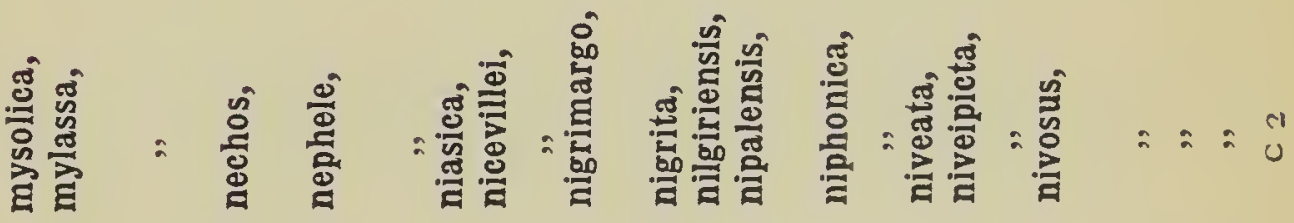

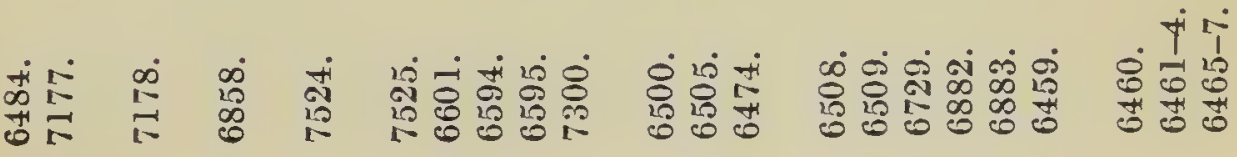




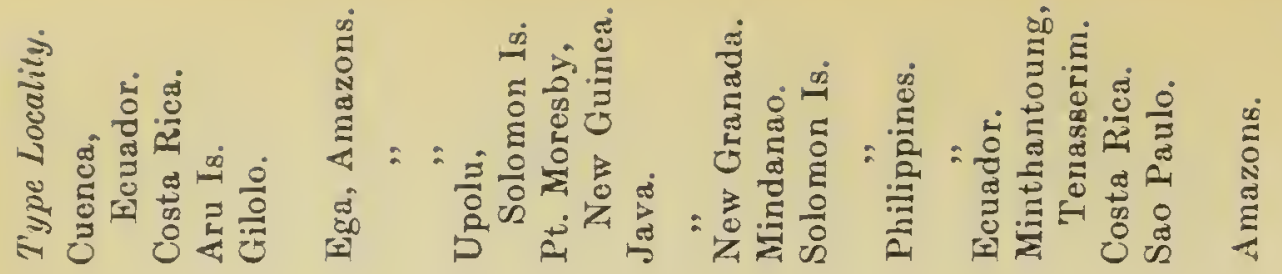

$\stackrel{\leftrightarrow}{m}$

ثัن

ฆัป

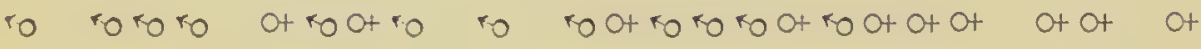

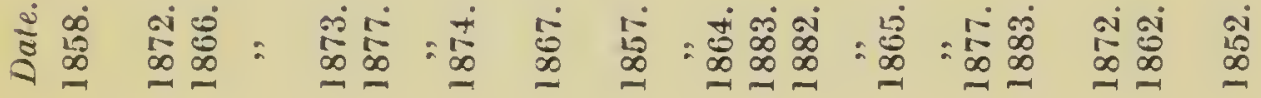

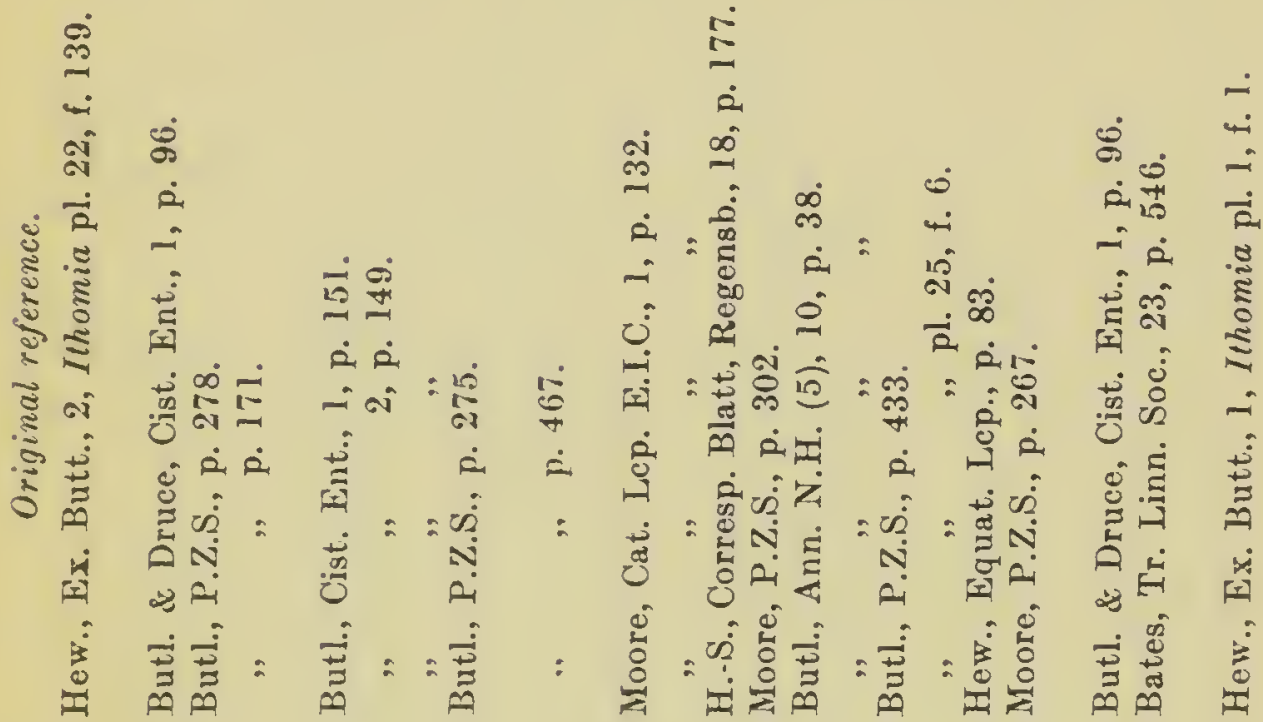

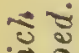

इ

ఖ

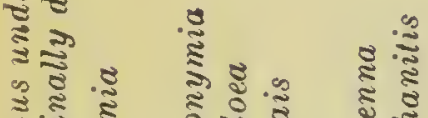

సี.

रूखे

यू

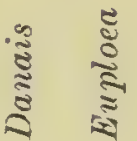

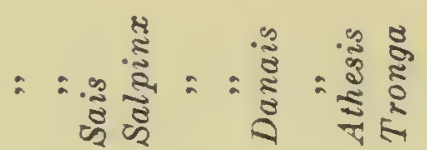

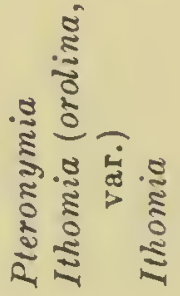

है

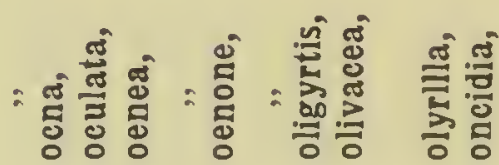

कू

$\underset{3,2}{2}$

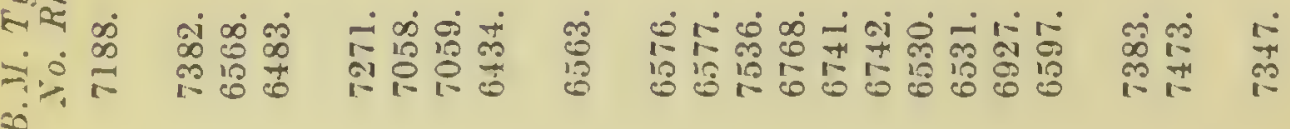




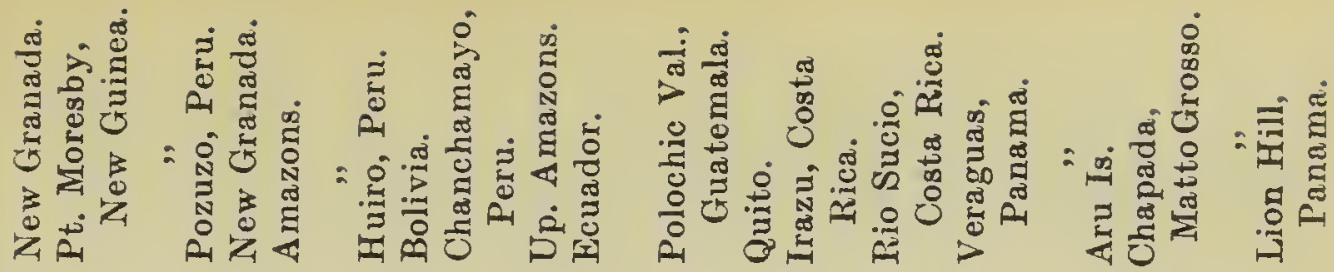

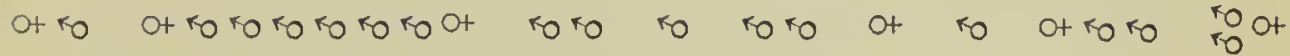

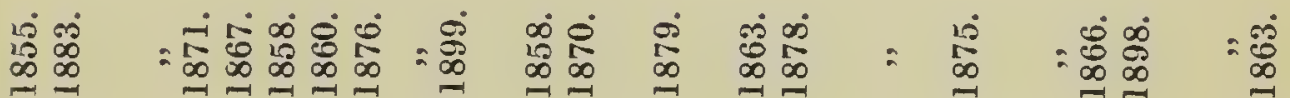

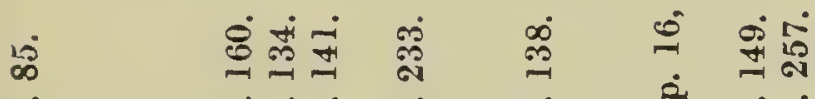

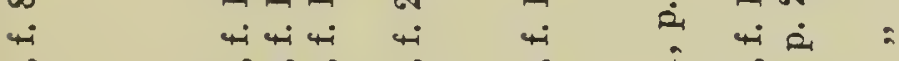

से $10^{\circ}$ สิ

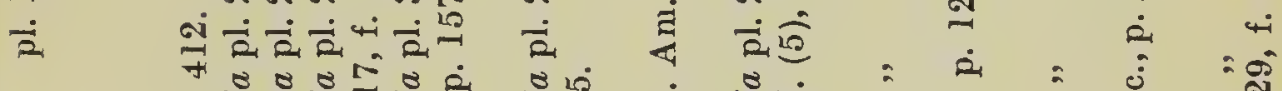

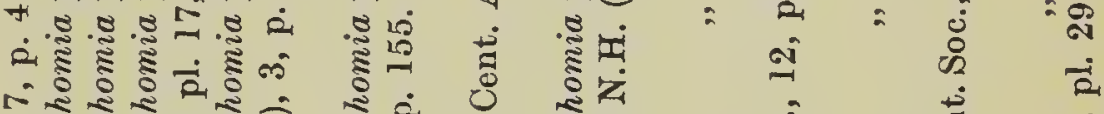

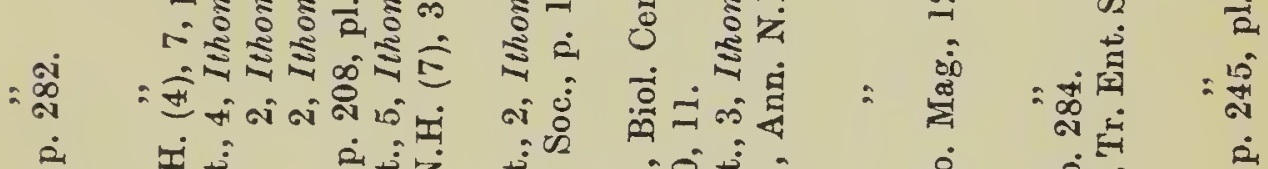

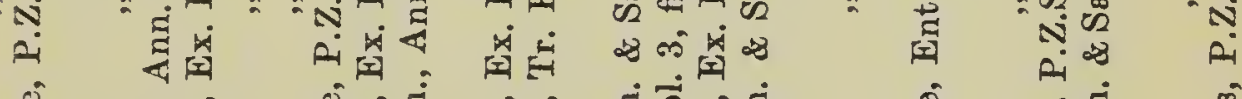

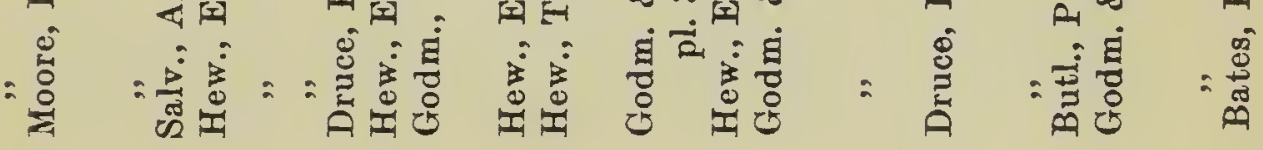

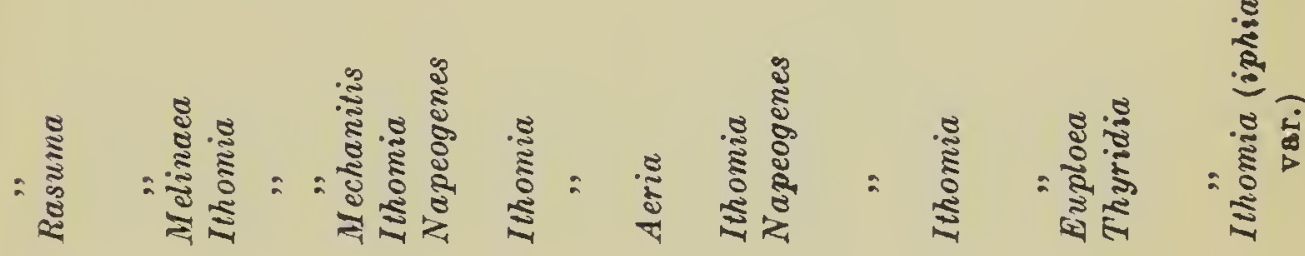

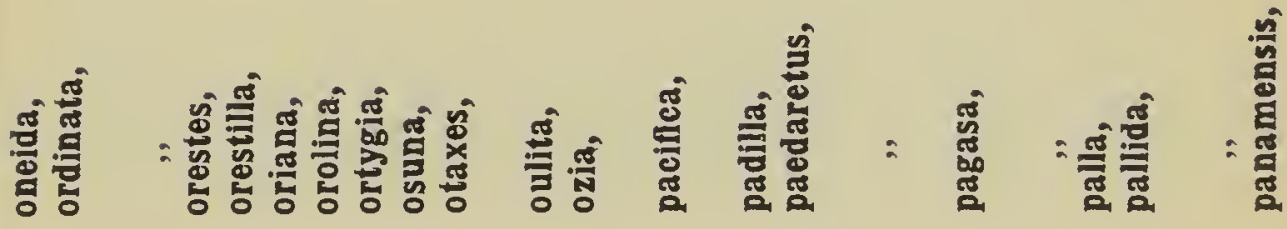

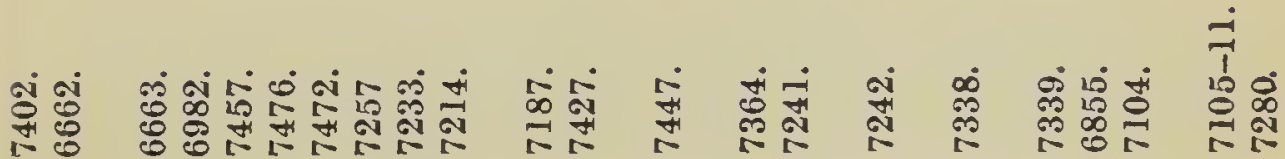


离 हैं

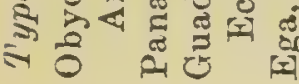

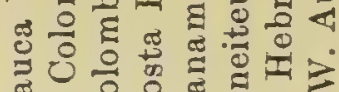

गु

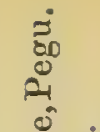

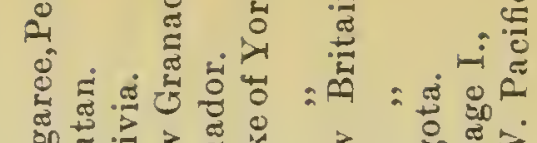

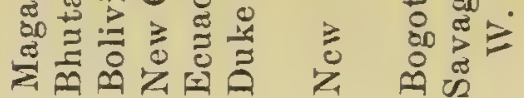

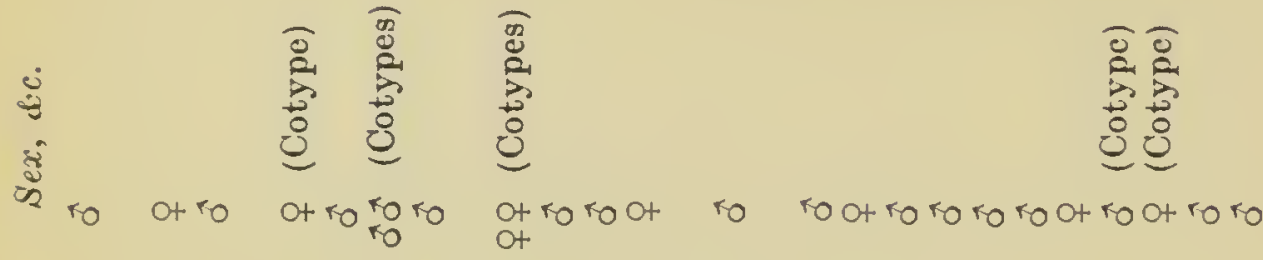

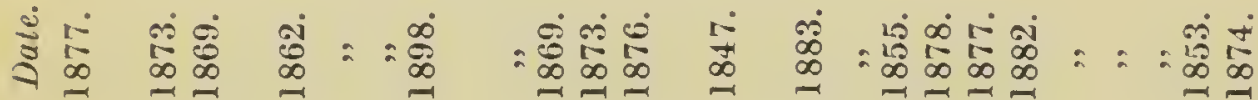

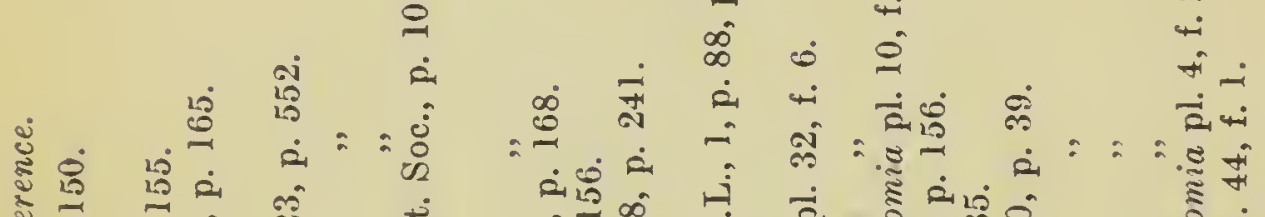

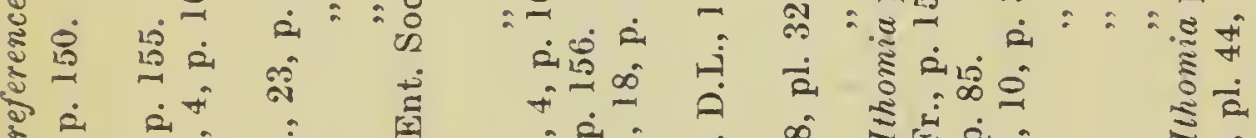
范 志部

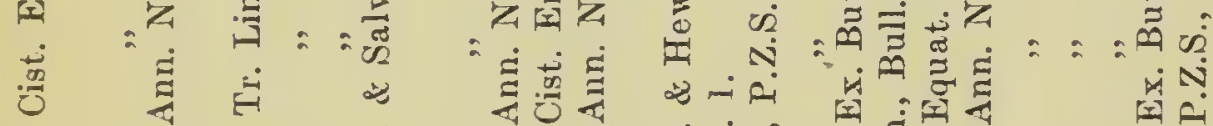

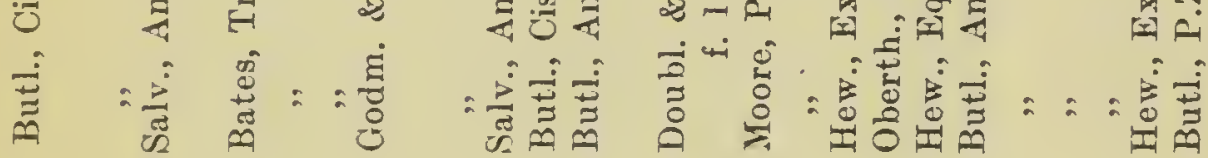

ปู่

इี है

ఫั

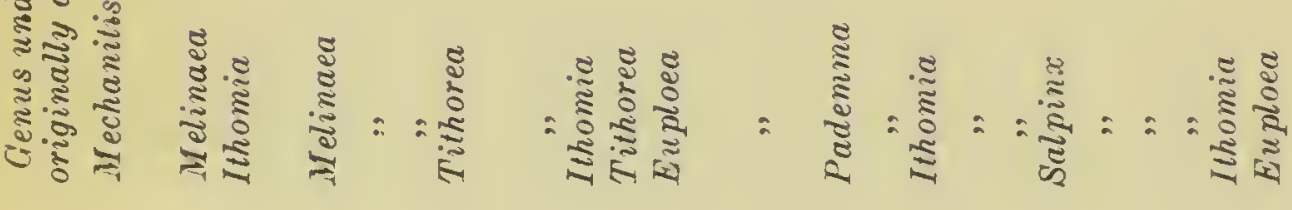

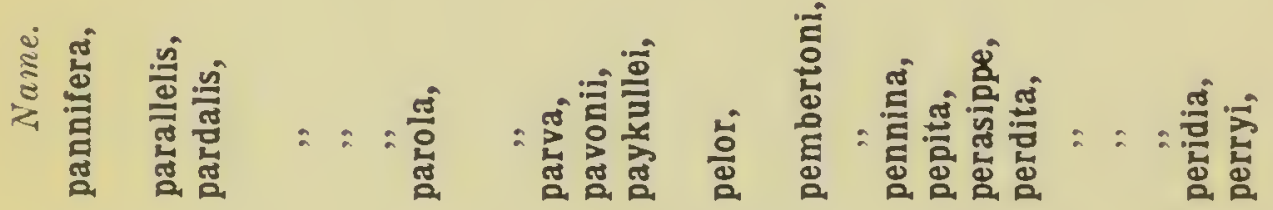

ณี่

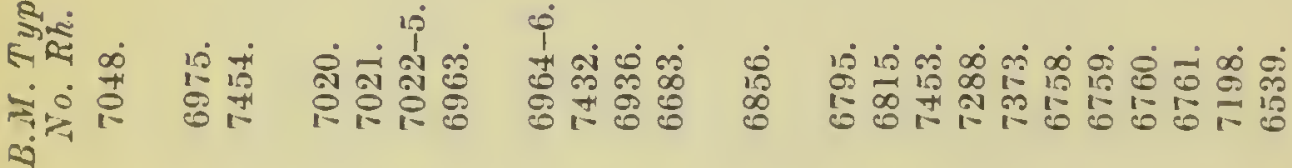




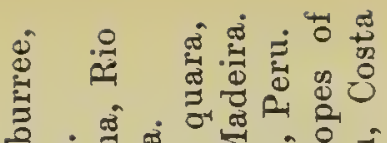

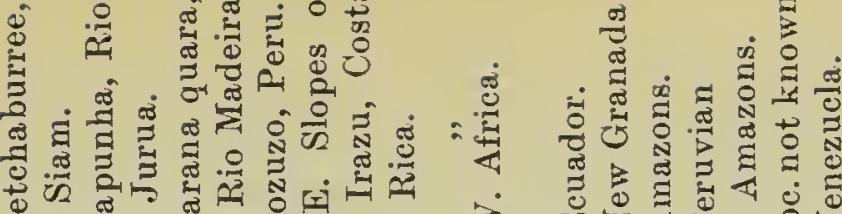

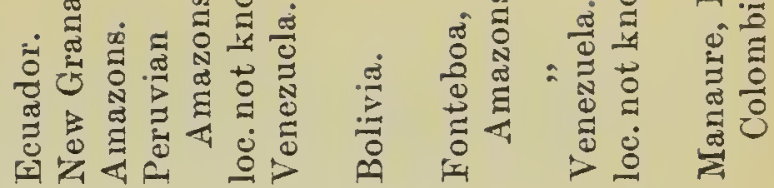

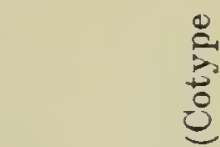

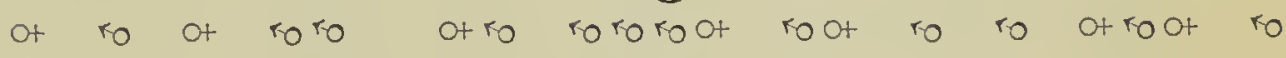

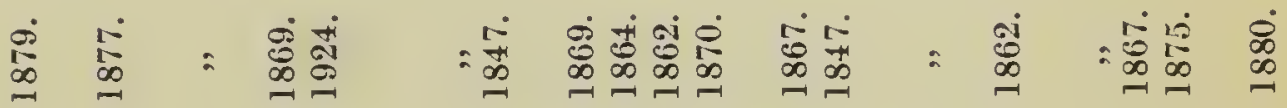

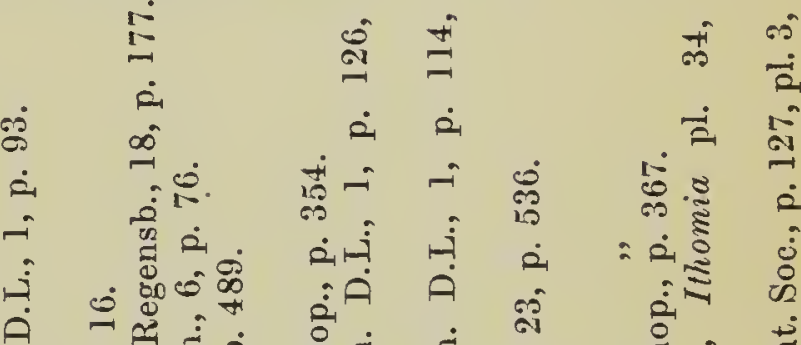

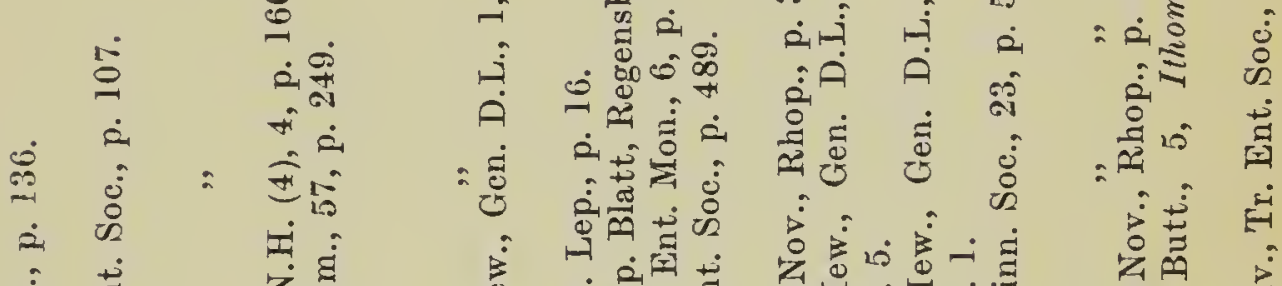

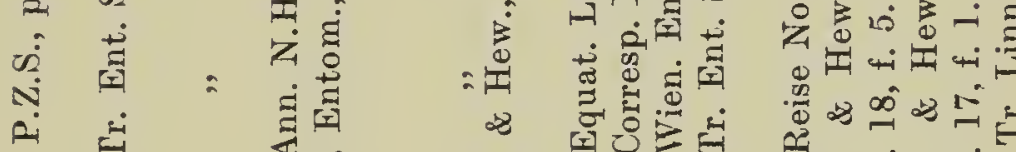

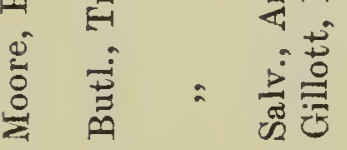

\&

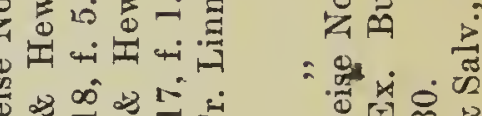

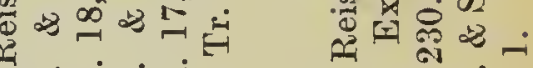

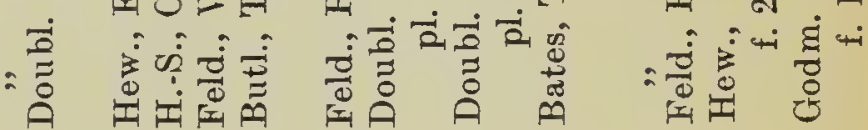

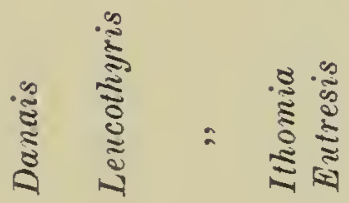

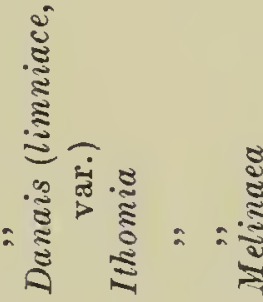

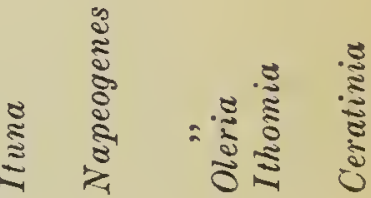

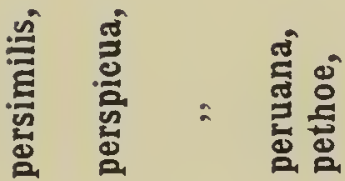

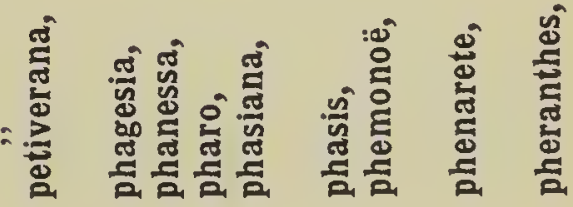

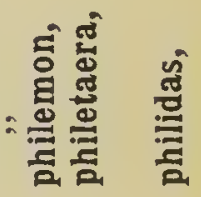

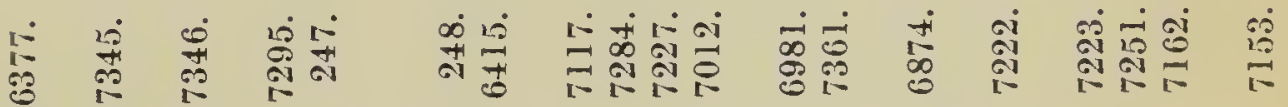




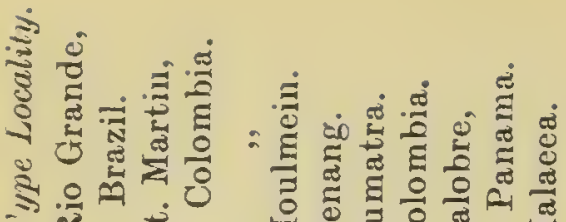

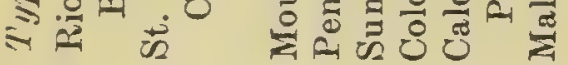
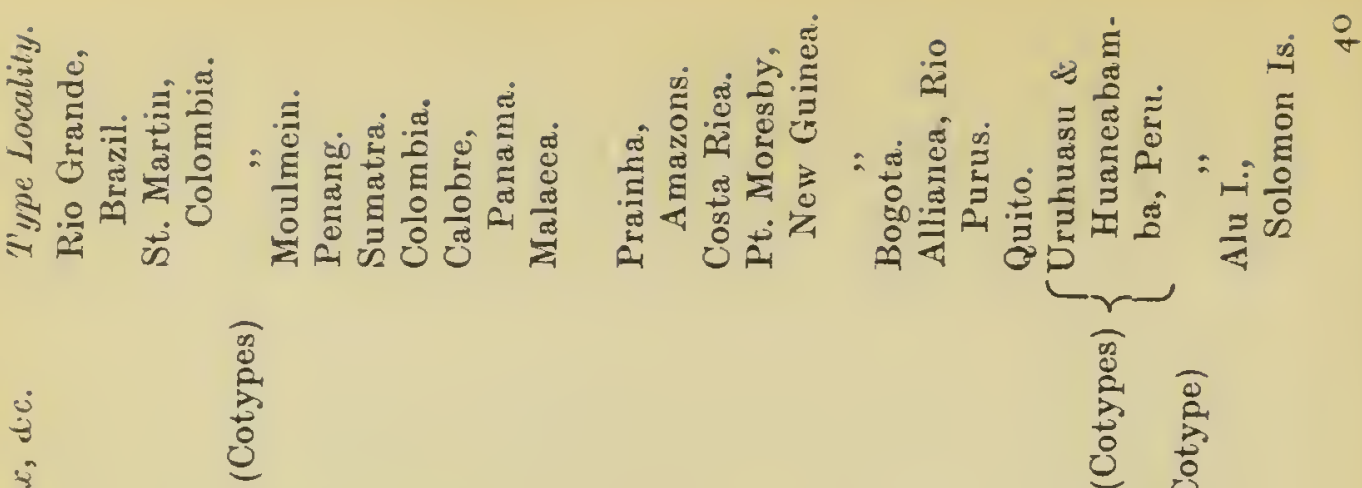

ot ro rorootrootot ro ro otro otroro ro ro

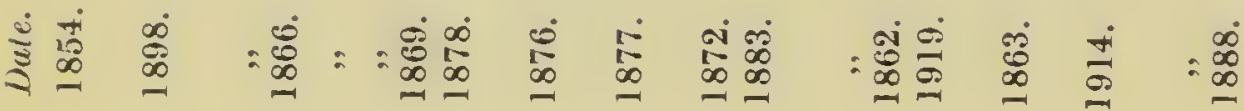

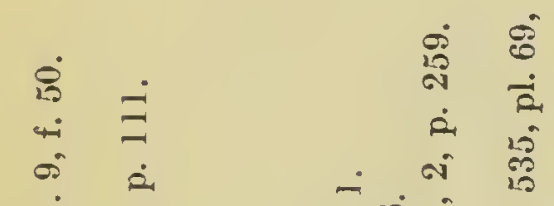

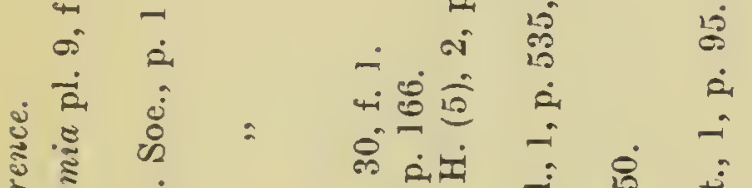

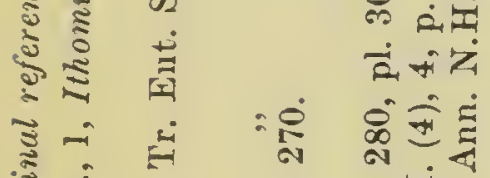

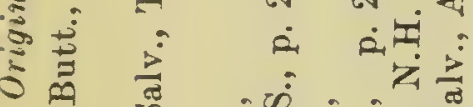

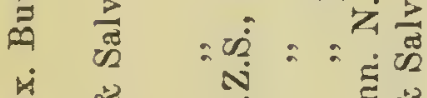

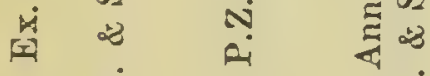

站
용

- $\dot{2}+\infty$

¿ ०

荘

$\stackrel{\sim}{\infty} \dot{0}$

is.

造 ì

ค.

का

की

竞离

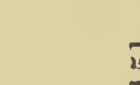

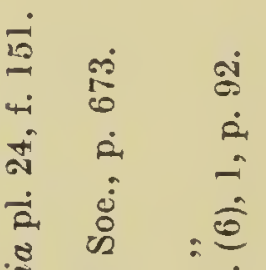

की

का

Eं

芶

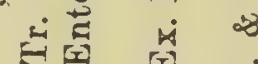

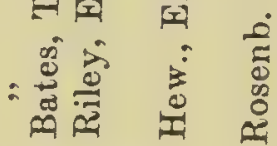

$\frac{20}{5}$

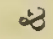

$=\frac{1}{0}$

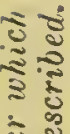

के

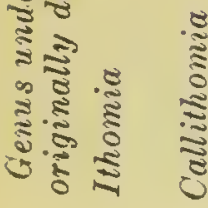

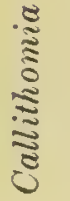

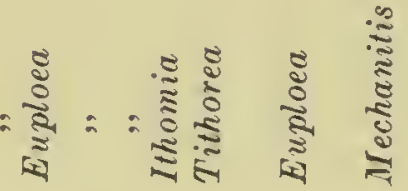

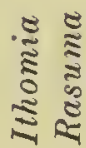

莡

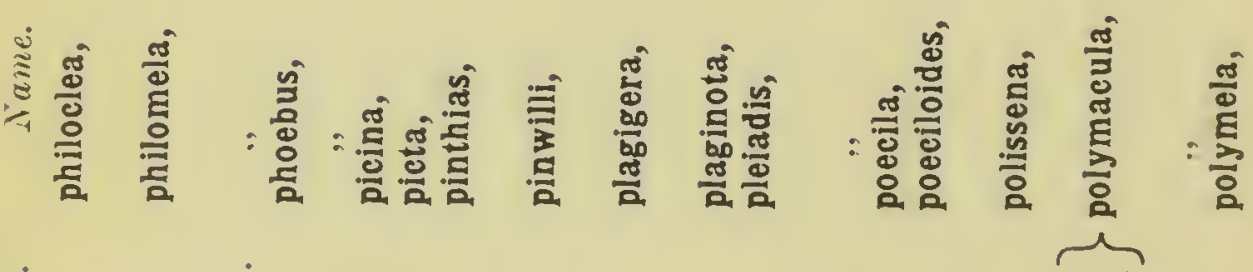

S-

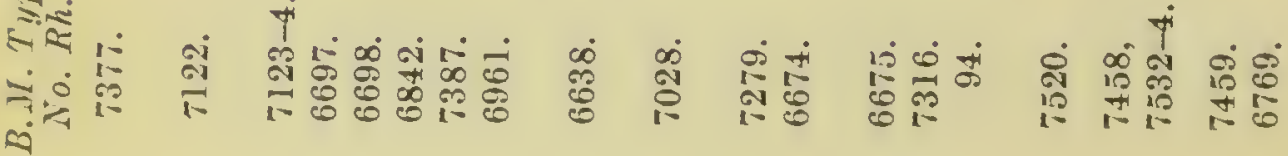




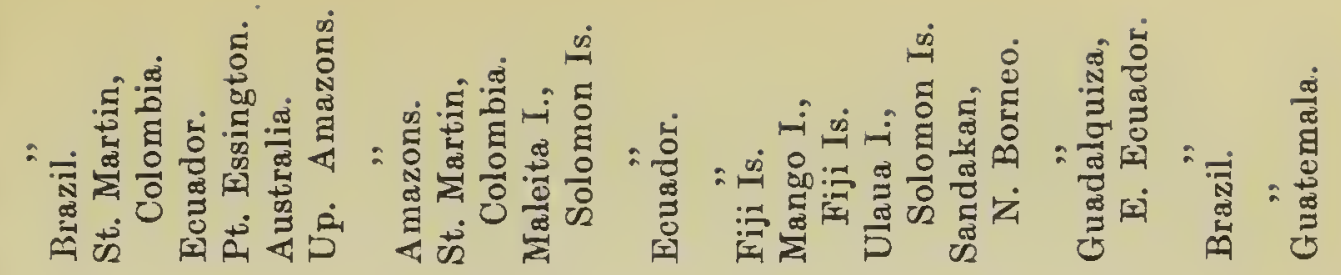

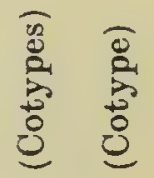

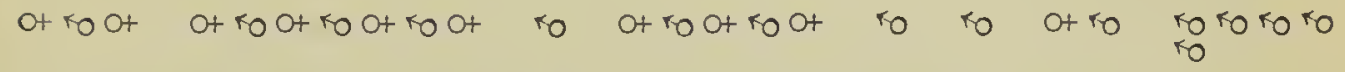

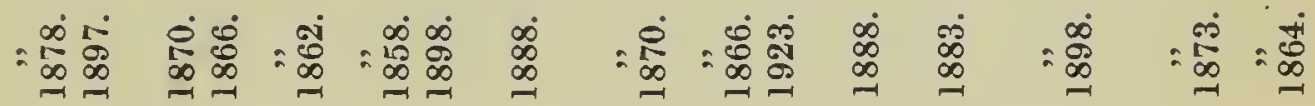

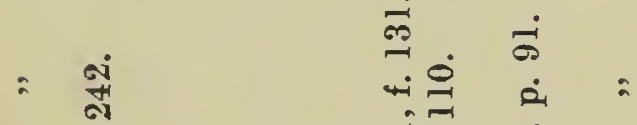

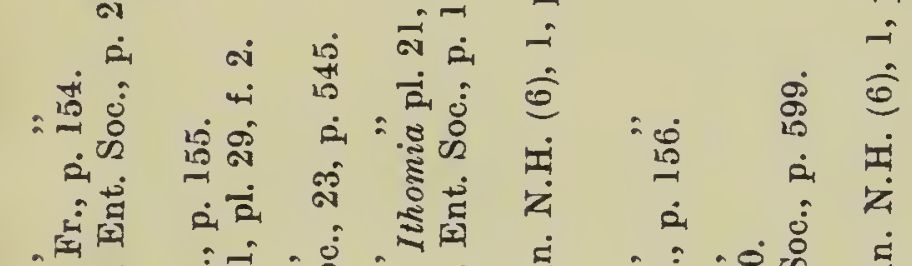

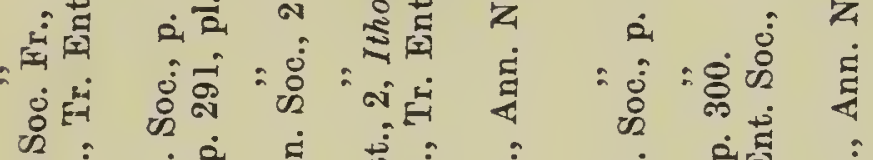

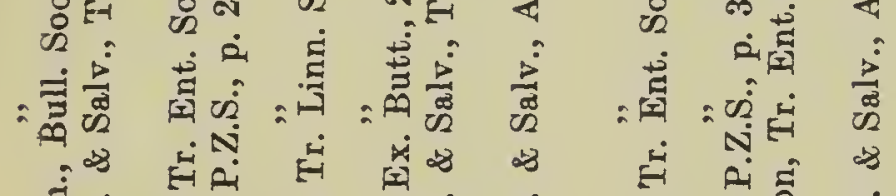

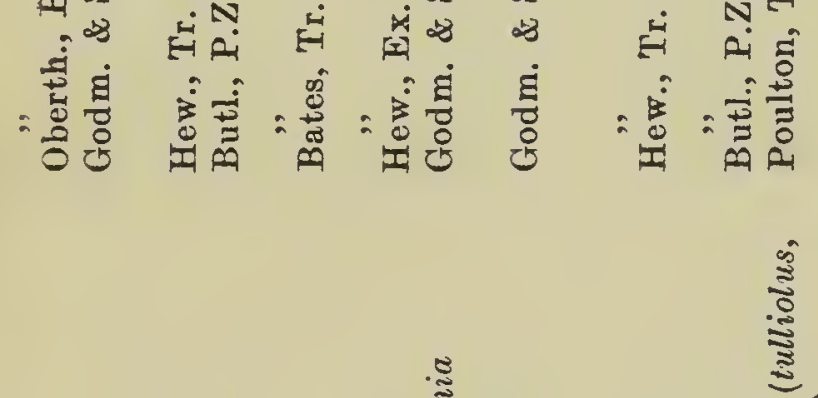

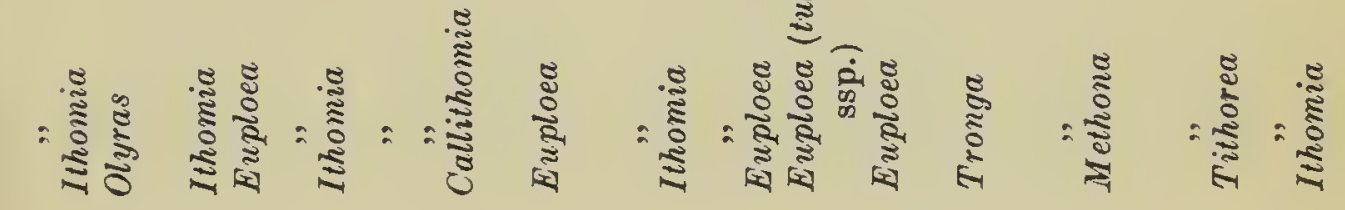

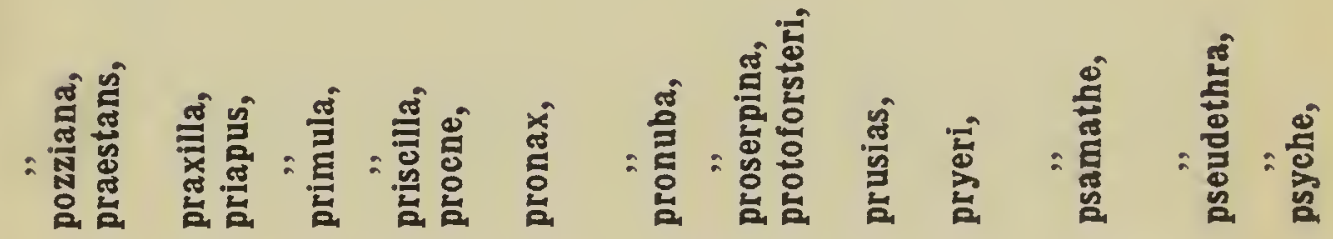

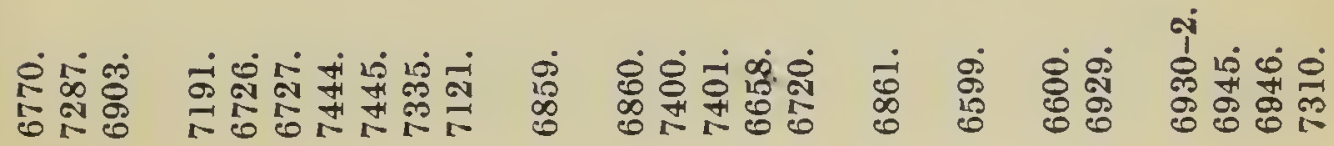


离

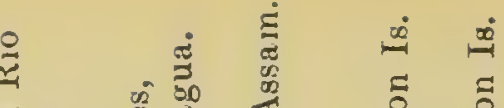

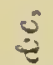

范

rootro rootrorootro otro atrootro ot rootro ot ot

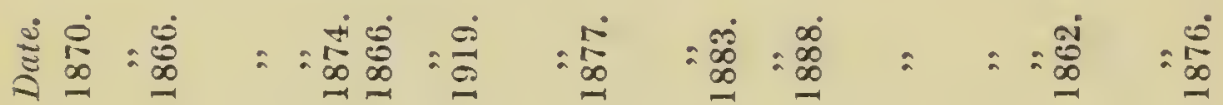

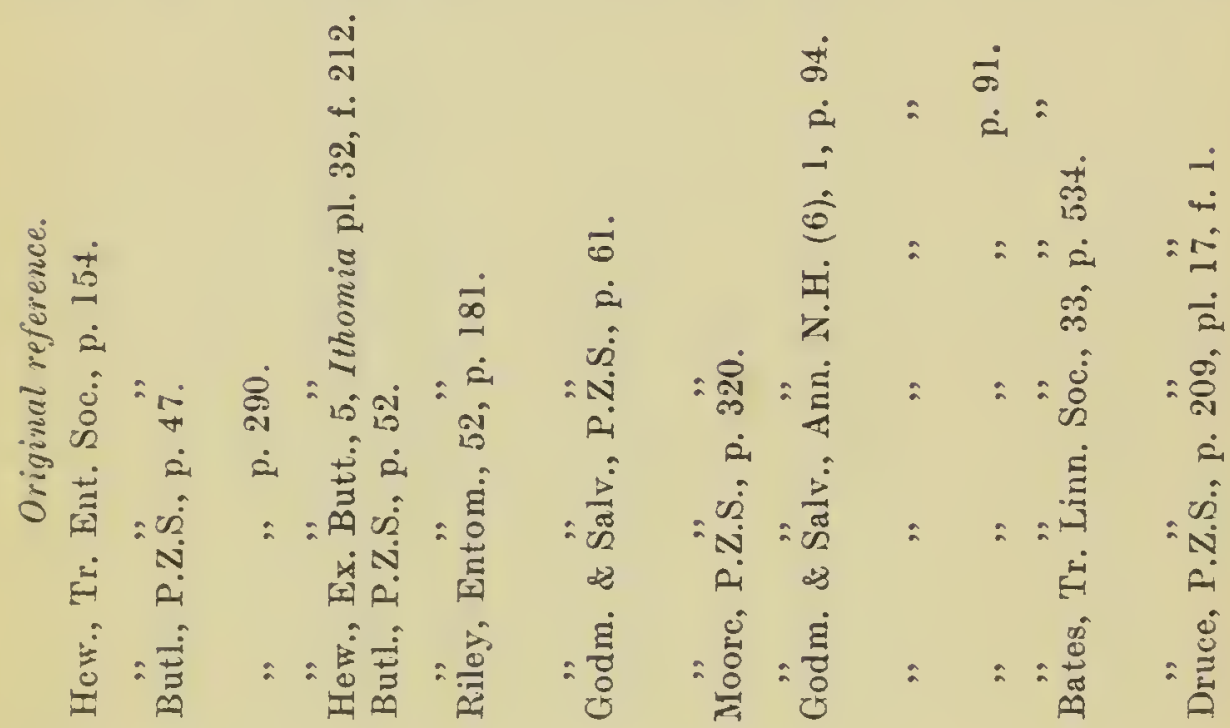

งิน

3.

골

¿

离. 莣

iั

$\stackrel{\widetilde{\Xi}}{\tilde{\Xi}}=\stackrel{3}{\tilde{\Xi}}$

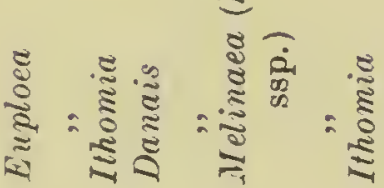

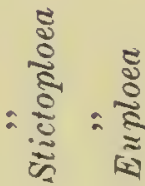

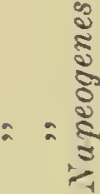

हैं

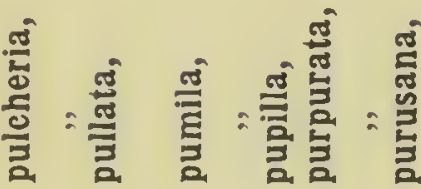

$=\frac{0}{\frac{0}{2}}$

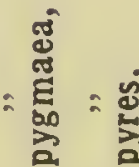

采

$\frac{0}{2}$

$\stackrel{\rightarrow}{\rightarrow \infty}$

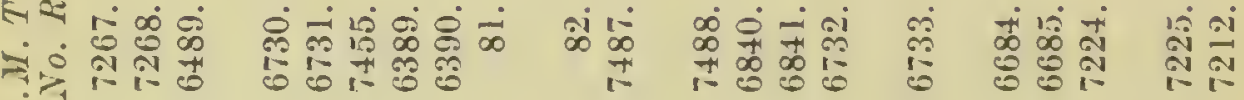
$\infty$ 


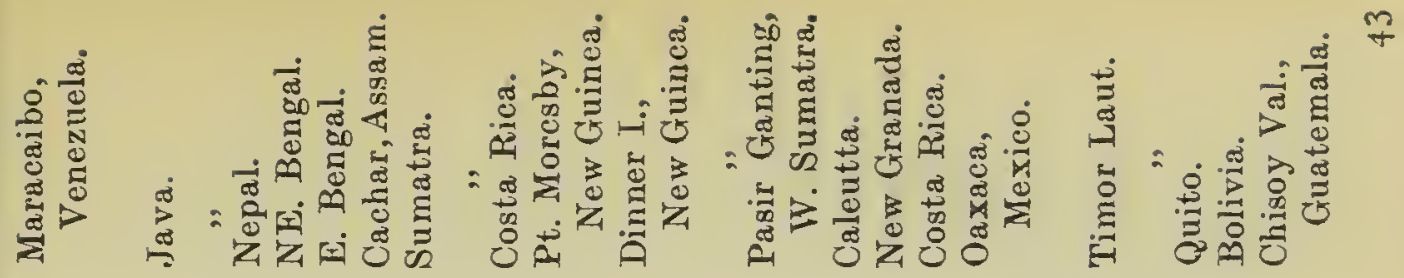

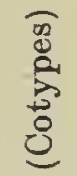

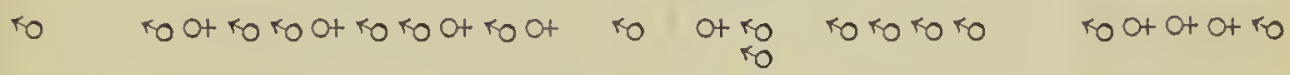

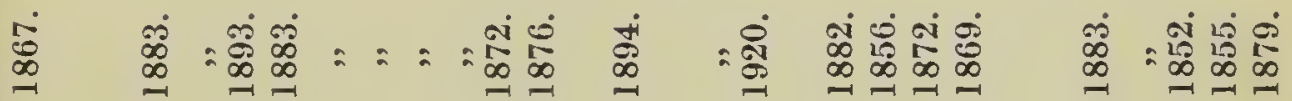

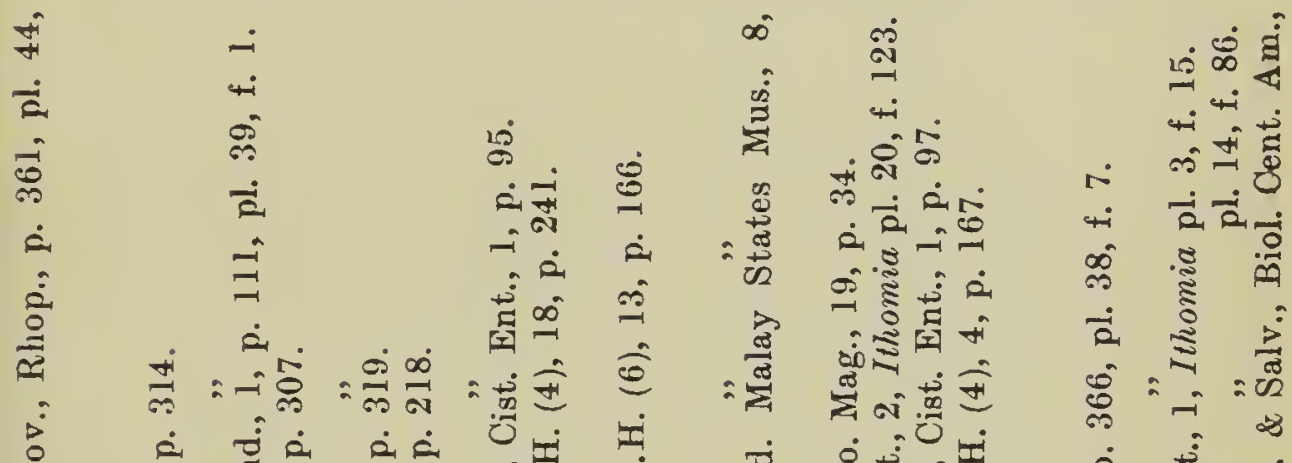

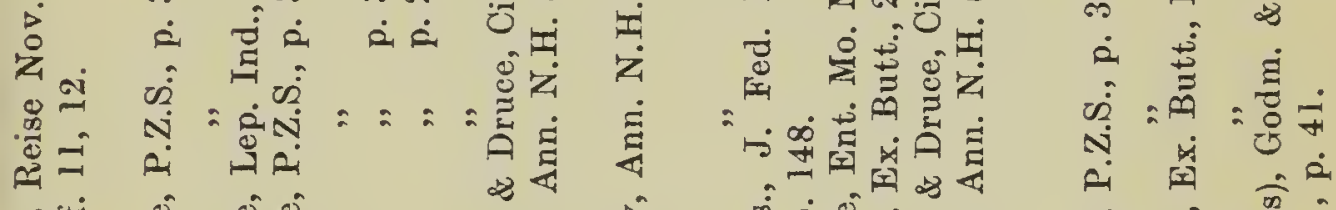

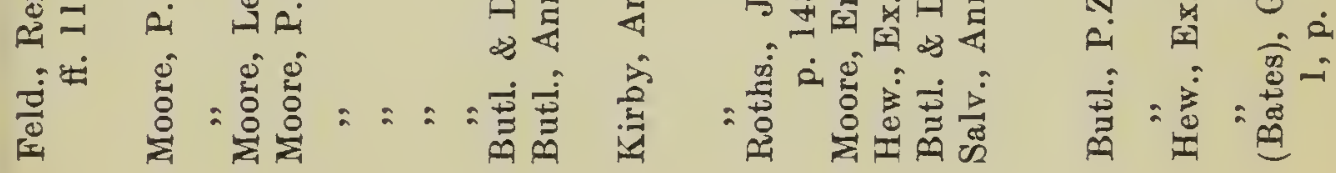

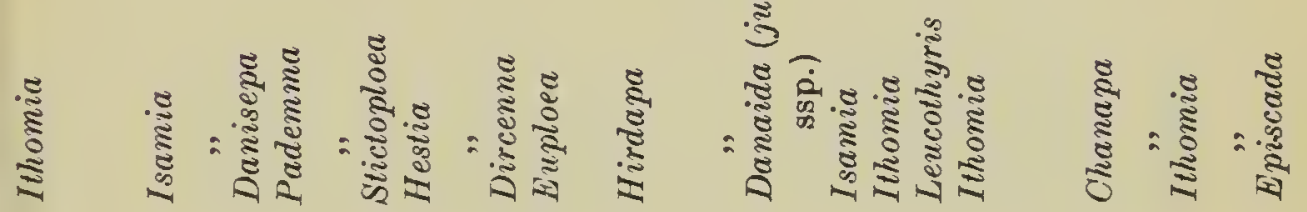

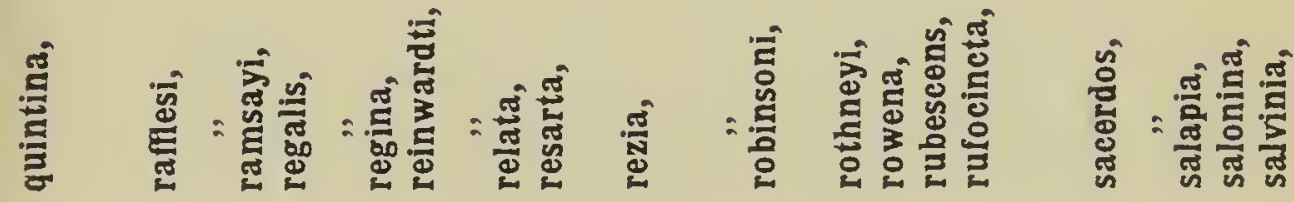

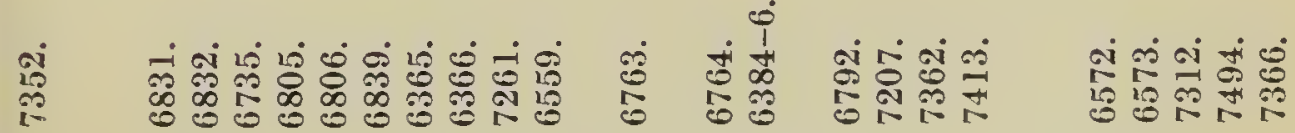




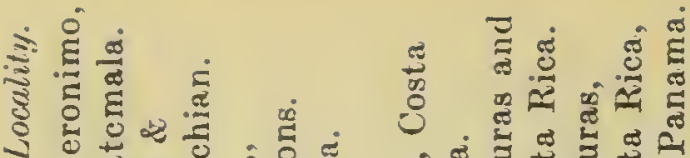

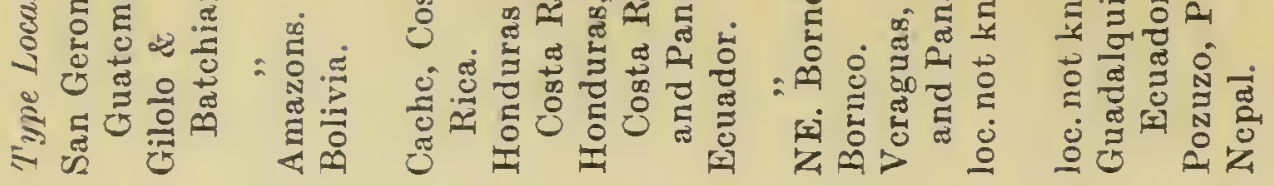

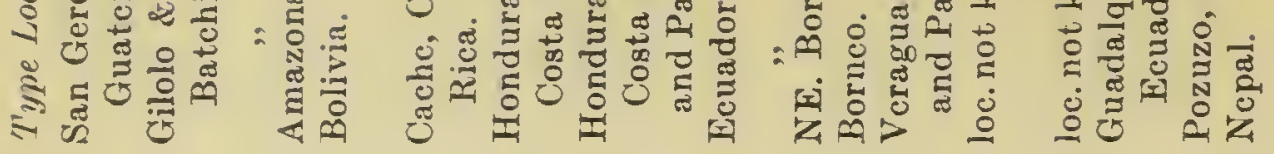

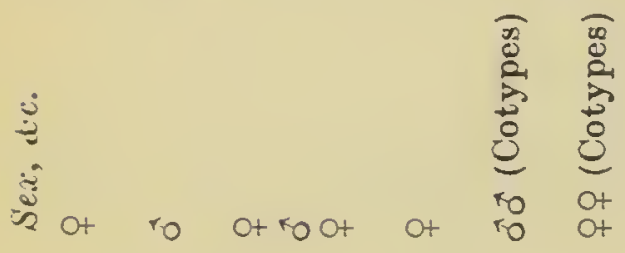

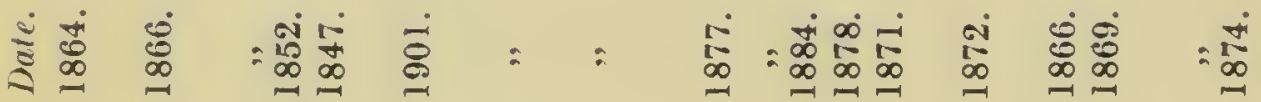

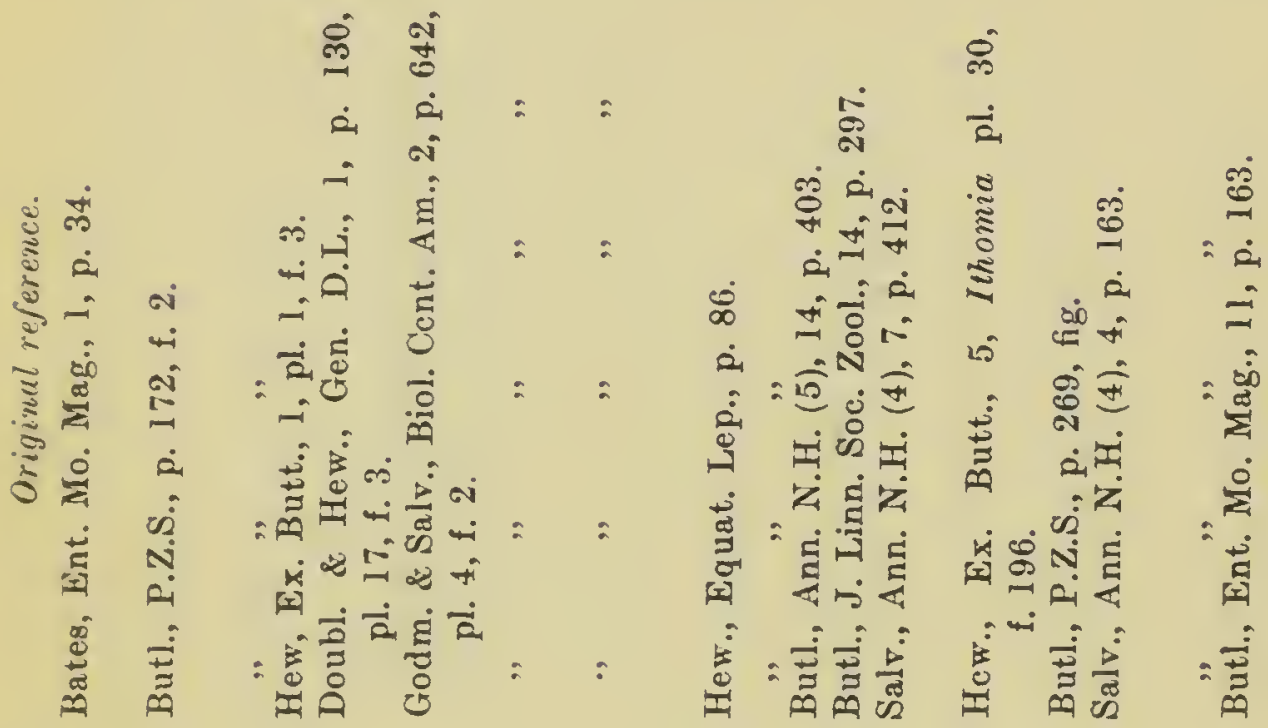

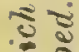

촐

มิ

츨

ว

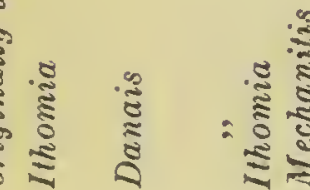

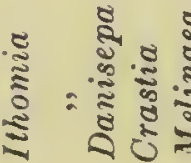

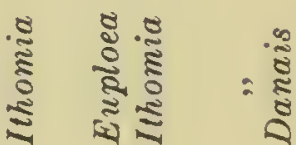

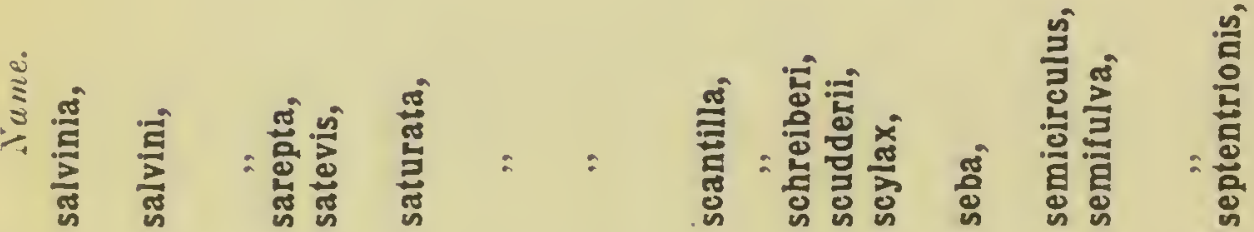

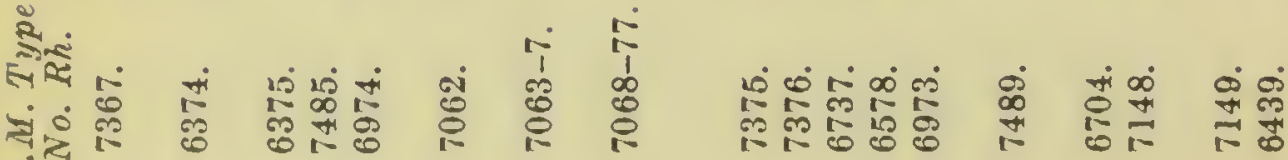
级 


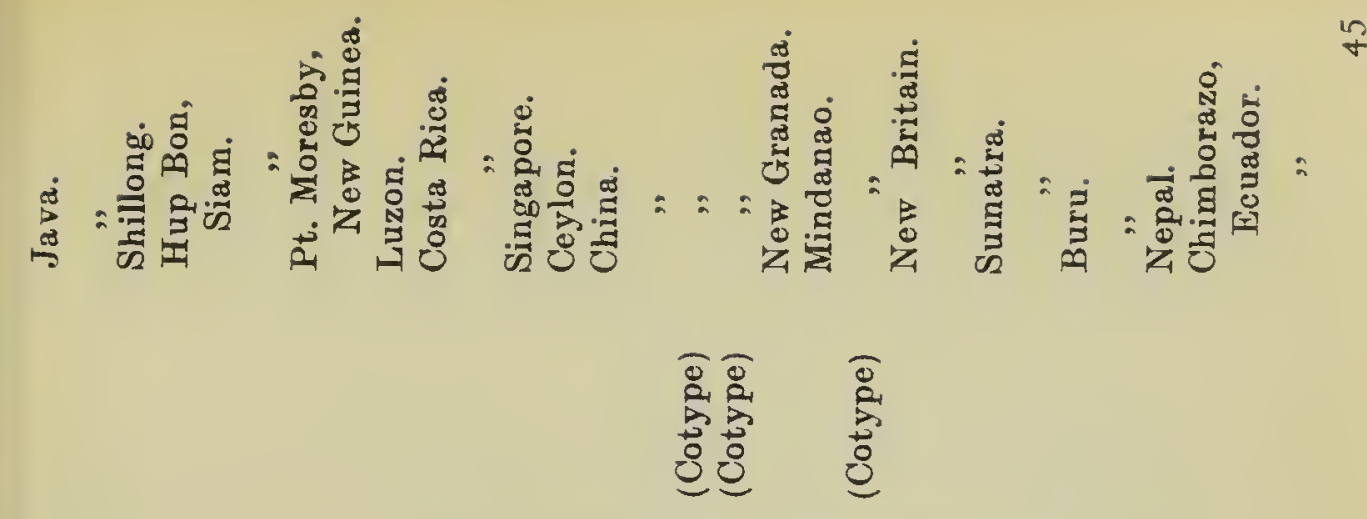

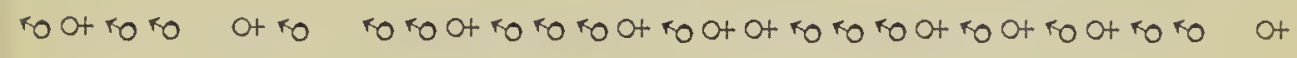

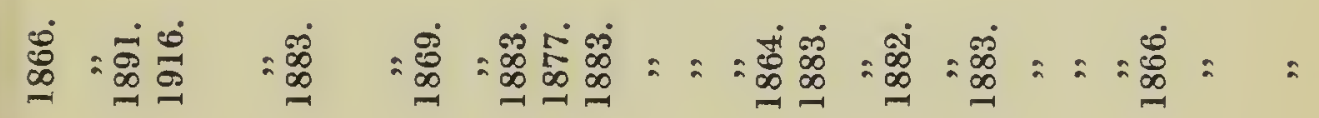

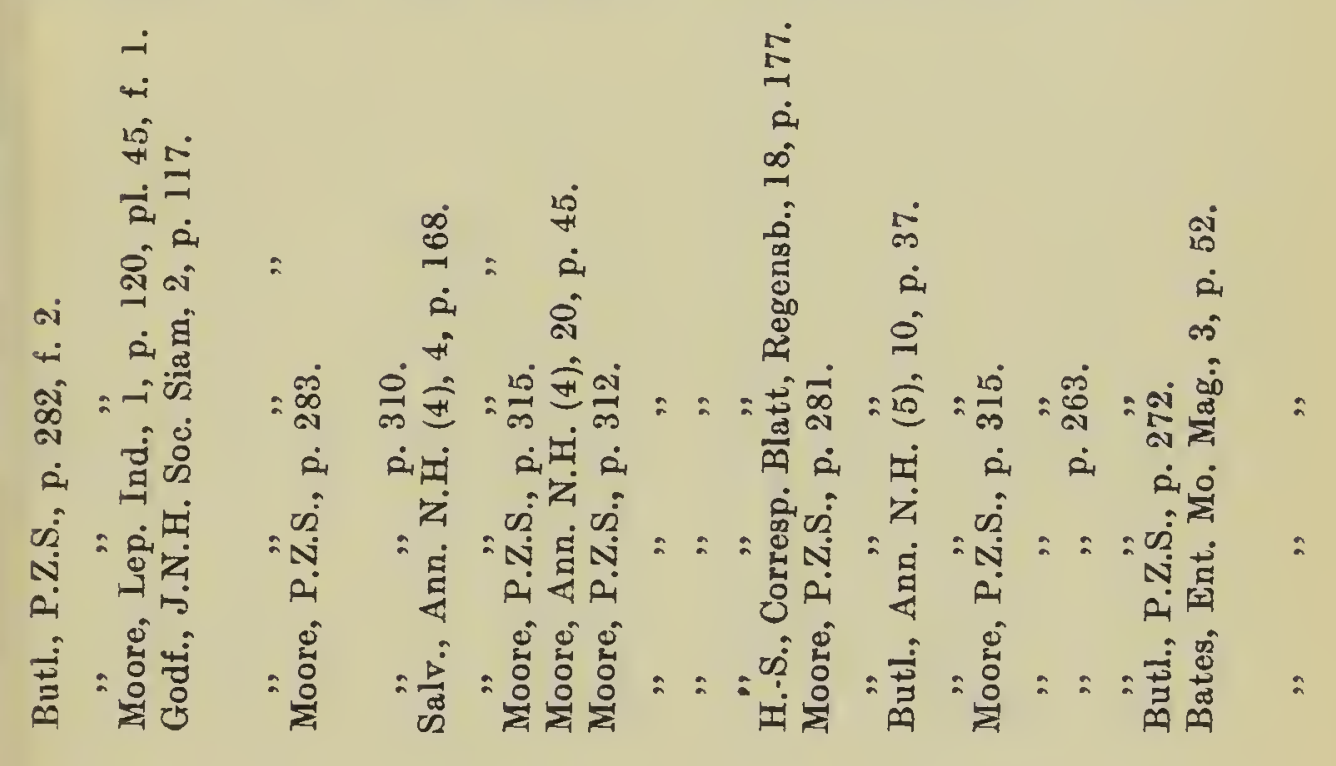

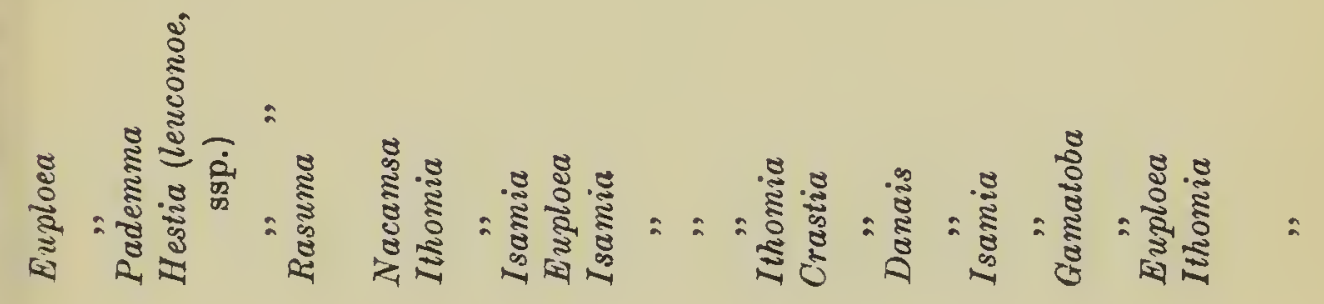

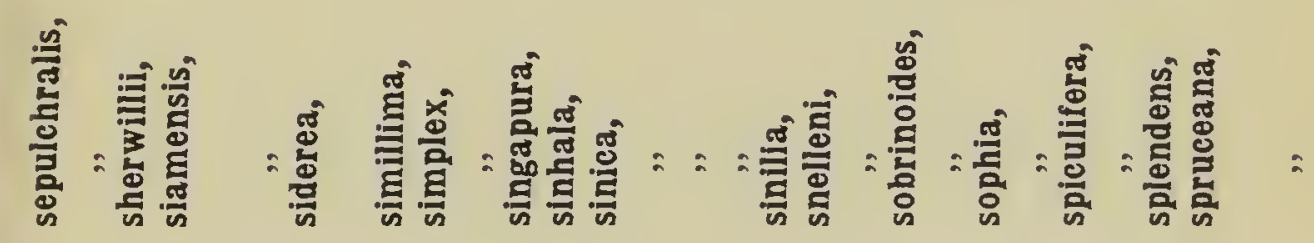

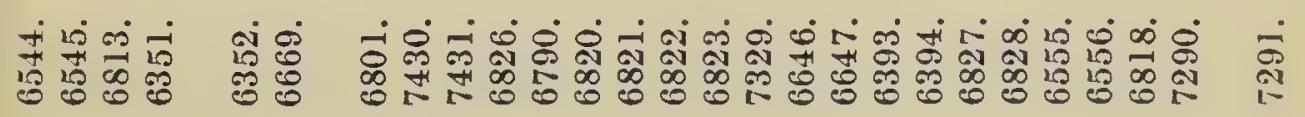




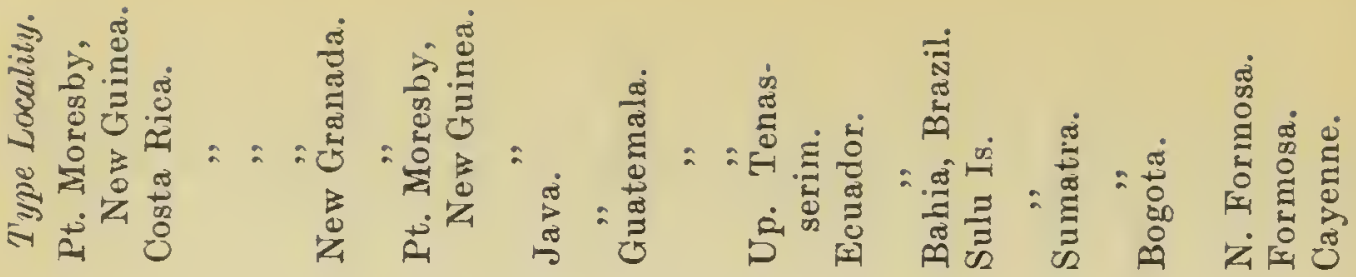

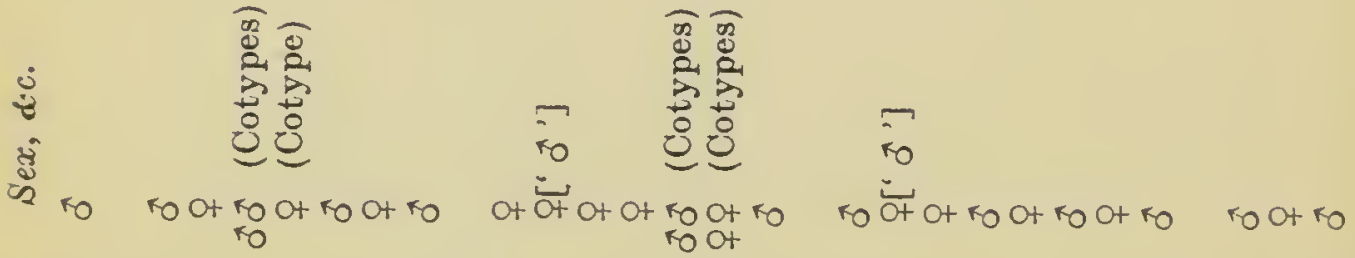

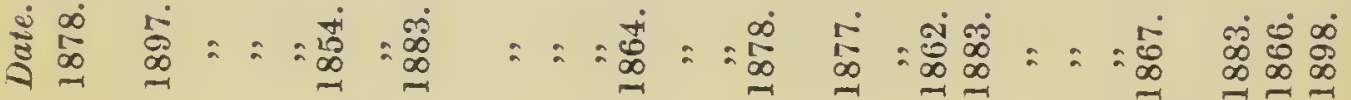

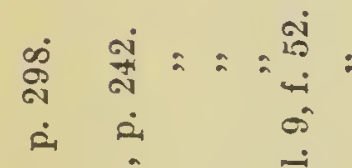

过

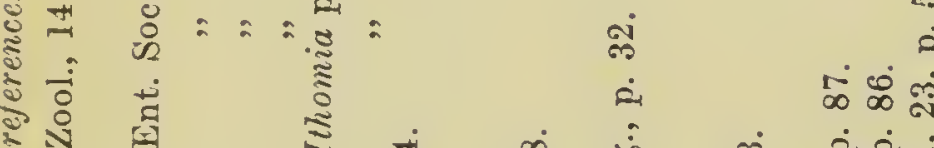

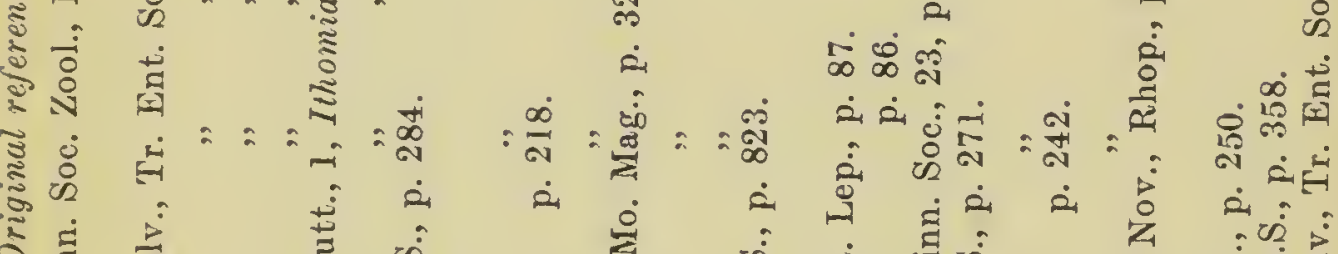

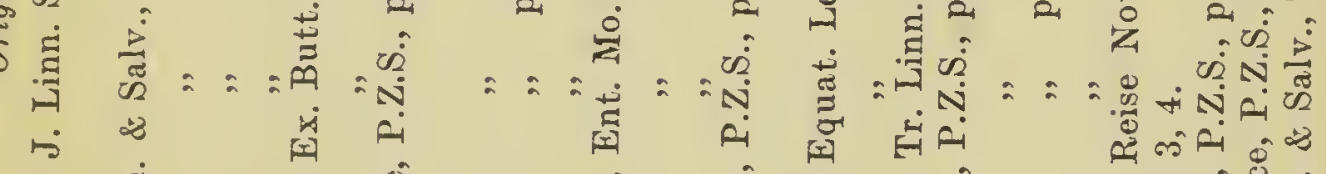

莣

ชั่

$\stackrel{2}{2}$

के

$\stackrel{2}{\pi}$

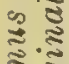

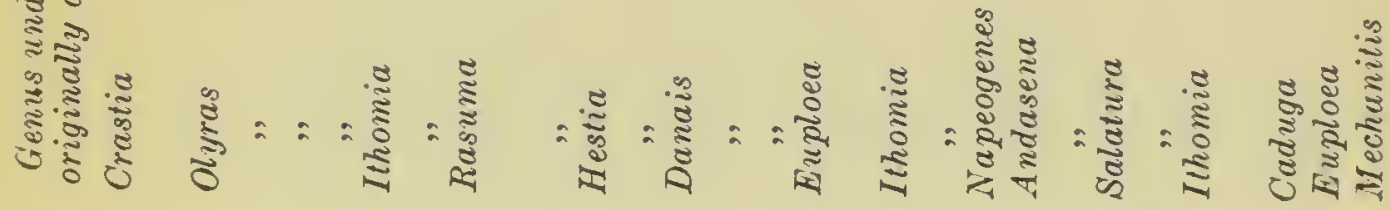

倍

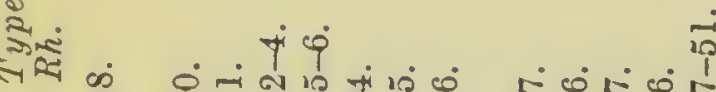

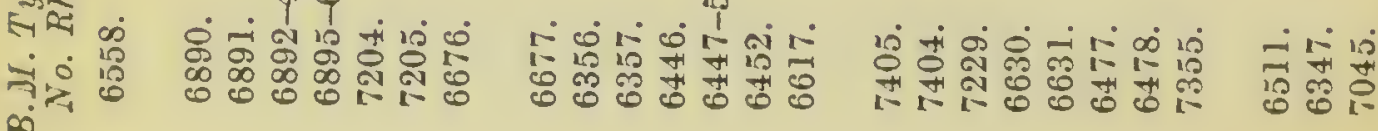




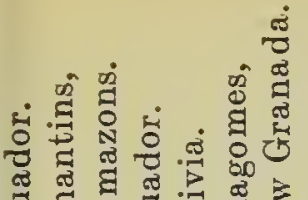
ठ (ํ)

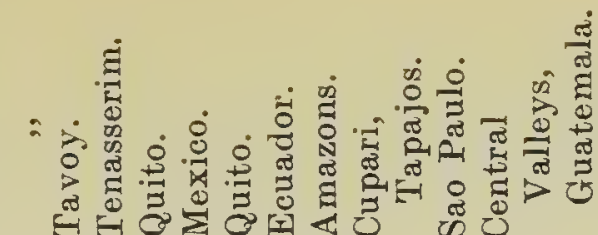

root rootro of ro root rot of of ro of

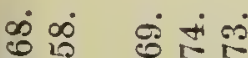

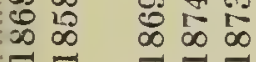

(5)

in

ติำ

$\dot{2} \overrightarrow{0}$

8

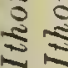

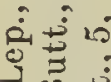

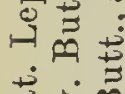

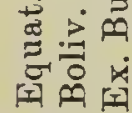

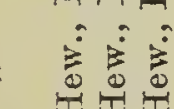

证要

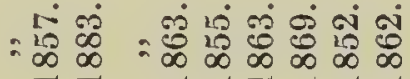

$-$

स- $\quad$ is

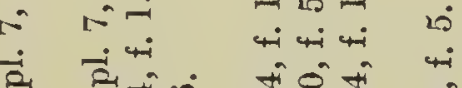

सं जी जी N

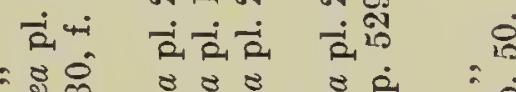

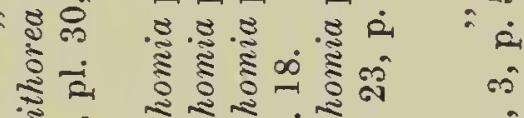

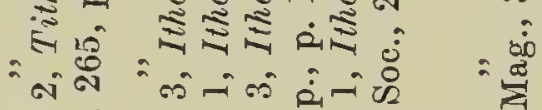

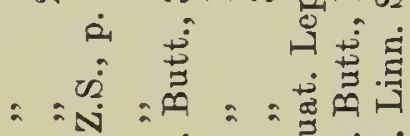

Li

昰点点

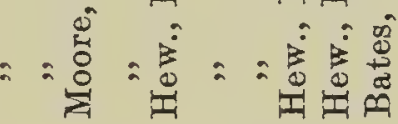

$\therefore \infty_{\infty}^{\infty}$

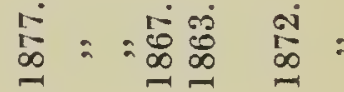

root roto root
root

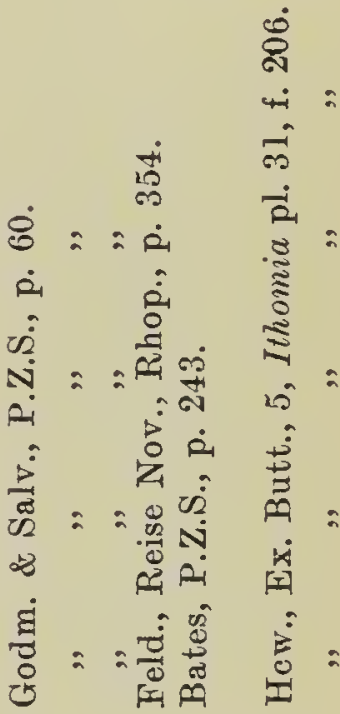

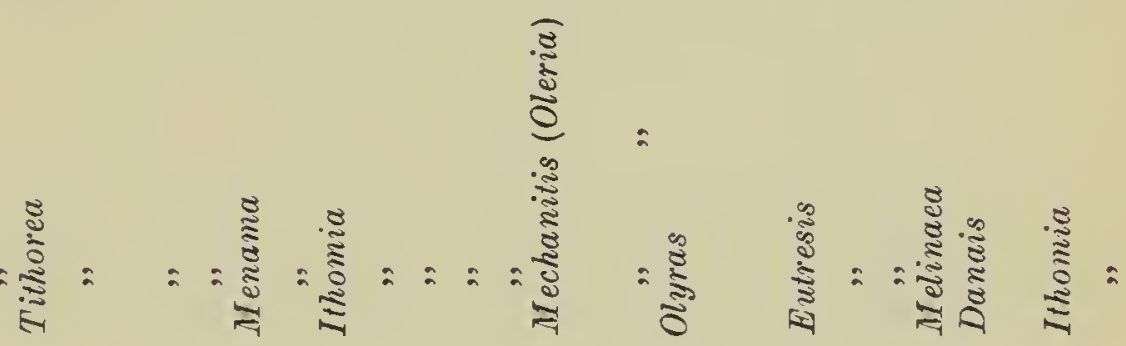

ind

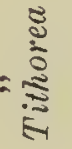

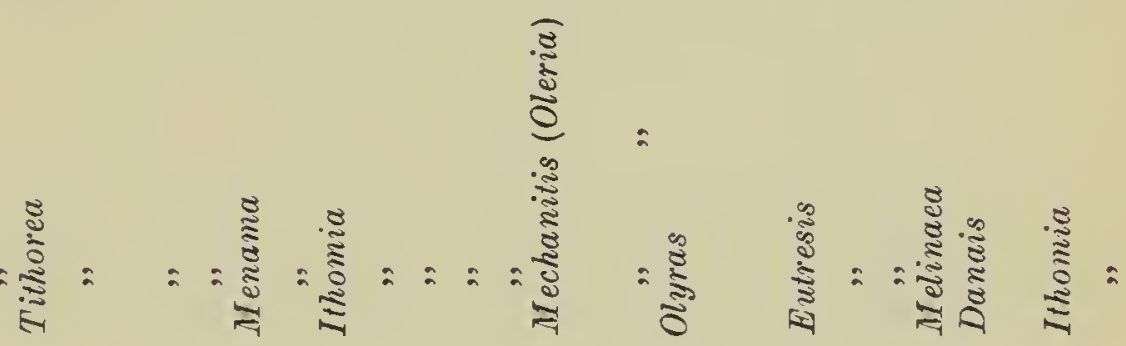

$=\frac{0}{\infty}$

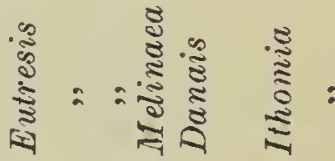

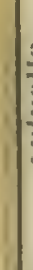

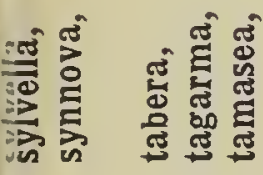




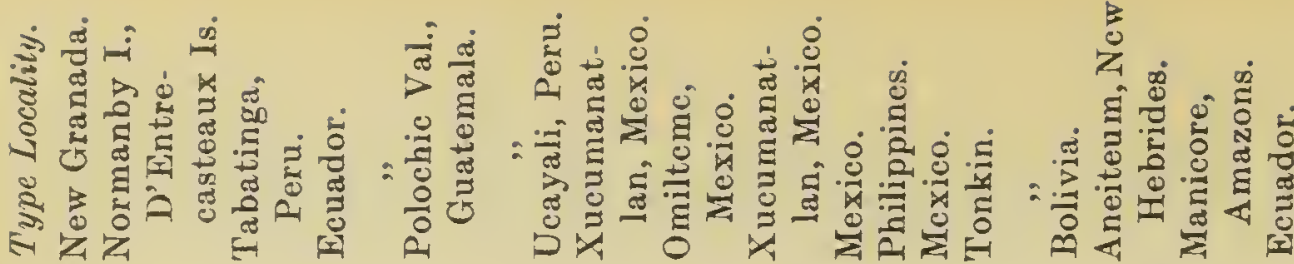

告告

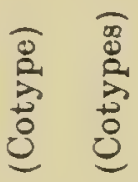

8ิ

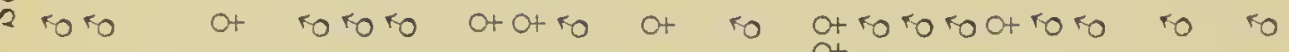

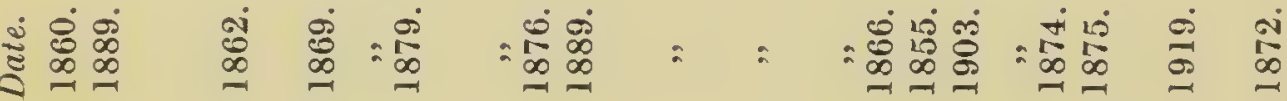

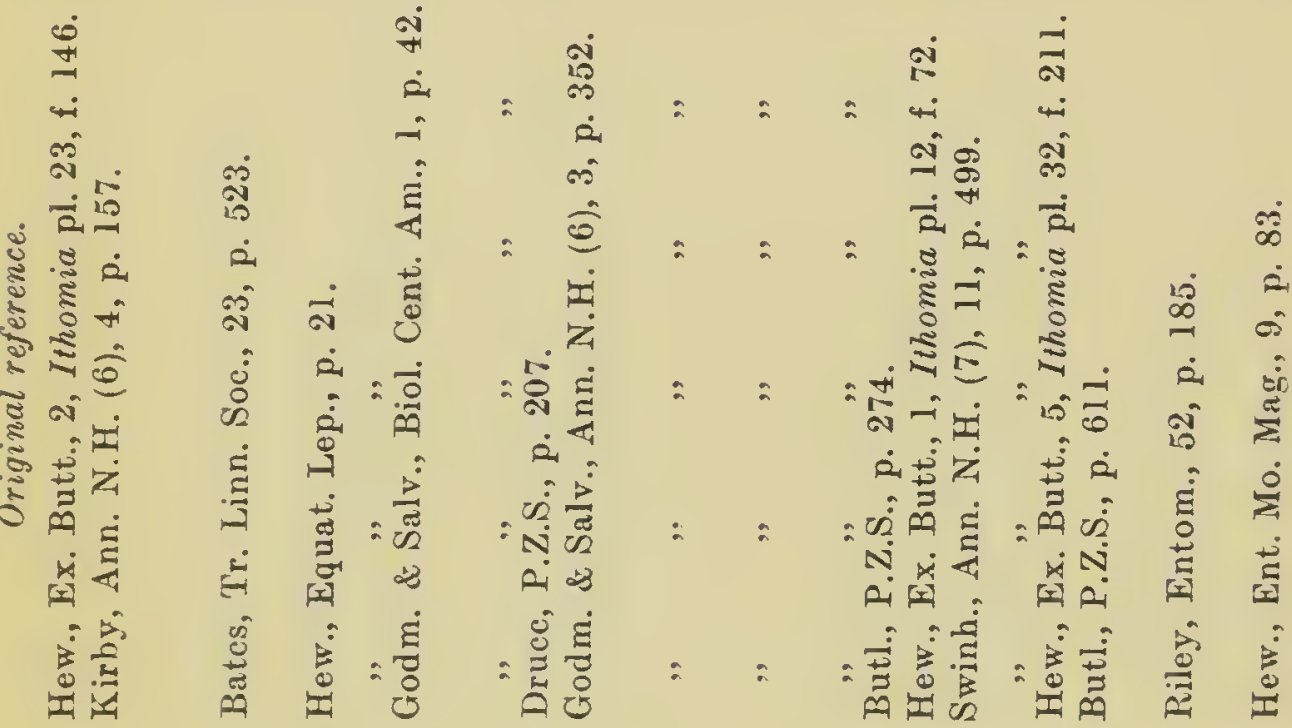

3

इำ

ระ

$\stackrel{2}{\pi}$

3 .

$\stackrel{8}{(3)}$

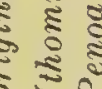

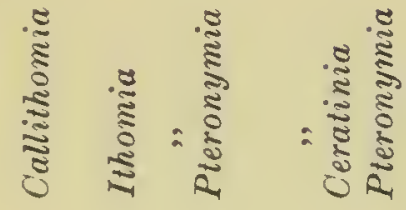

ఏँ

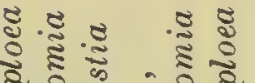

कूँ

बंग

空蒙

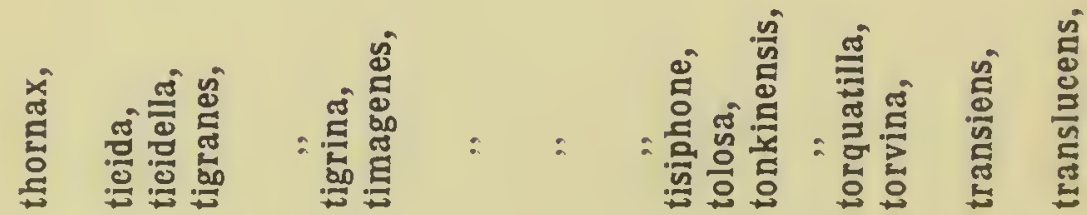

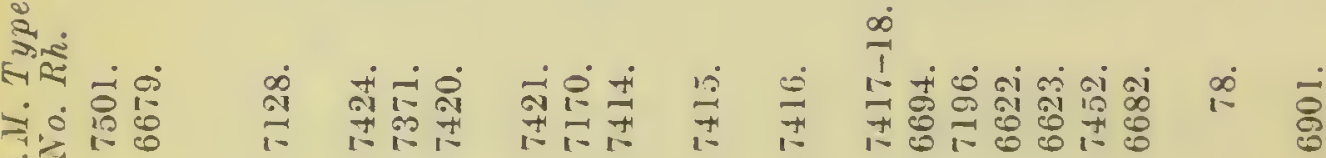




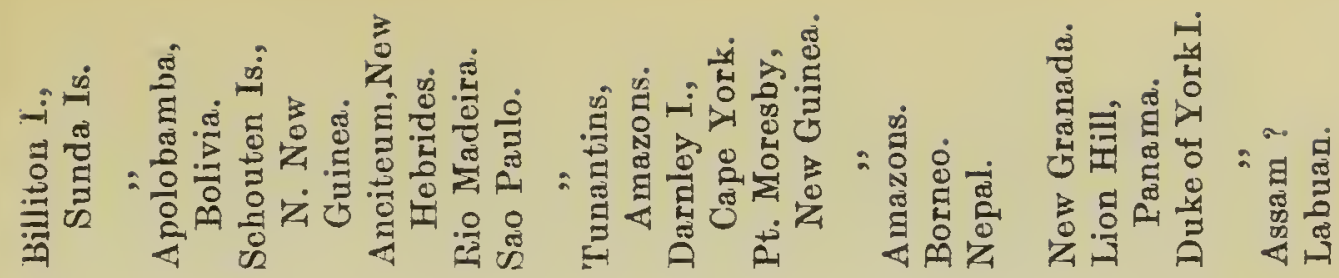

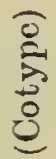

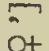

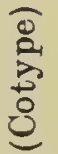

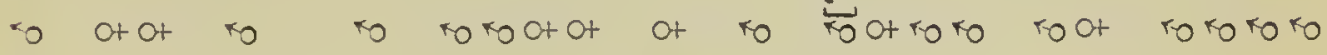

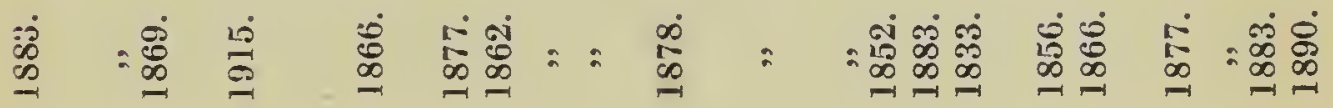

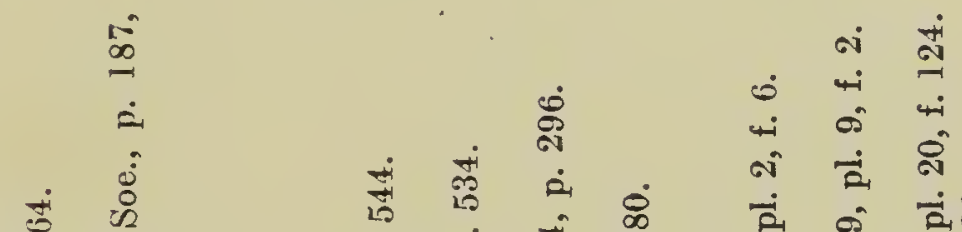

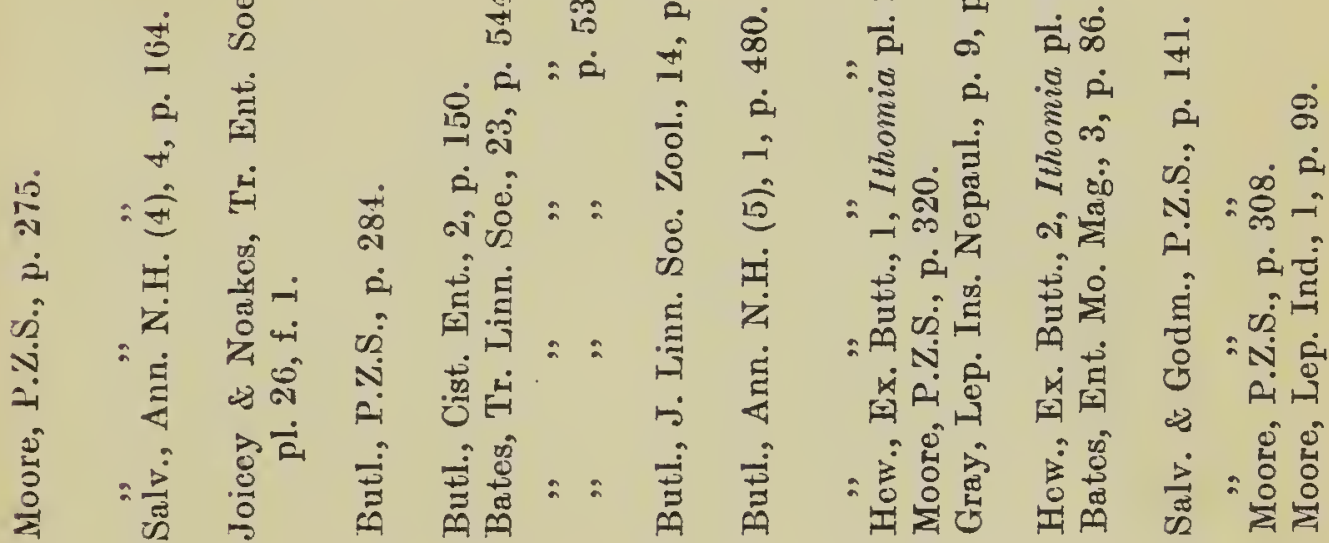

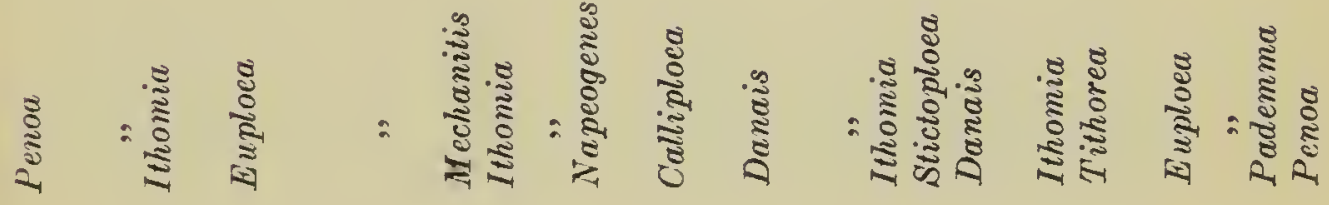

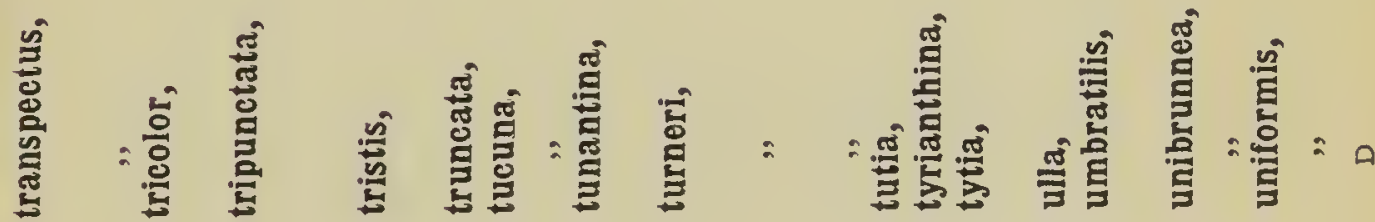

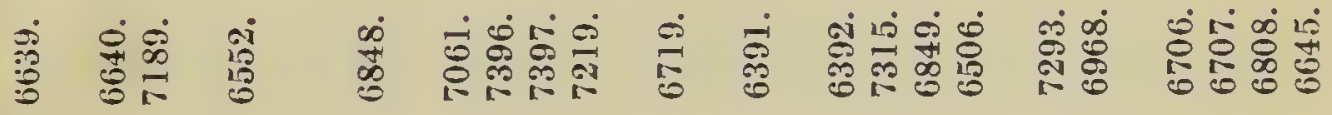




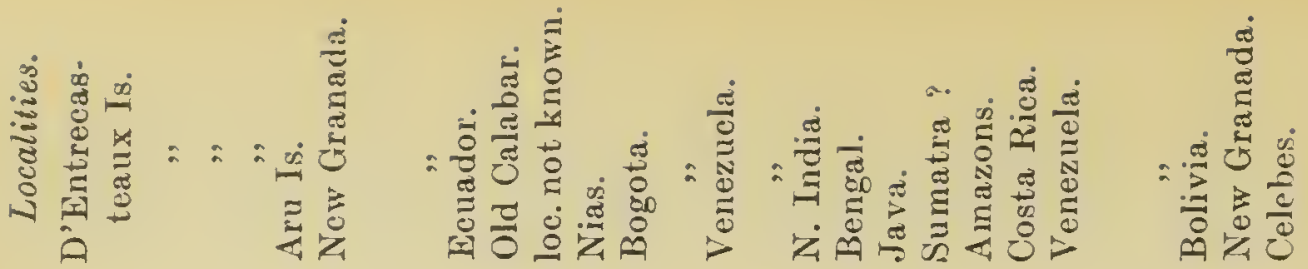

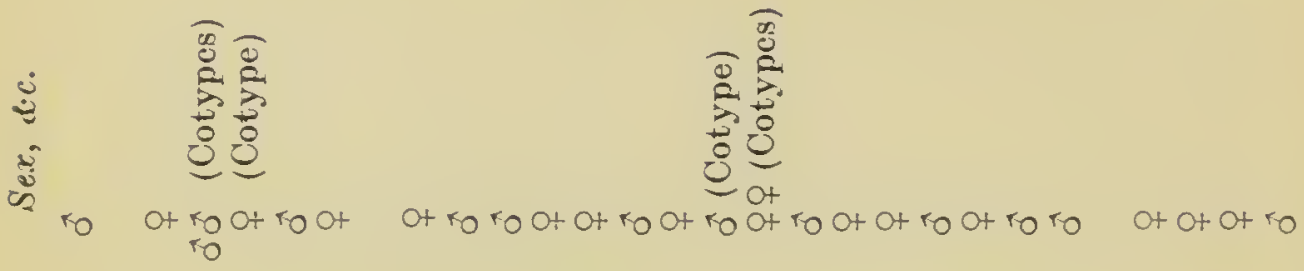

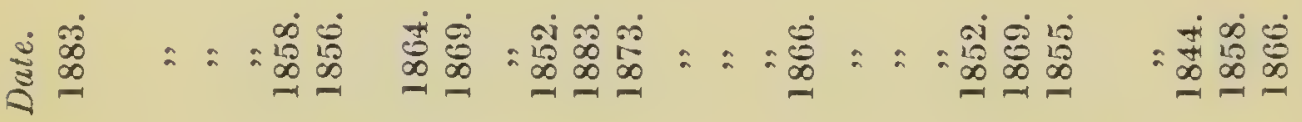

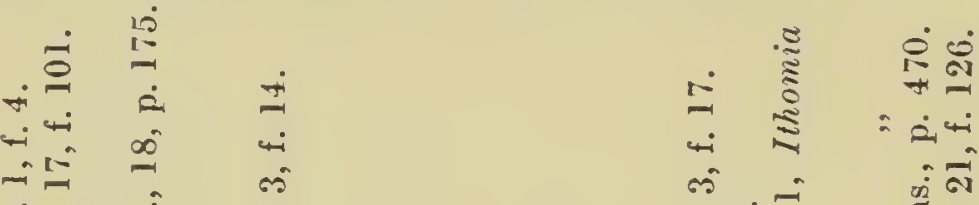

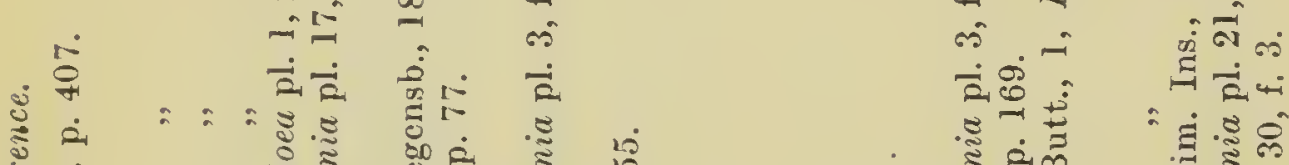

चँ

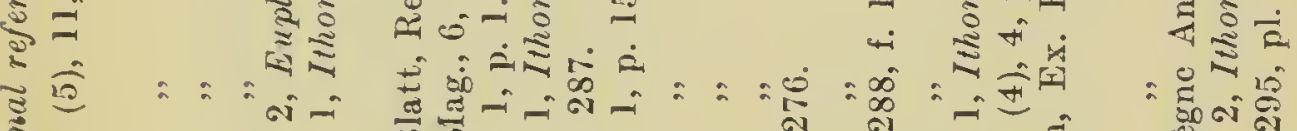

ज्ञ

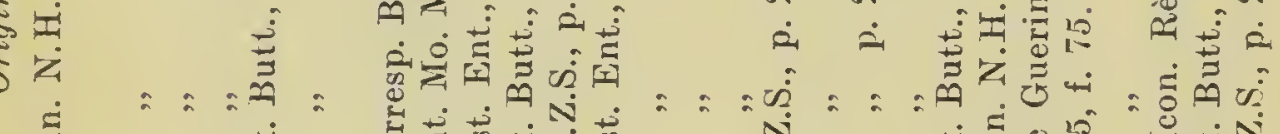

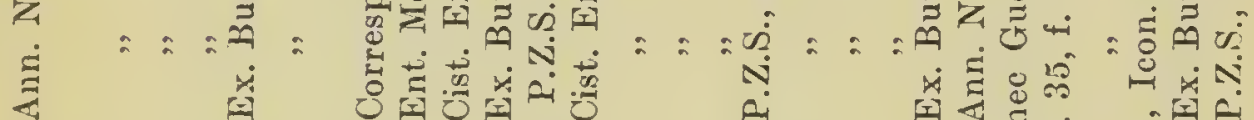

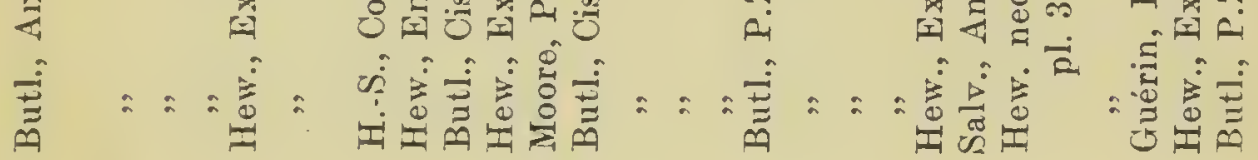

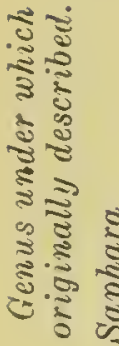

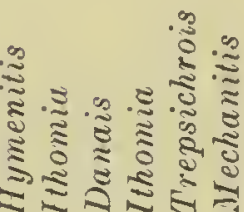

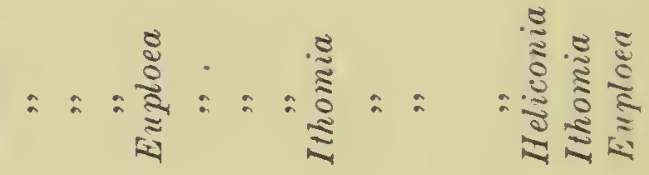

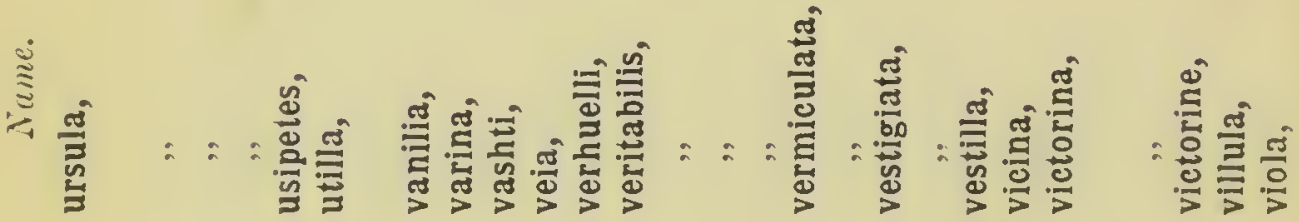

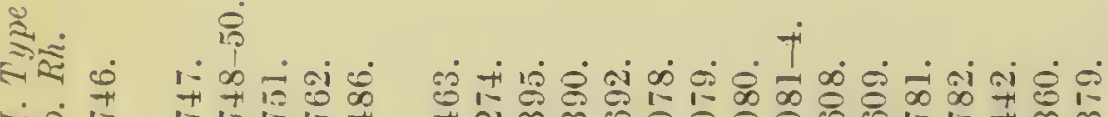

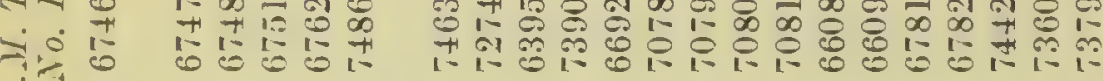




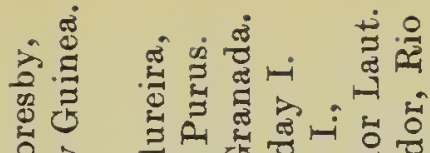

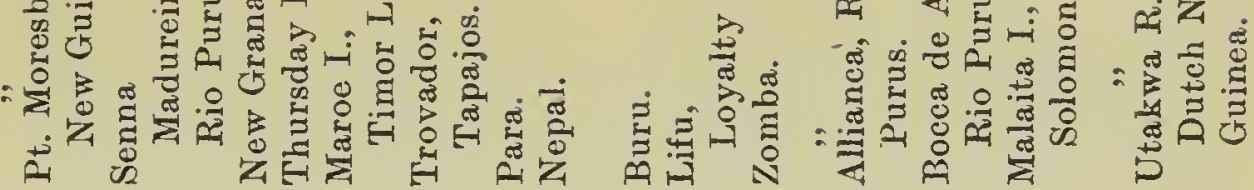
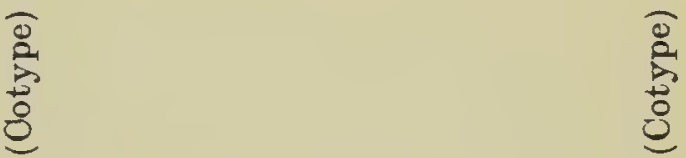

ot ot ot ot ro ro roro rot rot rot rot

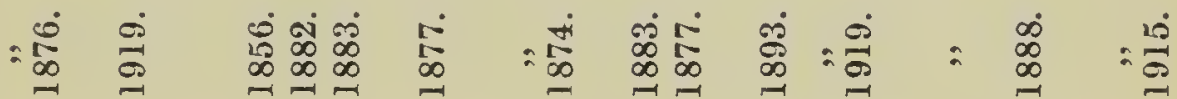

$\stackrel{\infty}{\rightarrow}$

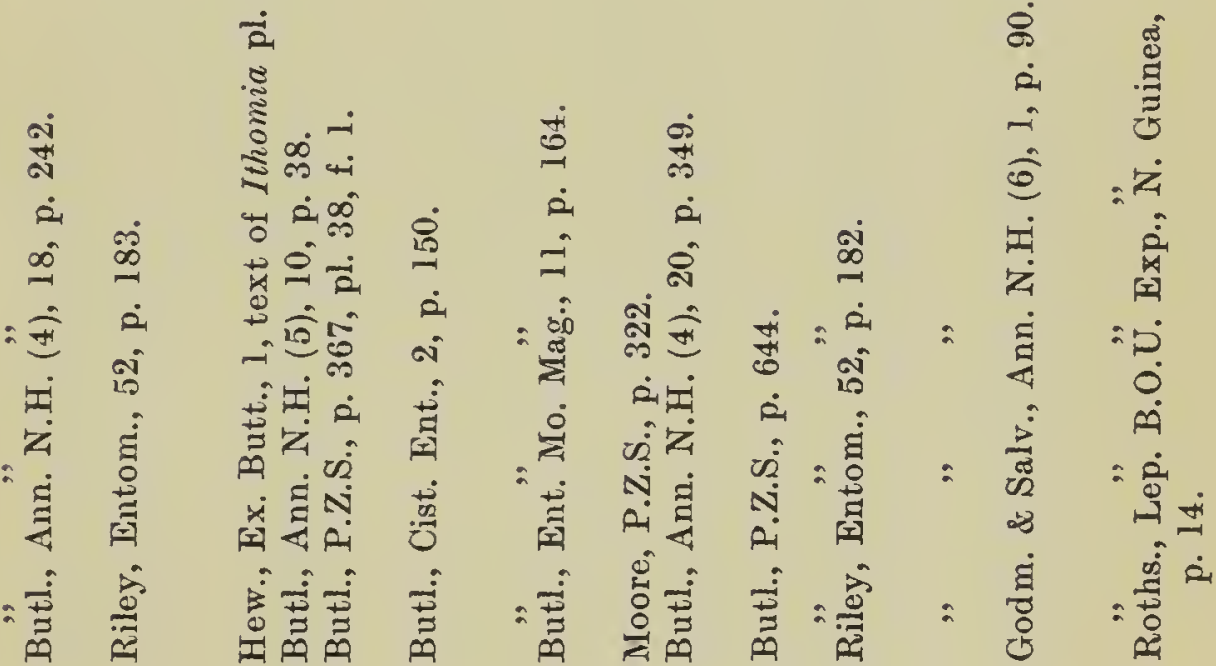

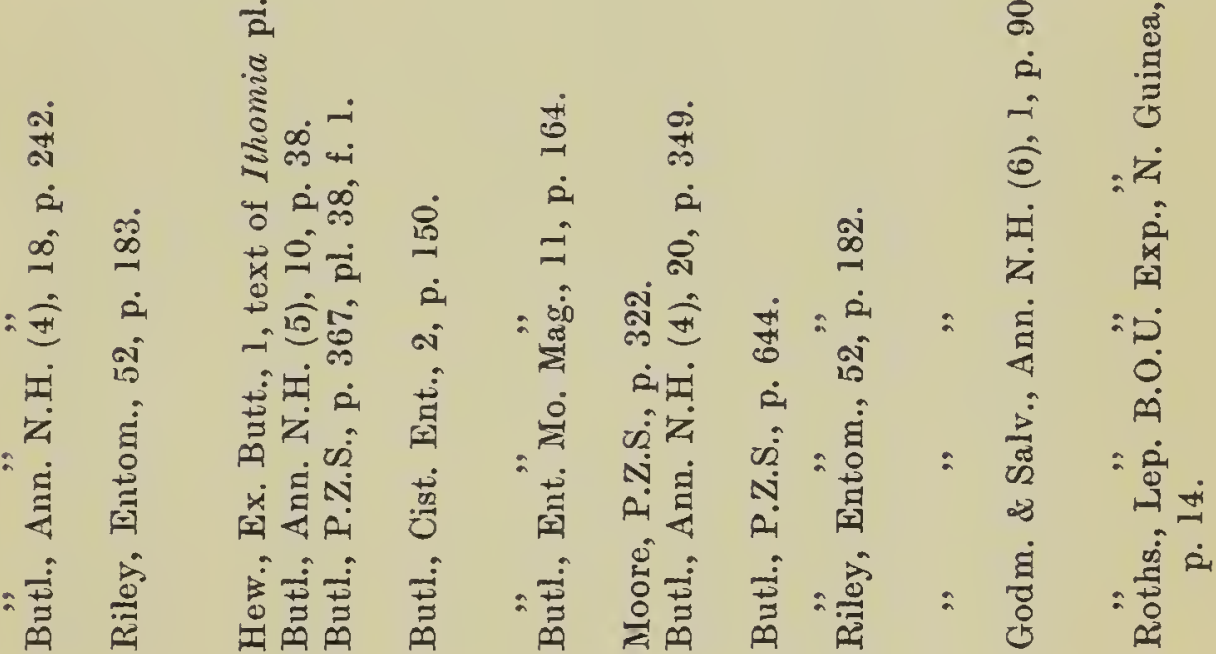

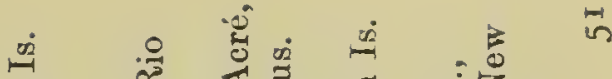

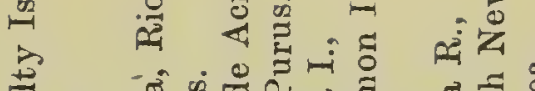

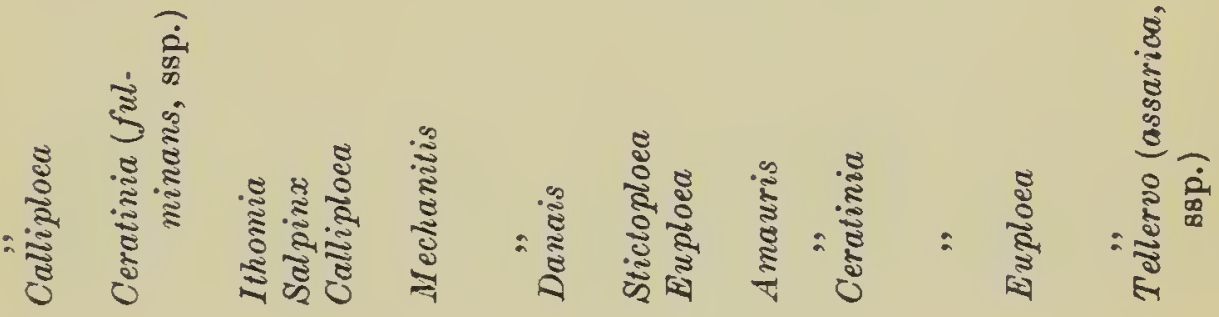

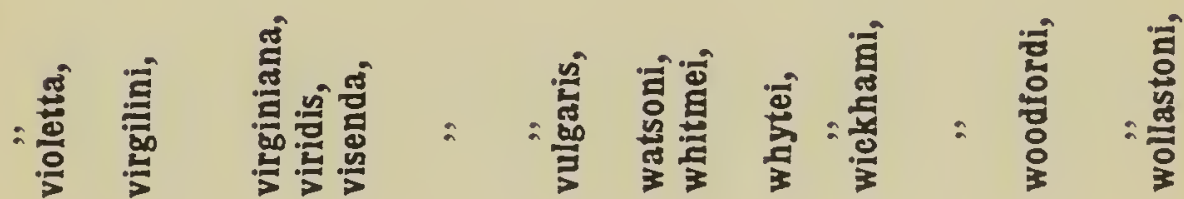

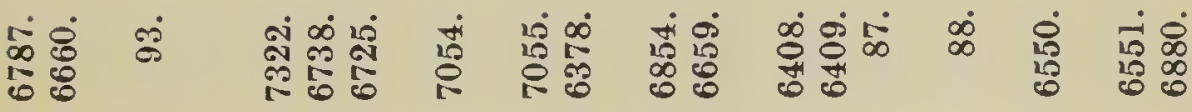




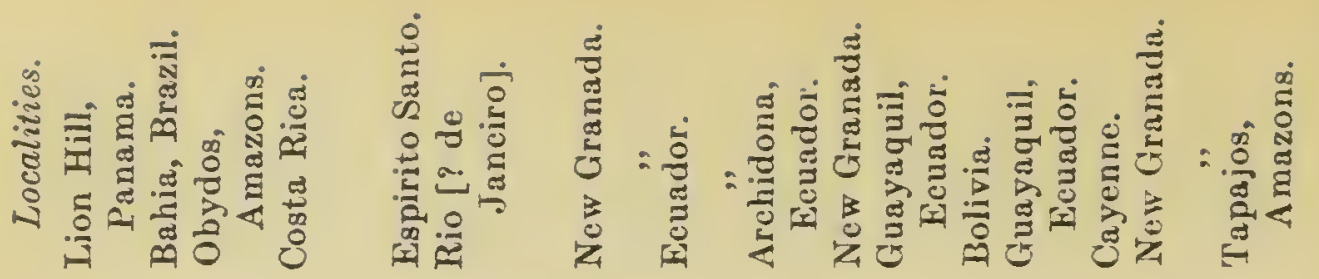

¿ُ

है

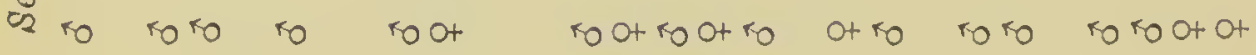

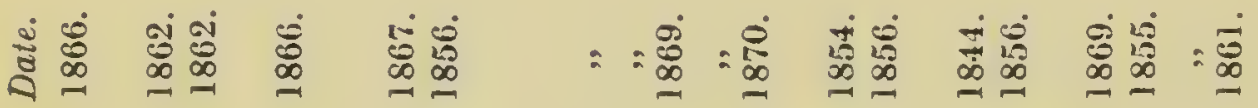

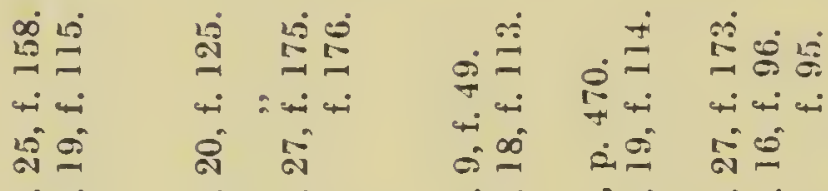

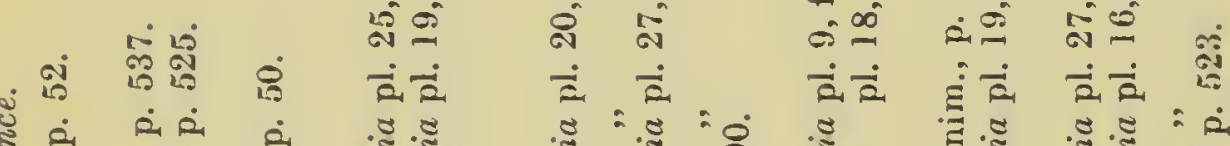

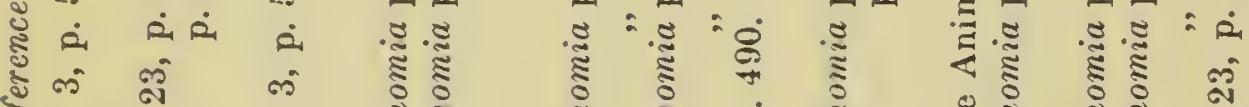

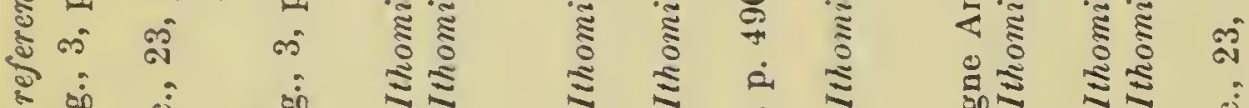

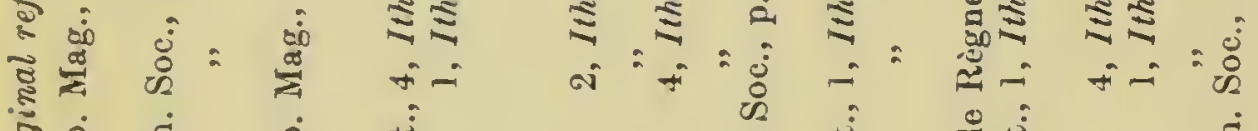

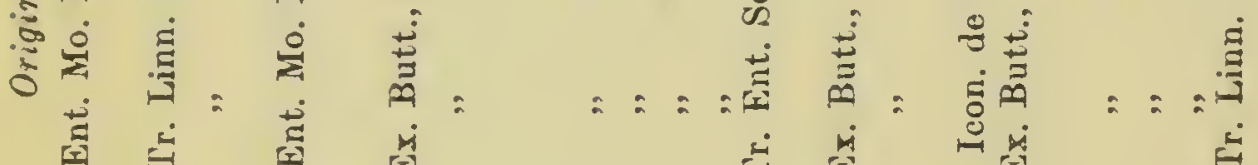

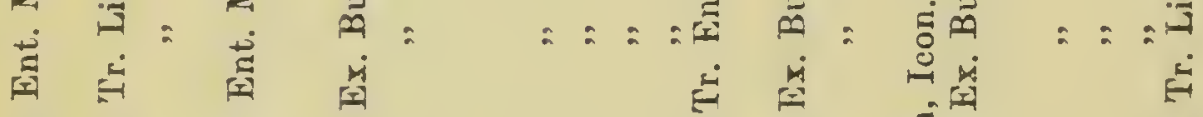

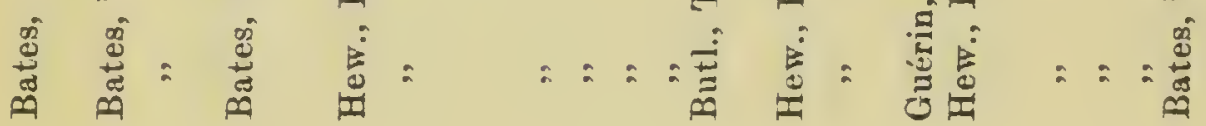

ㅎำ

अं

ชู่ ชั

ริำ

की

รูำ ซิ

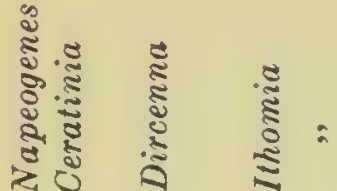

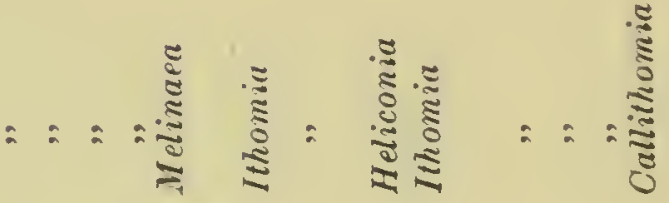

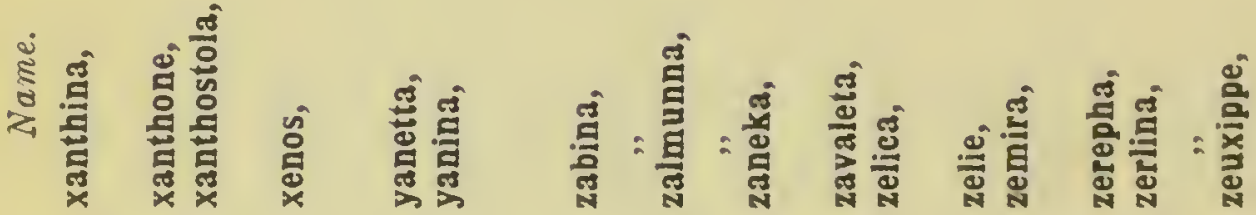

कैले

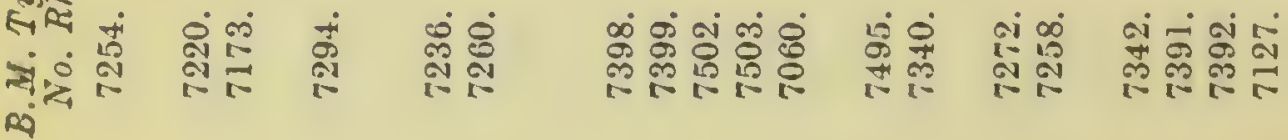




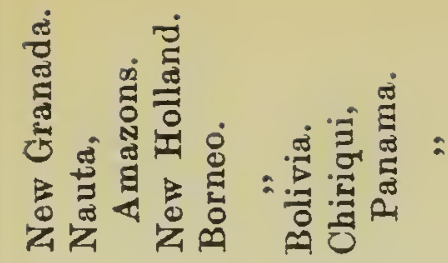

io

Otro ot root ot ro ot

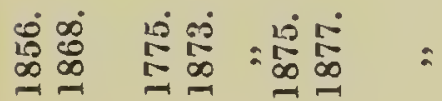

की की की

$=\frac{\vec{R}}{\square} \quad$ वें

คิ

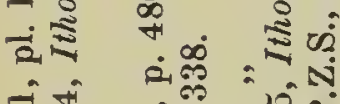

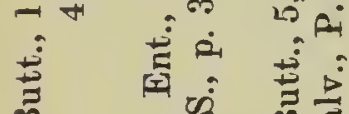

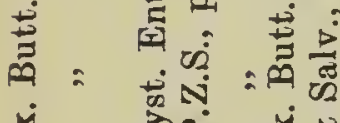

$\Leftrightarrow$ कि

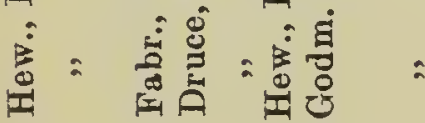

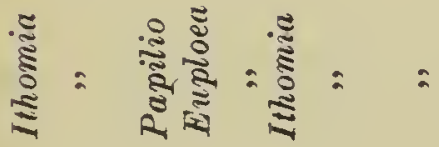

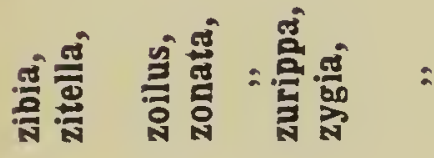

尔 


\section{BRITISH MUSEUM}

\section{(NATLRAL HISTORY) \\ ENTOMOLOGICAL AND GENERAL PUBLICATIONS}

\section{CATALOGUES}

History of the Collections. Vol. I, 15s. Vol. II, £1 10s. Vol. II, Appendix, 58.

Catalogue of the Books, Manuscripts, Maps and Drawings. Vols. I-V, $£ 1$ each. Vol. VI, $£ 215 s$.

Index Animalium, sectio secunda. Pts. I-III, fl. Pt. IV, 15s. Pts. $\mathrm{V}$ and VI, 10s. each.

Report on thc Collections of Natural History made in the Antarctic Regions during the voyage of the Southern Cross, $£ 2$.

National Antarctic Expedition, 1901-4. Vol. I, Geology, fl 10s. Vol. II, Zoology, £3. Vol. III, Zoology and Botany, £2 10s. Vol. IV, Zoology, fl 15s. Vol. V, Zoology and Botany, fl I0s. Vol. VI, Zoology and Botany, $16 s$.

British Antaretic (Terra Nova) Expedition. Natural History Report. Zoology, Vols. I-VIII containing various Parts. Geology, Vol. I, 1-6. Botany, Pts. I, II, III. (Further details on application.)

First Report on Fconomic Zoology. $6 s$.

Sccond Report on Economic Zoology. (is.

Army Biscuit Report. $2 s$.

Nomenclature of Coleopterous Inscets. Pt. VII, 2s. 6d. Pt. VIII, 3s. 6d. (Remaining Parts out of print.)

Illustrations of Typical Specimens of Colcoptera. $16 \varepsilon$.

Catalogue of the Colcopterous Insects of Madeira. $3 s$.

Catalogue of the Coleopterous Insects of the Canarics. 10s. $6 \mathrm{~d}$.

Catalogue of Halticidac. Pt. I, 78 .

Catalogue of Hispidac. Pt. I, $6 s$.

Catalogue of Hymenopterous Insects. P'ts. III-V, 6s. cach. Pt. VII, 2s. (Remaining Parts out of print.)

Revision of the Ichneumonidac. Pt. I, 4s. Pt. II, 5s. 6d. Pt. III, 5s. 6d. Pt. IV, 6s.

Monograph of the Culicidac. Vol. IV, £1 12s.6d. Vol, V, £l 5s. (Iols. $I-I I I$ out of print.)

The Syrphidae of the Ethiopian Region. $6 s$.

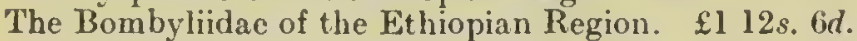

Catalogue of the Lepidoptera Phalacnac. Vol. I, Text, 15s., Plates, 15s. Vol. II, Text, 18s., Plates, 15s. Vol. III, Text, 15s.. Plates, 16s. Vol. IV, Tcxt, 15s., Plates, 16s. Vol. V, Text, 15s., Plates, 15s. Vol. VI, Text, 15s., Plates, 10s. Vol. VII, Text, 17s., Plates, 13s. Vol. VIII, Text, 15s., Plates, 12s. Vol. IX, Text, 15s., Plates, 12s. Vol. X, Text,

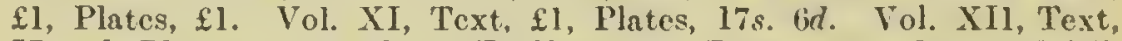
I7s.6d., Plates, $f 15 s$. Vol. XIII, Text, 16s., Plates, 16s. Suppl. Vol. I, Text, fl 5s., Plates, $f 1$ 13s. 6a. Suppl. Vol. II, Text, $£ 1$ 10s., Plater fl $12 \%, 6 d$.

Illustrations of Typical Specimens of Isepidoptera Hetcrocera. Pt. V, $£ 2$ l0s. Pt. VI, £2 4s. Pt. VII, £2. P't. V'III, f2. Pt. IX, £2 as. (Remaining Parts out of print.)

C'atalogue of the Collection of Palaearetic 13utterflies. \&l.

Catalogue of the Type Specimens of Lepidoptera Rhopalocera. Pt. I, 4s. 6d. Pt. II, $4 s .6 d$. 
Catalogue of the Specimens of Neuropterous Insects. Pt. I, 6d.

Catalogue of Orthopterous Insects. Pt. I, £3.

Synonymic Catalogue of Orthoptera. Vol. I, 10s. Vol. Il, 15s. Vol. III, £l.

A Synonymic Catalogue of Homoptera. Pt. I, 5s.

Catalogue of British Hymenoptera. Pt. I, 6s.

Catalogue of British Hymenoptera of the Family Chalcididae, 3s. $6 d$.

Map slowing the known distribution in England and Wales of the Anopheline Mosquitoes. 2s. $6 d$.

Handbook of Britisl Mosquitoes. $£ 1$.

List of the Spccimens of British Animals. Pt. V, Lepidoptera, 2nd Ed. 1s. 9d. Pt. XII, 9d. Pt. XIII, Hymenoptera, 1s. 4d. Pt. XIV, Neuroptera, 6d. Pt. XV, Diptera, 1s. Pt. XVI, Lepidoptera, $3 s$.

\section{GUIDES}

Guide to the Exhibited Serics of Insects. 2s. Postage $3 d$.

\section{INSTRUCTIONS FOR COLLECTORS}

Handbook of Instructions for Collectors. 5s. Postage $3 d$.

No. 4. Insects. 6d. Postage ld.

" 5. Diptera. 6d. Postage ld.

,, 6. Mosquitoes. 6d. Postage $1 d$.

, 7. Bloodsucking Flies, Ticks, \&c. 3d. Postage $1 d$.

\section{ECONOMIC SERIES}

No. 1. The House-Fly as a Danger to Health. 3d. Postage $1 d$.

," 1. The House-Fly. Its Life-history and Practical Measures for its suppression. 1s. 6d. Postage $1 \frac{1}{2} d$.

,2. The Louse as a Menace to Man. 6d. Postage $1 d$.

" 3. Fleas. 4d. Postage $1 d$.

, 4. Mosquitoes. 4d. Postage $1 d$.

" 5. The Bed-bug. 2d. Postage $1 d$.

, 11. Furniture Beetles. 6d. Postage 1d.

, 12. The Cockroach. 6d. Postage ld.

Economic Leaflet. No. 1. The Danger of Disease from Flies and Lice. Pp. 4. $1 d$. for three copies, or $2 s .6 d$. for 100 copies.

Poster Leaflcts, 18 in. by 12 in. :-

The Fly Danger. $\frac{1}{2} d$.

The Mosquito Danger. $1 d$.

The Louse Danger. $\frac{1}{2} d$.

The above publications may be purchased at the Natural History Museum, Cromwell Road, London, S.W.7. Postal applications should be accompanied by a remittance, including a sum to cover postage. 


\section{(NATURAL HISTORY)}

\section{SETS OF PICTURE POSTCARDS}

Set A 1. Views of the Building. No. 1. 10 monochrome cards. 18. A 2. Ditto No. 2. 10 monochrome cards 18 .

B 2. Carnivores. 10 monochrome cards. 18

B 3. Insectivores and Bats. 5 monochrome cards. Bd.

B 4. Rodents. 5 monochrome cards. $6 d$.

B 5. Large Ĺngulates. 10 monochrome cards. 18.

B 6. Monotremes, Marsupials and Edentates. 10 monoclirome cards. $1 s$.

B 7. Antelopes. 10 monochrome cards. 18 .

B 8. British Mammals. 5 monoclrome cards. Gd.

B 9. British Dogs. 5 monochrome cards, 6d.

B 10. British Sporting Dogs. 5 monochrome cards. $b d$

B 11. Dogs : Foreign Breeds. No. 1. 5 monochrome cards. $6 d$.

B 12. Ditto No. 2. 5 ," Gd.

Toy Dogs. 5 monochrome cards. $6 d$.

Birds : British Series. No. 1. 10 monochrome cards. $1 s$. Ditto No.2. 10 ,, 18

Birds : General Series. No. 1. $10 \quad$ ".

$\begin{array}{llll}\mathrm{C} & 4 . & \text { Ditto } & \text { No. } 2.5 \text { coloured cards. '18. } \\ \mathrm{C} & 5 . & \text { Ditto } & 18.5 \\ \mathrm{C} & 6 . & \text { Pigeons, Ducks and Fowls. } 5 \text { monochrome cärds } 18 .\end{array}$

$\begin{array}{llll}\text { C } & 4 . & \text { Ditto No. 2. } 5 \text { colourcd cards. } 18 . \\ \mathrm{C} & 5 . & \text { Ditto } & \text { No. } 3.5 \\ \mathrm{C} & 0 . & \text { Pigeons, Ducks and Fowls. } & \end{array}$

$\begin{array}{llll}\text { C } & 4 . & \text { Ditto No. 2. } 5 \text { colourcd cards. } 18 . \\ \mathrm{C} & 5 . & \text { Ditto } & \text { No. } 3.5 \\ \mathrm{C} & 0 . & \text { Pigeons, Ducks and Fowls. } & \end{array}$

C F-10. Eggs of British Birds. Nos. 1-4. Four sets each of 5 coloured cards. 18. each.

C 11. British Birls: Summer Visitors. No. 1. 5 coloured cards. 18.

C 12. Ditto Winter Visitors. 5 ", ", 18.

C 13. Ditto Summer Vlsitors. No. 2. 5 coloured cäras. 1s.

C 14. Ditto Ditto No. 3. 5 " "1s.

D 1.

1.

D 2 .

Mcteorites. 10 monochroine cards. 18.

Britislı Metcorites. 5 monochrome cards. od.

Representative British Insects. 10 monochrome cards. 18.

E 2. Remarkable Insect Types. 10 monochrome cards. 18.

E 3. Noxious Inscets. 10 monochrome cards. 18.

E 4. Representative Foreign Insects. Series No. 1. 10 monochrone cards. 18.

E 5. British Butterflies. Series No. 1. 5 coloured cards. 18.

E 6. Exotic Butterflies. Series No. 1. 5 coloured cards. 18.

E 7 . British Motlıs. Series No. 1. 5 coloured cards. 18.

F 8. Exotic Moths. Serles No. 1. 5 coloured cards. 18.

I $\cap$. Exotic Butterflies. Series No. 2. 5 coloured cards. 18.

I 10. Ditto No. 3.5 . 18.

E 11. Exotic Moths. Series" No. 2. 5 coloured 'eards. "1s.

F 12. Ditto , No. 3. 5 , , 18 .

E 13. Exotic Beetles. Sèries No. 1. 5 colourcd cäds. 1s.

E 14. Exotic Dragonflies. 5 coloured cards. 18.

E 15. Exotic Neuroptera. 5 coloured cards. 18.

E 16. Fxotic Grassloppers and Praying Insects. 5 coloured cards. $1 s$.

F 17. Exotic Hoinoptera. Series No. 1. 5 coloured cards. 18.

P. 18. Ditto , No. 2. 5 , , 18 .

E 19-24. British Rutternies. N̈o. 2-7. Six sets each of 5 coloured cards. 1s. caclı.

E 25-27. Exotic Moths. Nos, 4-6. Three sets cach of 5 colourcd cards. 1s, each.

E 28. British Moths. No. 2. 5 coloured cards. 18 .

F 1. Remarkable Plant Structures. 5 monochrome cards. Gd.

F 2. Germination of Wheat. 5 monoclrome cards. $6 d$.

, F 3. Dispersal of Fruits and Secds, 20 cards ( 15 in monoclirome and 5 in colour). $28.6 d$.

F 4-9. British Flowering Plants. No. 1-6. Six sets cach of 5 coloured cards. 18. each.

G 1. Fossil Man. 10 monochrome cards. 18 .

(f 2. British Pleistocene Mammals. 10 monochrome cards, $1 s$.

G 3. Fossil Fishes. 10 monochrome cards. 18

f. 4. Fossil Reptiles, 10 monochrome cards. is

A 5. Tertiary Fossil Plants. 10 monoclirome cards. 18

G 6 . Restorations of Fixtinct Mammals. Series No. 1. 10 monochrome cards. 1..

G 7. Restorations of Extinct Reptiles. Series No.1. 10 monochrome cards. 18.

H 1. Rare and Curious Books. No. 1. 10 monochrome cards, 18.

$\mathrm{K}$ 1. Frogs and Toads. 5 coloured cards. 18.

$\mathbf{K}$ 2. Reptiles and Batrachians. No. 2. 5 molioclirome cards. ful.

No.

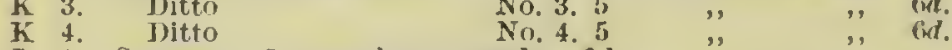

I 1. Sponges. 5 monoclirome cards. $6 d$.

I. 2. Stony Corals. 5 monochrome cards. $B d$.

M 1. Life-Ilistory of the Common Ecl. 5 monochrome cards. om?.

Each set is acconpanied by a descriptive and informative leaflet. individual momo(lirome cards may be obtained separately, price $1 d$; alld iudividual coloured ones, price $2 \pi$. A detailed list will be sent on application.

Orders for the above should be addressed, witl the appropriate remittance, including postage, to -

TIE D)!RECTOR,

IBritislr Museum (Natural 1Iistory). Crounwell Toat, I,ondon, s.,W. 7 .

The postage on one set of five cards is $1 d$, on one set of 10 eards $11, l$, and on one sct of 20 cards 2 th. A hialfucmy should be added for cach additional set of 10 eards and for cvery two additional sets of 5 cards, and a penny for cach additional set of 20 eards. 

$360 \%$

$=$

(3) $=0.090$

(0, 30 \%

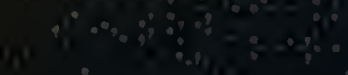

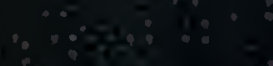

- a. 60 